\title{
Tropical cyclogenesis in a tropical wave critical layer: easterly waves
}

\author{
T. J. Dunkerton ${ }^{1,2}$, M. T. Montgomery ${ }^{2}$, and Z. Wang ${ }^{2}$ \\ ${ }^{1}$ NorthWest Research Associates, Bellevue WA, USA \\ ${ }^{2}$ Naval Postgraduate School, Monterey CA, USA
}

Received: 3 January 2008 - Published in Atmos. Chem. Phys. Discuss.: 9 June 2008

Revised: 22 June 2009 - Accepted: 22 June 2009 - Published: 6 August 2009

\begin{abstract}
The development of tropical depressions within tropical waves over the Atlantic and eastern Pacific is usually preceded by a "surface low along the wave" as if to suggest a hybrid wave-vortex structure in which flow streamlines not only undulate with the waves, but form a closed circulation in the lower troposphere surrounding the low. This structure, equatorward of the easterly jet axis, is identified herein as the familiar critical layer of waves in shear flow, a flow configuration which arguably provides the simplest conceptual framework for tropical cyclogenesis resulting from tropical waves, their interaction with the mean flow, and with diabatic processes associated with deep moist convection. The recirculating Kelvin cat's eye within the critical layer represents a sweet spot for tropical cyclogenesis in which a proto-vortex may form and grow within its parent wave. A common location for storm development is given by the intersection of the wave's critical latitude and trough axis at the center of the cat's eye, with analyzed vorticity centroid nearby. The wave and vortex live together for a time, and initially propagate at approximately the same speed. In most cases this coupled propagation continues for a few days after a tropical depression is identified. For easterly waves, as the name suggests, the propagation is westward. It is shown that in order to visualize optimally the associated Lagrangian motions, one should view the flow streamlines, or stream function, in a frame of reference translating horizontally with the phase propagation of the parent wave. In this co-moving frame, streamlines are approximately equivalent to particle trajectories. The closed circulation is quasi-stationary, and a dividing streamline separates air within the cat's eye from air outside. The critical layer equatorward of the easterly jet axis is important to tropical cyclogenesis because its cat's eye provides (i) a region of cyclonic vorticity and weak deformation by
\end{abstract}

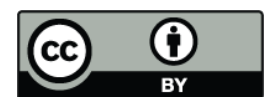

Correspondence to: T. J. Dunkerton (tim@nwra.com) the resolved flow, (ii) containment of moisture entrained by the developing gyre and/or lofted by deep convection therein, (iii) confinement of mesoscale vortex aggregation, (iv) a predominantly convective type of heating profile, and (v) maintenance or enhancement of the parent wave until the vortex becomes a self-sustaining entity and emerges from the wave as a tropical depression. The entire sequence is likened to the development of a marsupial infant in its mother's pouch. These ideas are formulated in three new hypotheses describing the flow kinematics and dynamics, moist thermodynamics and wave/vortex interactions comprising the "marsupial paradigm". A survey of 55 named tropical storms in 19982001 reveals that actual critical layers sometimes resemble the ideal east-west train of cat's eyes, but are usually less regular, with one or more recirculation regions in the co-moving frame. It is shown that the kinematics of isolated protovortices carried by the wave also can be visualized in a frame of reference translating at or near the phase speed of the parent wave. The proper translation speeds for wave and vortex may vary with height owing to vertical shear and wave-vortex interaction. Some implications for entrainment/containment of vorticity and moisture in the cat's eye are discussed from this perspective, based on the observational survey.

\section{Introduction}

The genesis of tropical cyclones, hurricanes and typhoons is one of the most important unsolved problems in dynamical meteorology (Emanuel, 2005) and climate (Gore, 2006). As for why the problem remains unsolved, after decades of research, it is unfortunately true that in situ observations of genesis are mostly lacking over remote tropical oceans, that field campaigns are too few while operational efforts generally target mature storms, that critically important processes and their multi-scale interactions are challenging to model and observe. Nature in some cases provides little advance

Published by Copernicus Publications on behalf of the European Geosciences Union. 
warning of these storms and prediction of genesis beyond $48 \mathrm{~h}$ is generally too uncertain to be useful. Funding and technological resources are needed to remedy these deficiencies, to the extent they can be remedied, but - to be honest - it is unlikely that fundamental progress will be made without a quantum leap in theoretical understanding as well. As will be demonstrated in this paper and the sequels, available observations on the synoptic scale need to be analyzed in a manner that is consistent with the Lagrangian nature of tropical cyclogenesis. As a moist diabatic vortex, the tropical cyclone, through its lifecycle from genesis to intensification, represents a coherent interaction between potential vorticity (PV) and moist entropy, both of which are adiabatic invariants, i.e., material properties of a conservative flow. In the earliest stage of genesis, the fluid motion is mostly horizontal and quasi-conservative, punctuated by intermittent deep convection, a strongly diabatic and turbulent process. In order to fully appreciate the transport of PV and moist entropy by the flow, their interaction with one another, the impacts of deep convective transport and protection of the proto-vortex from hostile influences requires, among other things, an understanding of material surfaces or "Lagrangian boundaries" in the horizontal plane. This viewpoint, although used subconsciously by forecasters ${ }^{1}$, is invisible to researchers working with standard meteorological products in an Eulerian or Earth-relative framework. For tropical cyclogenesis it is nec-

\footnotetext{
${ }^{1}$ The elusive but sometimes exposed "low cloud swirls" and other stratiform cloud or water vapor anomalies represent $L a$ grangian entities to the extent that they are passively advected by the flow. Feature-tracking provides instantaneous Eulerian winds (e.g., Velden et al., 1997, 2005), while animation of the accumulated horizontal displacement of cloud or water vapor anomalies (away from their sources and sinks: viz., deep convection and other regions of moist ascent) effectively provides a Lagrangian view. So the geostationary imagery are helpful in this respect, whereas standard meteorological charts in the Earth-relative frame and infrequent snapshots from polar orbiting satellites are not. Surface winds from scatterometry are indisputably valuable, but it is unnecessary for such images (as from QuikSCAT or ASCAT) to display a closed circulation in order for a closed circulation to exist in a Lagrangian sense (see Appendix D). A morphed animation of total precipitable water from microwave imagery (beginning in 2006) is proving very helpful for analysis and prediction of moist tropical waves in the lower troposphere (http://cimss.ssec.wisc.edu/tropic2). Forecasters are now using this product to detect, locate and extrapolate the position of wave troughs. By viewing the sequence of frames, the viewer obtains a sense of Lagrangian horizontal motions weighted by the vertical profile of moisture, together with the impacts of convective moistening and horizontal entrainment. Extraction of quantitative Lagrangian information from sequential imagery is far from trivial, but a worthwhile goal. When it comes to closed circulations in propagating waves, the difference between Eulerian and Lagrangian flow is critically important, as will become clear in this paper. Forecasters deal simultaneously with both viewpoints in their arsenal of observations, yet (to our knowledge) without a clear differentiation between them.
}

essary to understand the morphology of Lagrangian transport, and it is desirable to know whether, and how, suitable information for this purpose can be obtained from an intelligent manipulation of Eulerian data.

Compounding the problem is that multiple pathways to genesis exist. Four plausible pathways identify one or more of the following as key ingredients: (i) tropical waves, (ii) monsoon troughs, (iii) extratropical disturbances and (iv) topographic flows ${ }^{2}$. For each pathway we can imagine a theoretical paradigm that provides conceptual stepping stones along the path. In tropical waves, a sweet spot for development evidently exists along the wave, associated with a region of low pressure in the lower troposphere. Having such a sweet spot does not guarantee genesis, of course, because other environmental factors also play an important role in deciding whether or not a storm may form. Many individual wave-troughs occur in a single hurricane season but only a fraction - approximately $20 \%$ in the eastern and central Atlantic $^{3}$ (Frank, 1970) - contribute to the formation of a tropical depression-strength vortex (defined hereafter as "genesis"). Among those disturbances that produce depressions almost all have a "surface low along the wave" in the forecaster's familiar parlance. Few are thought to have a midlevel cyclonic vortex without the surface signature, a negligible fraction of the original wave count. Mid-level vortices without a discernable surface circulation are indeed observed in the tropics, increasingly so as satellite remote sensing improves (e.g., shortwave IR) in regions of frequent deep convection, such as the ITCZ. Some have suggested that tropical mesoscale convective systems produce mesoscale convective

\footnotetext{
${ }^{2}$ The third and fourth pathways do not represent tropical cyclogenesis genesis in the pure sense of the term. The third pathway is not entirely tropical, and the fourth pathway is not entirely oceanic. Therefore if one is interested in pure TC genesis, the problem reduces to (i) a tropical-wave pathway and (ii) a geophysical turbulence pathway. The latter may be subdivided into quasi-2-D and rotating 3-D-convective pathways to highlight the role of horizontal circulations and deep moist convection in a rotating environment, respectively. One can then appreciate the fuzzy nature of the classification scheme, as waves and turbulence generally coexist (Lilly, 1983; McWilliams, 1984; McIntyre, 2003). Our subdivision of pathways becomes counter-productive if applied too rigidly! Genesis is not a question about which of these mechanisms is acting in a particular situation, but rather, how much of each, and how they interact across disparate scales.

${ }^{3}$ The percentage estimate is non-trivial: viz., to evaluate the percentage of parent African easterly waves that generate named or unnamed storms anywhere in the Atlantic sector. It's not a question of where TDs were born; rather, about where their precursor waves, if any, came from. A typical season has 60 waves and 17 named or unnamed storms. This yields an upper limit of $28 \%$ for the average, noting that in some years the percentage may be much smaller, or in few years, much larger (e.g., 2005). Arbitrarily allowing 5 storms from non-AEW (e.g., baroclinic) sources brings the average count down to 12 , or $20 \%$. This number matches Frank's (1970) derived from 3 seasons.
} 
vortices initially without a cyclonic circulation near the surface (Bister and Emanuel, 1997; Ritchie and Holland, 1997) and that this may be a reason why only a small fraction of disturbances become tropical depressions (E. Zipser, personal communication; Harr and Elsberry, 1996). It will be shown here that for storms originating in tropical waves, the synoptic wave itself plays a vital role in the near-surface circulation. In our proposed tropical-wave scenario the relevant theoretical paradigm is the formation of a closed proto-vortex within the wave in the lower troposphere and subsequent eddy shedding whereby the vortex, energized further by deep convective heating, becomes detached from the wave and begins to control its own destiny. In what follows we refer to this sequence of events as the marsupial paradigm. Central to our presentation of the marsupial paradigm is a rudimentary understanding of the Lagrangian flow and its dynamical and thermodynamical properties in the neighborhood of the developing storm.

Our series on TC genesis, of which this paper is the first, addresses the tropical wave pathway and variations thereof (easterly wave propagation, tropical depression disturbances, instability of the ITCZ, and trapped equatorial modes) and the ability of these disturbances to create a closed gyre or "pouch" and subsequently within the pouch, a proto-vortex or "embryo" favorable for hurricane formation ${ }^{4}$. We are concerned also with the monsoon trough pathway (e.g., Harr and Elsberry, 1996; Simpson et al., 1997). But for TC genesis, environmental conditions in the monsoon trough (McBride and Zehr, 1981) appear favorable over a larger area, providing a diverse set of flow configurations in the lower troposphere (shear line, confluence, gyre; Ritchie and Holland, 1999) and making the role of tropical waves (with their sweet spot) more challenging to ascertain - although precursor waves can be seen in many of these cases. Genesis by spontaneous aggregation (now a popular subject of research: e.g., Emanuel and Nolan, 2004; Nolan et al., 2006; Held, 2007; Borth, 2007; Schecter and Dunkerton, 2009) may be

\footnotetext{
4The "embryo" is borrowed from other authors (Emanuel, 1993, 2007; M06) so to be consistent with the marsupial paradigm we might refer to the proto-vortex as a "joey". Extending the analogy, a female joey provides a second "baby pouch" for convective organization at smaller scales. Little is known about the hierarchy of clouds and vortices in the pouch, and how they interact, but such details are crucial for understanding. Observations sometimes suggest multiple low-level circulation centers in systems that are still becoming organized. Forecasters and scientists alike want to know more about these proto-structures; e.g., their role in Ekman pumping and convective triggering. For now and the foreseeable future, the LT in developing environments remains a mysterious realm often obscured by patches of deep convective cloud and stratiform anvils. Exposed low-cloud swirls are marvelous to behold, but they were likely hidden by deeper cloud when generated, and will be hidden again when new convection erupts. Geostationary microwave technology, needed to penetrate this environment continuously, is $\sim 20$ years away.
}

regarded as a limiting case of the monsoon trough pathway in which no particular region is favored, but conditions are rendered more favorable, e.g., by cranking up the underlying SST. In the real world, spontaneous aggregation is likely nature's last resort ${ }^{5}$. En route to storm formation, the tropical atmosphere almost always provides a sweet spot or more extensive region favorable to genesis. We believe this fact has important implications for the study of tropical cyclogenesis in climate change. Whereas an increase of SST is expected naively to increase the intensity of mature tropical cyclones, the effects of climate change on tropical cyclogenesis will be felt more immediately by modulation of local environments that are already favorable for genesis in the current climate; effects that will be manifested, for instance, in a changed morphology of tropical waves, monsoon troughs and their favorable regions, as well as changes of large-scale circulation and ITCZ that affect storm tracks and environment (Frank and Young, 2007; Vecchi and Soden, 2007; Ventham and Wang, 2007; Vimont and Kossin, 2007; Wing et al., 2007) possibly directing storms into regions of warmer $\mathrm{SST}^{6}$.

In the study by Montgomery et al. (2006b; hereafter M06) the TC genesis problem was posed as the metamorphosis of a mid-level mesoscale convective vortex (MCV) with weak cyclonic circulation at the ocean surface into a self sustaining warm-core tropical depression vortex. The initial MCV embryo was assumed to be a by-product (e.g., Emanuel, 1993; Bister and Emanuel, 1997; Raymond et al., 1998) of one or more mesoscale convective systems (MCSs) that form and evolve within the trough region of an African easterly wave in the Atlantic basin, or monsoon trough in the western Pacific basin. The problem was examined using a non-hydrostatic full physics cloud model (RAMS) at 2 $\mathrm{km}$ and $3 \mathrm{~km}$ horizontal grid spacing for realistic sea surface temperatures (SSTs) and thermodynamic vertical profiles of moist static energy within the low pressure region of

\footnotetext{
${ }^{5}$ In the "real world" of 3-km simulations performed on Earth Simulator (Matsuno 2006, 2007) tropical easterly waves play a crucial role in TC genesis, for present-day climate conditions.

${ }^{6}$ Genesis depends on the spatial pattern as well as the amplitude of the underlying SST, and most SST variations are contained in coupled patterns of atmosphere-ocean variability that oscillate with time. Even if one could somehow isolate a simple-pattern/seculartrend of SST variation and study its effect on the general circulation (as done by Magnusdottir, 2001 for the NH winter season) the effect on genesis is non-trivial. Consider the following: a horizontally uniform change in underlying SST, e.g., the simplest of all changes in an altered world, would be superposed on the existing distribution that is a strong function of latitude. This distribution is responsible, inter alia, for the latitude of the ITCZ. The impact of a uniform change is therefore also a function of latitude owing to the strong nonlinearity of the Clausius-Clapeyron relation. With changes in SST and ITCZ come changes in the tropical waves and instabilities (hence, hurricanes) that depend, directly or indirectly, on the structure and stability of circulations associated with the ITCZ. Such changes impact the hydrological cycle on a global scale, with regional consequences (IPCC, 2007).
} 
a tropical disturbance. M06 obtained new insight on convective and mesoscale processes operating within such an idealized pre-genesis environment. In the absence of hostile influences, such as strong vertical wind shear or dry air intrusions, M06's high-resolution simulations demonstrated an upscale cloud/mesoscale mechanism for building the incipient tropical storm vortex. This mechanism was shown to operate on realistic time scales and, equally important, to precede the wind-speed/evaporation feedback believed to govern the intensification of an already formed tropical depression strength surface vortex (Rotunno and Emanuel, 1987; Emanuel, 1989).

Within the convectively unstable and cyclonic-vorticity rich environment of the initial MCV embryo, horizontally small-scale warm-core updrafts possessing intense cyclonic vorticity in their cores ("vortical hot towers"; VHTs) emerged spontaneously as the dominant coherent structures. As often observed in real developing storms, the most prominent VHTs were triggered at a finite radius from the protocirculation center, consistent with the expected radial dependence of Ekman-like pumping in a geophysical vortex boundary layer (Smith, 1968; Eliassen and Lystad, 1977). Concurrent with this low-level vortex spin-up process are localized downdrafts and spreading gust front boundaries. Minima in equivalent potential temperature occur in association with localized downdrafts that import low equivalent potential temperature from the middle troposphere into the surface boundary layer. Unlike warm season continental or maritime mesoscale convection that often favors linear propagating structures (squall lines), the spreading gust front boundaries were confined laterally, to some extent, by the near-surface cyclonic circulation and the low-level inflow generated by the mean latent heating in the vortex towers. M06 showed that this low level inflow was reasonably well captured by the axisymmetric Eliassen balanced circulation (Shapiro and Willoughby, 1982; Wirth and Dunkerton, 2006) driven primarily by the mean (temporally and azimuthally averaged) heating rate of the vortex towers. The rotational confinement of these gust front boundaries appears important for sustained deep convective activity within the MCV embryo and surface spin-up. Even when gravity waves and gust fronts are not fully contained, they can be expected to play an important role in convective triggering along developing vortical boundaries in the proto-storm.

The numerical experiments of M06 demonstrated a plausible vortex assembly process that can occur within a favorable environment possessing a small but finite cyclonic surface circulation,. Yet to be answered, however, is how this "embryo" and its surrounding circulation are created in the first place and whether the initial proto-vortex can survive long enough in realistic environments (e.g., with vertical shear or dry air intrusions) to enable the transition to a self-sustaining warm-core vortex. The numerical problem, as posed by M06 and others, specifies environmental conditions and a seedling disturbance according to their observed rela- tionship (Gray, 1998) but explains neither of them, nor their association. What is special about a tropical wave trough? What latitude along the trough axis is favorable for storm development? What characteristics of a monsoon trough are important for genesis? Where do storms form in relation to the monsoon trough, and why? These questions transcend the local processes considered by M06, and require a connection to be drawn between the proto-vortex environment and synoptic-scale flow. Because of the strongly helical nature of the VHTs, another aspect not yet investigated concerns the role of helicity (Moffat, 1969) in stabilizing the upscale cascade (Lilly, 1986a,b; Herring and McWilliams, 1989; Weisman and Rotunno, 2000; Kurgansky, 2006), a process that occurs generically within the MCV, parent synoptic wave, or monsoon trough. A major objective of our research is to explain the generation and maintenance of the hurricane embryo within its parent synoptic-scale wave or monsoon trough environment. Another is to advance beyond M06 and understand more completely the role of moist convective (helical) turbulence that operates within the embryo.

The tropical wave pathway to genesis involves

1. the familiar easterly waves of the Atlantic (e.g., Carlson, 1969; Burpee, 1974; Reed et al., 1977; Shapiro, 1977, 1980; Thorncroft and Hodges, 2001) and Pacific basins (Chang et al., 1970; Molinari et al., 1997; Raymond et al., 1998; Molinari and Vallaro, 2000; Molinari et al., 2000);

2. the less familiar (mixed) Rossby-gravity waves of the central and far eastern Pacific Oceans (Hendon and Liebmann, 1991; Dunkerton, 1993; Dunkerton and Baldwin, 1995; Dickinson and Molinari, 2002; Molinari 2004); and

3. the so-called "tropical depression" disturbances of the western tropical Pacific (Liebmann and Hendon, 1990; Lau and Lau, 1990; Takayabu and Nitta, 1993; Dunkerton, 1993; Dunkerton and Baldwin, 1995; Wheeler and Kiladis, 1999; Sobel and Bretherton, 1999; Wheeler et al., 2000).

These wave types comprise a range of ground-based periods from 2.5-9 days. Slower waves such as moist Kelvin waves (Dunkerton and Crum, 1995; Wheeler et al., 2000), equatorial Rossby waves (Molinari et al., 2006) and MaddenJulian oscillation (Maloney and Hartmann, 2000) also modulate tropical waves and tropical cyclogenesis (Maloney and Dickinson, 2003; Aiyyer and Molinari, 2003, 2008). Tropical waves in the 2.5-9 day band may originate from convective coupling (Hayashi, 1970; Lindzen, 1974), lateral forcing (Wilson and Mak, 1984; Randel, 1992; Zhang and Webster, 1992), hydrodynamic instability of the ITCZ (Hack et al., 1989; Schubert et al., 1991; Wang and Magnusdottir, 2005, 2006) or fully developed TCs and their associated "Rossby wave wakes" (McWilliams and Flierl, 1979; Montgomery et 
al., 1999; Ritchie and Holland, 1999). The role of equatorially trapped waves in tropical cyclogenesis requires knowledge of their latitudinal structure and cross-equatorial symmetry, while that of TD disturbances requires that we account for a significant meridional component of propagation. These wave types are examined separately. Excluded from consideration in our first three papers are

4. tropical storms originating from extratropical upperlevel PV anomalies in a moist baroclinic environment (Montgomery and Farrell, 1993) and/or stalled frontal zones in the subtropics (the so-called "tropical transition" pathway: e.g., Davis and Bosart, 2004) and topographic effects (Zehnder et al., 1999; Chang et al., 2003).

Neither of these pathways (TT or topography) are purely tropical and oceanic. A key ingredient of TC genesis - formation of a closed recirculation region - pertains, nonetheless, to many examples of subtropical development in the central and western Pacific that we have encountered in our preliminary survey. A fourth paper will examine these cases.

Local instability of the ITCZ as a pathway to genesis is possible, although more commonly, the waves produced thereby propagate downstream into regions more favorable to genesis: e.g., with warmer water or larger absolute vorticity. Storms generally do not originate within the locus of ITCZ convection, but cause poleward distortion or obliteration of the ambient ITCZ (Dunkerton, 2006). Significant storms in the historical record affecting coastal regions of the southeastern US and Gulf states originated from tropical waves in the eastern and central Atlantic (the "main development region" or MDR) notwithstanding a few spectacular storms originating in the western Atlantic and the Gulf of Mexico. Owing to their quasi-monochromatic nature and relative simplicity, we begin our genesis study in the context of Atlantic easterly waves (Kiladis et al., 2006; Hall et al., 2006) and their eastern Pacific extension. The papers to follow will extend this line of research to other types of tropical waves and instabilities relevant to other ocean basins (Bessafi and Wheeler, 2006; Frank and Roundy, 2006; Molinari et al., 2006). To isolate key elements of the various sub-types of tropical-wave cyclogenesis cited above, we begin our discussion in Sect. 2 with a conceptually simple scenario in which hydrodynamically neutral waves emerge from an unstable source region upstream, propagate westward into the domain of interest, and create regions favorable for genesis; regions which may, in turn, maintain or enhance the neutral waves via convective heating against dissipation while propagating across the basin. This scenario is most relevant to the Atlantic sector, wherein easterly waves generated by moist hydrodynamic instability over Africa (that is, over land, where TCs cannot form owing to surface friction and absence of sustained latent heat fluxes) or by other means propagate westward into a warm ocean, providing seedlings for possible genesis. It is also relevant to other basins, such

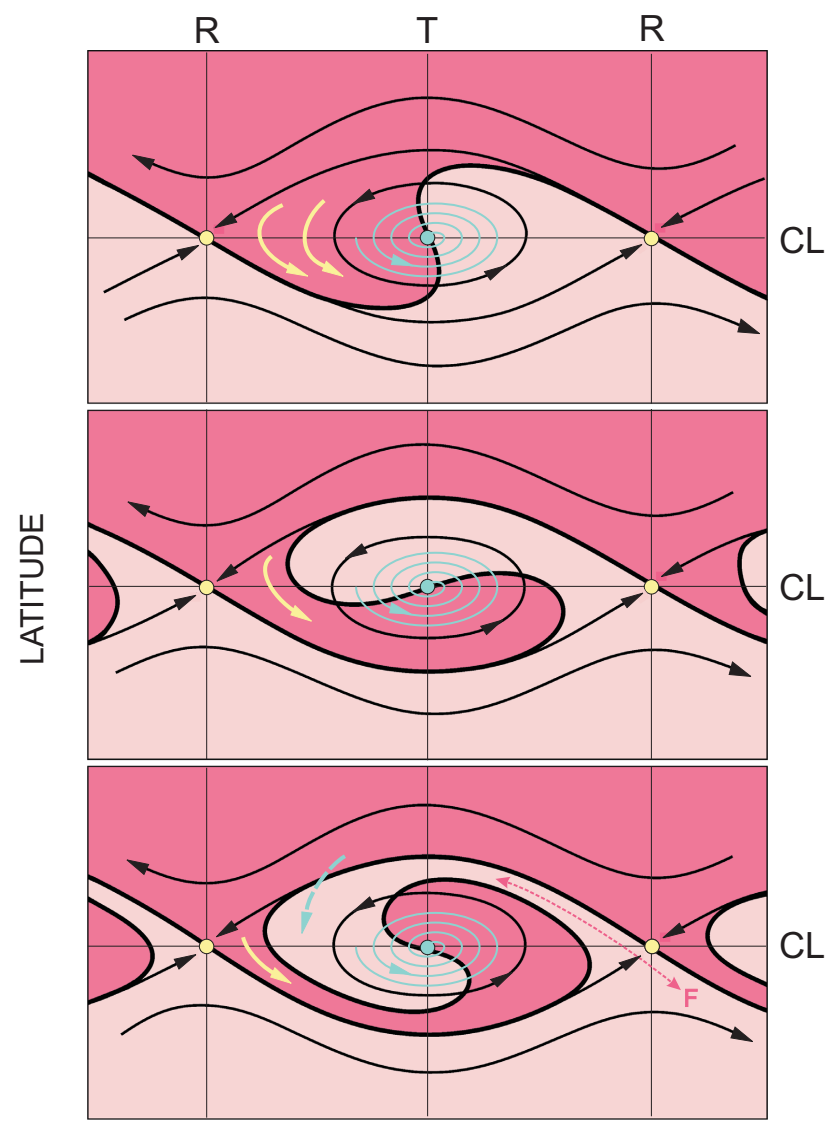

LONGITUDE

Fig. 1. Critical layer for waves in latitudinal shear, showing streamlines (solid), bounding streamline (thick solid) and Trough/Ridge axes (thin solid). If the deeper shade of pink represents higher absolute vorticity, the wave acts on a stable meridional gradient of zonal mean PV, with initial vorticity maximum poleward of the critical latitude (CL). In an unstable configuration, with higher vorticity represented by the lighter shade, the first cycle of overturning entrains higher vorticity from equatorward of the CL axis. The latitudinal shear is negative in either case. Adapted from Andrews et al. (1987).

as the eastern tropical Pacific or mid-Atlantic, but we recognize that in such regions (over ocean, where surface friction is relatively small and latent heat is plentiful), instability of the ITCZ may sometimes lead directly to genesis (Hack et al., 1989; Schubert et al., 1991; Wang and Magnusdottir, 2005, 2006). Some of the events occurring in our time period of interest may belong to this category; they are more akin to instability than the neutral wave propagation imagined in our ideal scenario. Hydrodynamically unstable waves have distinct topological characteristics for a time, but once reaching finite amplitude they are equally good candidates for genesis, occurring within a critical layer, as neutral waves.

What follows is primarily an observational study preceded by formulation and presentation of three new hypotheses 
for tropical cyclogenesis in tropical waves. Three independent datasets are used for meteorological analysis (ERA-40), satellite-derived precipitation (TRMM) and storm history (NHC best-track data). Section 2 outlines the basic theoretical concepts underlying our analysis. Section 3 illustrates the morphology of waves and convection in relation to the mean flow and embedded critical layers, in two representative cases, and summarizes key properties of all storms examined, while Sect. 4 affords a closer look at the 3-D structure and evolution of the critical layer, closed material contours, and diabatic vortices in one of these cases. Details of our analysis method, additional cases, streamline topology and the view from scatterometry are discussed in Appendices A$\mathrm{D}$, respectively. Two contemporary viewpoints of TC genesis and climate change, contrasting the roles of off-equatorial circulation and moisture supply, are noted in Appendix E. The time period of interest encompasses the peak hurricane seasons of 1998-2001 when the two primary datasets used in our study (ERA-40 and TRMM) overlap.

\section{Conceptual fundamentals of the marsupial paradigm}

Whether hydrodynamic instability plays an immediate role in $\mathrm{TC}$ genesis (as sometimes observed in the eastern Pacific and mid-Atlantic) or excites waves that later become instrumental in genesis downstream (as in the African/Atlantic sector) there is a fluid dynamical situation arising in both scenarios that is relevant to genesis for reasons to be enumerated below. This situation involves the familiar critical layer of waves in shear flow, wherein parcels experience irreversible displacements and trapping about the wave's critical level, viz., the location where mean flow and wave phase speeds are equal ${ }^{7}$. In the so-called "cat's-eye" of the critical layer where trapping of parcels takes place, the fluid becomes isolated from its surroundings on either side of the critical layer as parcels outside the cat's eye are advected in opposite directions. The Lagrangian mean flow is asymptotically discontinuous on the edges of the critical layer and its horizontal profile consists of three components: two are in opposite directions on either side, while the middle is stationary with respect to the cat's-eye pattern (Andrews and McIntyre, 1978). The latter is equivalent to a statement that the Lagrangian mean flow in the critical layer is zero in a frame of reference moving with the critical layer itself: i.e., at the phase speed of the wave ${ }^{8}$. Here we take the Lagrangian mean to be a spatial average in the direction of the Eulerian mean flow, which we also as-

\footnotetext{
${ }^{7}$ The critical level is generally a surface in three dimensions, but the word level or line has been used in the literature to represent any of these dimensions, e.g., latitude or altitude.

${ }^{8}$ In latitudinal shear, the cat's eye of the critical layer is uniquely where a wave-centric view is Lagrangian, in the sense described in our response to an Interactive Comment. In the marsupial paradigm, a storm-centric view is approximately equivalent to a wave-centric view, for as long as proto-storm and parent wave move together.
}

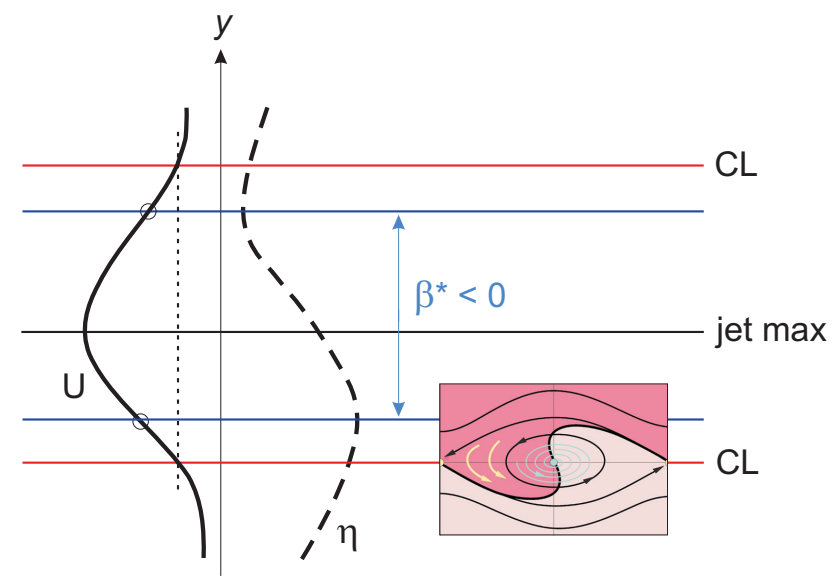

Fig. 2. Idealized profile of an easterly jet $U$ north of the equator, on which stable easterly waves propagate westward having their critical latitudes just outside the jet's inflection points where the gradient of absolute vorticity $\eta$ or "effective $\beta$ " is zero. Unstable waves may be imagined to have their critical latitudes just inside the latitude of inflection points.

sume to be the direction of wave propagation, as shown in Fig. 1. This simplifying assumption will motivate our discussion of the role of the critical layer in TC genesis, but it should be borne in mind we are always concerned with the actual direction of wave propagation in relation to the mean flow, regardless of their azimuthal directions, and will define the critical level generally in terms of the locus of points where the wave intrinsic frequency goes to zero.

\subsection{An idealized easterly jet}

To fix ideas, consider a zonally uniform easterly jet at tropical latitudes off the equator. For simplicity, assume a barotropic jet structure as depicted in Fig. 2. There is cyclonic shear equatorward of the easterly jet maximum, and anticyclonic shear poleward of the maximum. Equatorward of the jet core the cyclonic shear vorticity is enhanced by the planetary vorticity, while poleward of the jet core, the anticyclonic shear vorticity and the planetary vorticity tend to compensate. The absolute vorticity poleward of the jet core is therefore weakly negative or close to zero. Since the meridional gradient of the absolute vorticity changes sign both poleward and equatorward of the jet core, the mean flow satisfies one of the necessary (Rayleigh) conditions for barotropic instability. It has been long recognized in the literature that the African easterly jet (AEJ) is unstable to disturbances which might be described as a moist barotropic instability or mixed barotropic/baroclinic instability ${ }^{9}$. More

\footnotetext{
${ }^{9}$ The critical level plays a central role in such instabilities, enabling phase-locking of counter-propagating Rossby waves in adjacent regions of opposite meridional PV gradient (Hoskins et al., 1985; Heifetz et al., 1999) or, in linear ray theory, as the locus
} 
recently it has been suggested that synoptic-scale instability by itself may be too slow to explain easterly wave disturbances in the African sector (Hall et al., 2006). In either event, if we assume that the locus of wave instability or excitation lies upstream, i.e., that the region of interest is stable with respect to exponential growth, it may be inferred that the phase speed of waves generated upstream lies outside the range of mean flow speeds in the region of reversed PV gradient, as depicted in Fig. 2. The alternative - a critical level within the zone of reversed gradient - would likely cause unstable growth. For an easterly jet north of the equator, the critical level (hereafter, critical "latitude") therefore resides a bit south of the southernmost latitude where the effective beta is zero.

A second critical latitude exists north of the jet axis, north of the northernmost latitude where the effective beta is zero again, on the opposite side of the jet. As already noted, however, the shear vorticity of the mean flow is cyclonic south of the jet and anticyclonic to the north. If a critical layer of finite width were to form at the location of either critical latitude as a result of wave propagation into the region of interest, the sense of rotation in the cat's eye would be the same as that of the mean relative vorticity. For TC genesis we are obviously interested in cyclonic rather than anticyclonic vorticity in the lower troposphere and therefore regard the southern, not northern, critical layer as a possible locus of TC formation. Moreover, we should not imagine that actual wave disturbances and the critical layers they generate are exactly symmetric or antisymmetric about the jet axis ${ }^{10}$. For the case of an idealized unstable easterly jet, Thorncroft and Hoskins (1994a) showed that the most unstable wave disturbance south of the jet is centered equatorward of the southernmost latitude where the effective beta changes sign. Their maximum amplitude is near the critical latitude, in accord with our propagating-wave scenario (as might be expected from consideration of linear parcel displacements, which maximize at the critical latitude or level). This asymmetry of wave amplitude about the axis of the jet, favoring the southern or equatorward side, also emphasizes the cyclonic critical layer at the expense of the anticyclonic one. For the case of an exponentially stable jet flow, discrete neutral or slowly decaying "quasi-mode" wave packets (Reasor

of wave overreflection (Lindzen and Tung, 1978; Lindzen et al., 1983). Either of these mechanisms leads to wave growth if certain conditions are met. In barotropic instability, one of these conditions is the existence of a critical level within the zone of reversed PV gradient. The latter requirement is not universal, however. When $\beta=0$ the "varicose mode" for a symmetric jet has a critical level at the inflection point. More generally, when the interior PV is everywhere uniform, as in the popular Eady model, the reversal of interior PV gradient is moot.

${ }^{10}$ North-south symmetry of the basic state about the jet axis is broken by mean absolute vorticity, which is asymmetric, and a meridional gradient of moisture, if one exists. Further asymmetry may arise from wave sources upstream. and Montgomery, 2001; Schecter et al., 2002) - stimulated, perhaps, by mesoscale processes within the trough region of the wave envelope (Berry and Thorncroft, 2005; Hall et al., 2006) - should possess similar phase speed and wave maxima characteristics as their unstable counterparts. Our immediate concern is not with wave sources, which lie outside the scope of this paper, but with downstream propagation of neutral waves on an easterly jet as depicted in Fig. 2.

\subsection{Three new hypotheses regarding the wave-vortex hybrid}

The formation of a tropical depression in a tropical wave critical layer is plausible only in a cyclonic Kelvin cat's eye, not an anticyclonic one. Therefore we selected the southern, not northern, critical layer as the locus of interest. Formation of a tropical cyclone also requires convective heating in order to achieve the desired levels of potential vorticity and concentration of PV substance: $\sim 100$ times ambient values, in a mature storm. In the ideal critical layer of Fig. 1, PV anomalies within the cat's eye are symmetrically distributed (i) in physical space about the centerline and (ii) in a statistical distribution about the mean value, which is cyclonic here, because we have chosen the southern CL on the AEJ. Passive roll-up of cyclonic mean vorticity in the southern critical layer does not lead directly to genesis, because local extrema of PV anomalies are preserved by a dry adiabatic rearrangement, in ideal and real-life wave scenarios. It does so indirectly, in the sense that this "coherent structure" of cyclonic vorticity (created at meso- $\alpha$ scale by a synoptic ${ }^{11}$ wave) provides one or more seedlings for amplification by mesoscale convective processes (M06) which, as we now argue, might occur preferentially in the critical layer. As shown in Fig. 1, the longitudinal center of the cat's eye corresponds to the trough of the wave, a region favorable for deep moist convection in observations. (i) Formation of a Kelvin cat's eye by critical layer development at meso- $\alpha$ or synoptic scales, and (ii) convective enhancement and up-scale aggregation of cyclonic vorticity from meso- $\gamma$ to meso- $\beta$ scales, is a plausible outcome of the neutral wave propagation depicted in Fig. 2. The order of enumeration is important: the cat's eye is a result of wave propagation into the region of interest, while convective amplification and aggregation is aided subsequently by formation of the cat's eye. Causality is determined, in part, by the arrow of time: what follows temporally (item ii) cannot be a cause of what came before (item i). This idea was expressed clearly in theories of the ideal dry Rossby-wave critical layer (Killworth and McIntyre, 1985, and references therein) wherein the cat's eye precedes (logically and chronologically) the redistribution of PV within,

\footnotetext{
${ }^{11}$ Following convention, $2.5-9$ day moist tropical waves relevant to TC genesis occur on synoptic spatial scales (2000-8000 km or so) while cat's eyes and closed recirculation regions develop at meso- $\alpha$ $(200-2000 \mathrm{~km})$. Proto-vortex development occurs at meso- $\beta$ (20$200 \mathrm{~km})$ involving deep moist convection at meso- $\gamma(2-20 \mathrm{~km})$.
} 
and the advecting flow does not depend to leading order on the redistribution itself. The reader may consult our Final Response at the ACPD website for further discussion, citations and quotations regarding this point. These considerations do not exclude the possibility that upscale mechanisms may have created the easterly wave in the first place (as seen over the African Highlands) or that new Rossby waves may be excited in the wake of a mature storm (as seen in western Pacific TD disturbances) as noted in the Introduction. Nor do they preclude thermal forcing of the parent wave by the developing proto-vortex within. The proper sequence is appreciated most clearly in our neutral wave scenario on a parallel shear flow, without the clutter introduced by secondary phenomena.

Important to this sequence of events is that the easterly jet is located in the lower, not middle, troposphere and that lower tropospheric vorticity is organized by the Kelvin cat's eye in this layer, and subsequently amplified by convective processes in the cat's eye. In real life the waves and their effects are more complicated than in our ideal scenario; actual waves are local, episodic, and vary from one trough to the next. This variability, in fact, is so important to genesis that NHC/TPC forecasters (for better or worse) in their online discussion regard each individual trough as a "tropical wave". We do not expect a uniform train of cyclonic cat's eyes but rather a zonally nonuniform pattern of cyclonic eddies - eddies which, in some sense, are "shed" from the AEJ as a result of the wave propagation and interaction with the mean flow at the critical latitude. Our first hypothesis concerning the role of the tropical wave critical layer in TC genesis is therefore

H1. Proto-vortex cyclonic eddies instrumental in TC formation are intimately associated with the parent wave's critical latitude in the lower troposphere. The critical layer and Kelvin cat's eye within, formed as a result of the wave's finite-amplitude interaction with its own critical latitude, contain a region of cyclonic rotation and weak straining/shearing deformation in which synoptic waves and mesoscale vorticity anomalies, moving westward together, amplify and aggregate on a nearly zero relative mean flow. This multi-scale interaction provides a dynamical pathway to "bottom-up" development of the proto-vortex from below.

The notion of bottom-up development (Halverson et al., 2007) is advanced as a counterpart to the more familiar "topdown" scenario advocated by Bister and Emanuel (1997) and Ritchie and Holland (1997), respectively; see also Ritchie et al. (2003). (This perspective was in vogue when M06 was written. The two scenarios are not mutually exclusive, as emphasized by Halverson et al.; see Glossary.) The top-down pathway begins with a recognition of the "top-heavy" nature of moist convective heating profiles in the presence of combined convective and stratiform precipitation processes. A top-heavy heating profile in a rotating environment necessar- ily leads to the conclusion that cyclonic vorticity is first concentrated in the middle troposphere by the induced vertical profile of convergence and must therefore find a way subsequently to burrow downward into the lower troposphere in order to provide the surface development needed for warmcore TC genesis. This pathway is inefficient (Tory and Montgomery, 2006) and invites a more direct route to genesis if one could be found, particularly in sectors where development is marginal to begin with. Observational findings of the top-down school were biased to the western Pacific warm pool, but in light of forecaster's experience we are inclined to regard this pathway as largely irrelevant to the Atlantic and eastern Pacific sectors outside the ITCZ ${ }^{12}$. The genesis of depressions in this region of the world is almost always associated with a surface low along the wave (see Reasor et al., 2005, for a case study); examples of mid-level cyclonic vortices in developing tropical systems, without a corresponding cyclonic signature near the surface, are evidently rare. Bottom-up development is inextricably linked to column moistening, which favors convective over stratiform heating as the leading contribution to the vertical heating profile. Column moistening, in turn, is naturally favored in a cyclonic critical layer. Our next hypothesis expands on this theme:

H2. The critical-layer cat's eye of the parent wave provides a set of quasi-closed material contours inside of which air is repeatedly moistened by convection, protected to some degree from lateral intrusion of dry air and impinging vertical shear, and (thanks to its location near the critical latitude) able to keep pace with the parent wave until the proto-vortex has strengthened into a self-maintaining entity.

This hypothesis is a "coin with two sides," combining the notion of repeated column moistening with the equally important notion that the Kelvin cat's eye is protected from lateral entrainment of air - air which, in the case of the AEJ, is often dry and dusty if associated with the Saharan Air Layer in the lower free troposphere (Thorncroft et al., 2003; Dunion and Velden, 2004; Wong and Dessler, 2005). Hypothesis 2 also adds the notion of rapid vertical transport by convection to what otherwise has been, in our discussion thus far, a description of moist barotropic or shallow-water disturbances superposed on an easterly off-equatorial jet. These quasi2-D disturbances organize the flow laterally via parcel displacement and create kinematic boundaries to lateral mixing; they also organize moist convection that lofts moisture into the free troposphere. Such convection, of course, is ubiquitous in the tropics and represents the primary mechanism of vertical transport that is rapid in comparison to other vertical motions such as isentropic up-glide along frontal zones

\footnotetext{
${ }^{12}$ Although the single developing system (Guillermo) observed during TEXMEX was originally thought to contain a low-level anticyclone in its earliest stage, subsequent analysis revealed a cyclonic circulation all the way to the surface (Raymond et al., 1998).
} 
and diabatic subsidence in the Hadley circulation caused by radiative cooling. In cases where the ambient cyclonic vorticity and moisture are positively correlated above the boundary layer, gyre formation is accompanied by horizontal entrainment of moisture, shortening the time required to approach column saturation. The "quasi" closure of streamlines or trajectories in $\mathrm{H} 2$ conveys two important caveats that will apply in all of the discussion to follow: (i) there is always a temporal development of the closure, as local wave amplitude grows and parcels become irreversibly entrained in the gyre, and (ii) there is often a nonzero divergent component of motion across the outer edge of a "closed" gyre as defined by the bounding contour of (nondivergent) stream function. We have greater confidence in the rotational component of motion than the divergent part, quantitatively speaking, and therefore attach greater significance to the appearance of a closed gyre in stream function. This is not to say that the analyzed divergent wind is unreliable; its sign usually agrees with the expected inflow (outflow) of mass in the lower (upper) troposphere in the neighborhood of a developing storm. At lower levels the divergent component of motion therefore contributes to the entrainment of vorticity and moisture as does the temporal development of the rotational component.

We do not limit deep convection to the critical layer (!) but advance the notion that convection within the Kelvin cat's eye is likely to create a vertical profile of diabatic heating with a lower- to mid-tropospheric maximum (Tory et al., 2006a, b, 2007) instead of the more widely observed "top-heavy" heating profile of canonical mesoscale convective systems (Houze, 1989; Mapes and Houze, 1995). The canonical profile represents a combination of convective and stratiform effects in which evaporative cooling in adjacent stratiform anvil regions offsets the low-level heating in deep convective cores. These two components superpose constructively in the mid- to upper troposphere. A top-heavy profile is important to the tropical general circulation (Hartmann et al., 1984), the mature phase of MJO (Lin et al., 2004) and generation of stratospheric gravity waves (D. Ortland, personal communication) but inimical to tropical cyclogenesis. The argument favoring a convective heating profile in TC genesis rests on an inference by Tory et al. (2007) that simulated storms initialized in actual environments (real-life examples) are accompanied by such a profile when development ensues, and vice versa. This profile also can be inferred indirectly from the observed divergent circulation response (Sect. 4.3) or "directly" from TRMM retrievals in the recirculating gyre (Dunkerton et al., 2009a). The heating profile is coupled to column moistening, and vice versa. As a region of re-circulating quasi-horizontal flow, the Kelvin cat's eye is bounded by closed material contours - a Lagrangian concept that provides a more general definition of the TC "pouch" in cases where a critical layer is not well defined or consists of a solitary shed vortex (as illustrated in Fig. 3; for further commentary on this figure see Appendix A5). The cat's eye is expected to trap, that is, to contain moisture or moist entropy lofted by convection. The net heating and its vertical profile depends, in turn, on the amount of moisture lofted. Lower tropospheric air close to saturation is less likely to experience evaporative cooling, and the cat's eye (for purely kinematic reasons) favors maintenance of nearly saturated conditions, especially if there exists a concentrated and sustained population of shallower "congestus" type convection in such a region, as suggested by some recent observations. For low-level mass convergence and vorticity amplification, a convective type of heating profile in the lower troposphere (LT) critical layer - without significant stratiform heating/cooling, or with less stratiform component than typical elsewhere - favors bottom-up development (M06) and concentration of upward mass flux in the lower troposphere (Raymond and Sessions, 2007). This is not to say that midlevel spin-up might not also occur in such cases, but to suggest that a low-level scenario is plausible in which the midlevel spin-up is unnecessary or of secondary importance to TC genesis ${ }^{13}$. This theoretical notion and its implications agree with the common observation by forecasters of a "surface low along the wave" in the development of a tropical depression.

Routine inspection of satellite water vapor imagery suggests that genesis is always associated with a relatively moist free troposphere as observed in the 6.7 micron band. There are many mechanisms governing the distribution of moisture; formation of a closed gyre in a tropical wave critical layer is but one of these. Quasi-stationary tropical features and extratropical disturbances also play a role. A developing gyre entrains whatever moisture exists nearby and experiences selfmoistening via deep convection within. Once moistened, it is desirable that the gyre (or some portion of it) remain so in order to facilitate TC formation. A key property of the protovortex required for subsequent development is its resilience to lateral intrusions of dry air which are common throughout the Atlantic during the hurricane season and at other times and places. The proto-vortex of a nascent TC, however, lacks a strong transport barrier (cf. McIntyre, 1989, 1993; Montgomery and Kallenbach, 1997; Bassom and Gilbert, 1999; Moulin and Flor, 2005) and is vulnerable to dry-air intrusion and vertical shear (e.g., Reasor et al., 2004). It therefore needs help from the parent wave. The protective kinematics of the Kelvin cat's eye (moving with the wave at its critical latitude) implies a reduction of dry air intrusions from outside (Tuleya and Kurihara, 1981) and a tendency for the heat released by cumulus convection to be retained in the comoving frame (Krishnamurti et al., 1994). The observation by Krishnamurti et al. is incorporated in a third hypothesis below.

\footnotetext{
${ }^{13}$ ERA-40 data examined here suggest, for what it is worth, that mid-level convergence resolved by the analyses occurs in developing systems but is secondary to low-level convergence chronologically as the proto-vortex develops upward.
} 
(a) $\mathrm{C}_{\mathrm{r}}=4 \mathrm{~m} / \mathrm{s}$

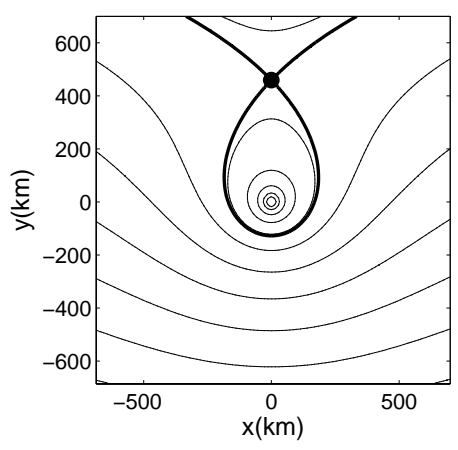

(b) $\mathrm{C}_{\mathrm{r}}=0 \mathrm{~m} / \mathrm{s}$

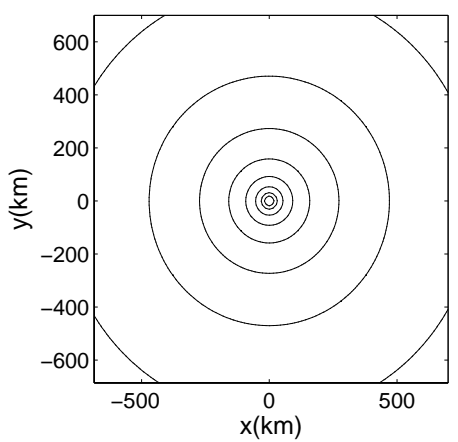

(c) $\mathrm{C}_{\mathrm{r}}=-4 \mathrm{~m} / \mathrm{s}$

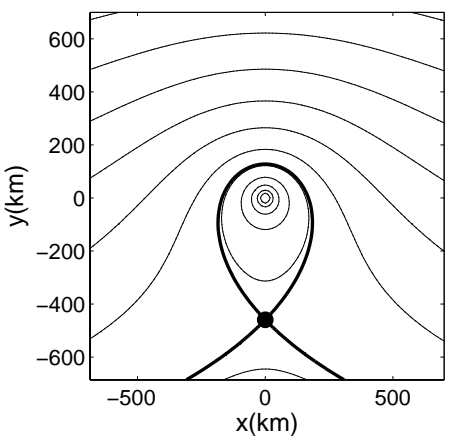

Fig. 3. Total streamline structure of a point vortex with cyclonic circulation $\Gamma$ immersed in a constant mean zonal flow $U_{b}$. In the frame of reference displayed here (with zonal translation speed equal to zero) the vortex is spatially and temporally fixed at the origin. The total flow is given by the superposition of a constant background zonal wind and a circularly symmetric flow induced by the point vortex. This flow is an exact steady solution of the 2-D Euler equations. As noted in the text, identical streamline patterns are obtained in a resting basic state when the vortex translates zonally at speed $C_{p}=-U_{b}$. The numerical value of $\Gamma$ is based on the gyre circulation analyzed for pre-Debby in August 2000. Three cases are sketched: (a) $U_{b}=4 \mathrm{~ms}^{-1}$ (moderate westerly flow over the vortex), (b) $U_{b}=0$ (zero background flow) and (c) $U_{b}=-4 \mathrm{~ms}^{-1}$ (moderate easterly flow). Light solid curves denote streamlines. The black dot denotes the stagnation point located at $\left(X_{c}, Y_{c}\right)=\left(0, \Gamma / 2 \pi U_{b}\right)$; the fluid velocity vanishes there. The thick solid curve denotes the dividing streamline that divides the flow into three distinct regions: a core region near the vortex, upper outside region and lower outside region. As the magnitude of the imposed flow increases, the distance from the vortex center to the separatrix decreases and the amount of fluid isolated within the core region decreases.

According to the marsupial paradigm, the critical layer guarantees some measure of protection from intrusion. But actual flow fields are transient and contain mesoscale finestructure, making the Lagrangian kinematics rather messy. A group of smaller vortices, e.g., will entrain the surrounding air more readily than a single larger vortex ${ }^{14}$. As for how these smaller vortices are created in the first place, there are essentially two possibilities: (i) upscale aggregation of mesoscale convective vortices associated with MCSs and/or VHTs, and (ii) eddy shedding that works its way to smaller scales via a forward enstrophy cascade, such as might be associated with wave breaking at the critical layer. Tropical cyclogenesis evidently represents a kind of process in which the inverse energy and forward enstrophy cascades (originating respectively from cloud system and synoptic scales) collide in "spectral" space at some intermediate scale to form a diabatic vortex larger in horizontal scale than the vortices associated with individual cloud systems but substantially smaller in scale than the mother pouch created by the synoptic wave. This GFD aspect is perhaps the most fascinating and daunting of TC genesis; one that has not yet been fully explored (owing to limitations of horizontal resolution in observations or models) but may be advanced as a framework for understanding the multi-scale nature of the problem. Of relevance to $\mathrm{H} 2$ are the following considera-

\footnotetext{
${ }^{14}$ Relevant to this discussion are two studies outside the atmospheric literature. The effects of entrainment on the buoyancy of rotating Bénard convection were discussed by Julien et al. (1999). Organization of deep ocean convection by mesoscale eddies was examined by Legg et al. (1998).
}

tions: (i) A group of cloud-system vortices at meso- $\gamma$ may delay saturation of the column by a single master VHT, by mixing drier area within the pouch with moister air lofted by the VHT. (ii) A group of mesoscale vortices (resulting either from dry or moist processes at meso- $\beta$ ) may entrain drier air from outside the pouch via chaotic advection, partially offsetting the cumulative moistening of this area by persistent deep convection. Requirements for horizontal resolution differ depending on which of these mechanisms is more relevant; they are less stringent for chaotic advection than for upscale aggregation.

A third hypothesis is motivated by the fact that the Kelvin cat's eye of a critical layer is where local sources or sinks of momentum and entropy are amplified owing to the fact that parcels spend a considerably longer time in such regions compared to those well outside the critical layer. In linear theory the net effect of fixed sources and sinks is inversely proportional to intrinsic frequency. This response, as noted by Held and Ting (1990), contrasts sharply with topographic wave excitation in which the response is proportional to the mean flow speed relative to the obstacle. It exists for purely kinematic reasons and is entirely independent of any presumed relationship between the wave and induced sources or sinks. In fact, since the wave trough is a preferred region for diabatic heating in tropical waves (or close to such; see Kiladis et al., 2006) and its longitude is at the center of the cat's eye (Fig. 1) the effect of diabatic heating due to deep moist convection is doubly amplified in this region of the critical layer. (i) The heating is stronger here (owing primarily to mesoscale processes that favor convection in the trough) and (ii) its rectified effect on the wave is also stronger owing to 
the kinematics of parcel motion in the critical layer. Hence we propose that

H3. The parent wave is maintained and possibly enhanced by diabatically amplified eddies within the wave (proto-vortices on the mesoscale), a process favored in regions of small intrinsic phase speed.

This hypothesis, incidentally, agrees with the common observation that a tropical wave is weakened or eliminated when the diabatic vortex leaves the pouch, having acquired its own identify and propagation characteristics independent of the parent wave. A typical scenario ${ }^{15}$ is that a westward propagating wave carries its proto-vortex for a time, and if a depression ensues, the strengthened and enlarged vortex acquires a poleward component of propagation while its zonal component begins to slow relative to the phase speed of the parent wave. The parent wave continues its westward motion at more or less the same speed it originally had, but with severely diminished amplitude. In the Atlantic sector the wave speed is typically $4-6^{\circ}$ per day westward, while the vortex speed is $\sim 2^{\circ}$ per day west-northwestward. Individual cases may resemble this scenario or differ considerably; nevertheless, the basic description is robust.

The observed combination of westward moving tropical wave and proto-vortex within evokes a "marsupial" paradigm for TC genesis, as charmingly illustrated in the popular online encyclopedia ${ }^{16}$. In contemporary jargon the marsupial paradigm combines elements of a diabatic Rossby wave (Wernli and Kenzelmann, 2006) with those of a diabatic Rossby vortex (Moore and Montgomery, 2004, 2005, 2006). We suggest that the diabatically activated easterly wave is a hybrid wave/vortex structure, particularly in cases leading to formation of a tropical depression ${ }^{17}$.

\subsection{The role of vertical shear}

A mysterious and sometimes counter-intuitive element of the marsupial paradigm is the role of mean vertical shear in TC

\footnotetext{
${ }^{15}$ Sometimes two or more visible proto-vortices are involved, a scenario that may be more common in the western Pacific monsoon trough environment (Edson and Lander, 2006).

${ }^{16}$ http://en.wikipedia.org/wiki/Marsupial

${ }^{17}$ It is noteworthy that most tropical depressions become named storms, although the reasons are unclear. The cumulative experience of forecasters is evidently important in assigning depression status and in knowing at what formative level further development is virtually assured. This property of tropical depressions suggests a finite-amplitude instability for cyclogenesis above a certain threshold amplitude (e.g., Emanuel, 1989). As to what the relevant criteria are, they likely include some measure of tangential circulation strength, persistence of deep convection near the center, and a (possibly subjective) sense of closed circulation. Such criteria, in any case, must be invariant under a Galilean transformation, that is, Lagrangian properties of the flow. A coordinate-dependent Eulerian measure (e.g., westerlies south of center, in the resting frame) is inadequate.
}

genesis. It is widely appreciated, especially by forecasters, that strong vertical shear is inimical to genesis. This fact is thought to account for the reduction of Atlantic hurricanes in warm ENSO events, as the eastern Pacific "warm pool" forces upper-level westerlies over the Caribbean (Gray, 1984a, b; Goldenberg and Shapiro, 1996). Although reasons suggest themselves, none are entirely convincing. Vertical shear in the upper troposphere (UT) is sometimes imagined to "ventilate" deep convection, but since the water content is very small at these altitudes, thermodynamic consequences arising from the phase change of water in the UT are likely to be very small as well. Similarly, the clear-sky radiative impacts of water vapor redistribution in the UT are probably too slow to affect genesis on time scales of 1-2 days, although their effects on longer time scales (e.g., MJO) may be significant. An indirect effect of vertical shear, if westerly, is an implied wave duct for quasi-stationary waves of midlatitude origin in the tropical upper troposphere. This dynamical opening between tropics and extratropics (Webster and Holton, 1982) is responsible for the appearance of synopticscale troughs sometimes observed to penetrate the tropics in the hurricane season. If such "troughy" flow persists over a substantial part of the season it may ostensibly suppress genesis in some years (e.g., Atlantic 2006). The extratropical disturbances themselves may enhance genesis (TT pathway: Davis and Bosart, 2001; Hendricks et al., 2004; Hidalgo, 2007) or suppress it (via dry air intrusion or cyclonic flow aloft) so their net effect is not obvious and may be sensitive to the juxtaposition between upper- and lower-level disturbances (Davis and Bosart, 2004; Bosart, 2006).

It is well-known that vertical shear can be detrimental to hurricane intensification; the strongest and most circularly symmetric storms have little environmental shear or shear tendency. It is precarious, however, to take whatever mechanisms are responsible for the effects of shear on hurricanestrength vortices and assign them to the pre-depression stage. Whereas in a mature system, vertical shear interacts with a single large vortex, in genesis, the shear is interacting with one or more deep convecting elements, rotating or not (Frank and Johnson, 1969; Gentry et al., 1970; Hendricks et al., 2004; M06; Hendricks and Montgomery 2006). At this stage, the effects of shear are more likely to be felt in convective organization rather than via the dynamics of a single large vortex. We speculate that strong shear in the genesis stage (i) may lead to linear rather than circular organization, and that (ii) longitudinally radiating gravity waves or density currents coupled to convection are more likely to be excited when the LT shear is strong (LeMone et al., 1998). Mechanisms of convective organization in vertical shear were studied by Corbosiero and Molinari (2002) and Molinari et al. (2004, 2006). Forecasters' experience notwithstanding, there is no reason to think that genesis is optimized in exactly zero vertical shear. If it were, the fact that easterly-wave amplitudes maximize near the altitude of AEJ maximum $(\sim 600 \mathrm{hPa})$ would be convenient to the marsupial 
paradigm because wave + mean vertical shear is zero at this altitude, by definition. We suspect that the role of vertical shear in the marsupial paradigm is more subtle and may (in some cases) facilitate refraction of the waves, without affecting adversely their embedded deep convection.

\section{Morphology of waves and TC genesis in representa- tive cases}

Sixty-one named storms formed over the Atlantic (including the Caribbean Sea and Gulf of Mexico) and eastern Pacific during August and September 1998-2001, almost one-half of the 136 (named or numbered) tropical depressions that formed during all four hurricane seasons within these two ocean basins and their adjacent seas. Fifty-four (88\%) of the 61 named storms fell into the "monochromic easterly wave" category based on the criteria described in Appendix A3. In the remaining (seven) cases, either a multiple-wave interaction was involved, or easterly waves were not well-defined ${ }^{18}$. Among the 54 storms in the monochromatic category, many formed from African easterly waves; others over the eastern Pacific formed from wave disturbances originating in the ITCZ. Although unstable ITCZ disturbances have a different morphology from African easterly waves, as explained in Appendix C, they support the marsupial paradigm; genesis is found once again to occur in the Kelvin cat's eye of the wave's critical layer. In addition to the 54 cases from the Atlantic and eastern Pacific, we include a single case from the central Pacific just east of the Dateline $\left(178^{\circ} \mathrm{E}\right)$, bringing the total number of named storms in the monochromatic easterly wave category to 55. For each event a zonal phase speed was determined as a function of height in the lower troposphere, as described in Appendix A4. Key to the following analysis is our visualization of the horizontal flow (streamlines and stream function) in a frame of reference translating westward with the parent wave, at its speed of zonal phase propagation, as explained in Appendix A5.

\subsection{Easterly wave/TC genesis events}

The time of TC genesis, lat/lon location and accompanying wave phase speeds at 850 and $600 \mathrm{hPa}$ for the 55 "monochromatic easterly wave" cases are summarized in Table 1, together with the uncertainty of the phase speed estimate, and various properties of the flow in which the proto-storm develops, such as (i) the area of the surrounding closed gyre in the co-moving frame, (ii) orientation of the separatrix or dividing streamline that bounds the gyre, as defined by the direc-

\footnotetext{
${ }^{18}$ Our selection of quasi-monochromatic easterly wave cases highlights the role of incident waves in the lower, not upper, troposphere. Various UT phenomena such as midlatitude baroclinic waves, westward subtropical Rossby waves, and erosion of vertical shear by deep moist convection can play an important role in genesis, but are disregarded in the selection criteria which emphasize tropical waves in the LT.
}

tion of its saddle point ("hyperbolic point") relative to gyre center, and (iii) two measures of recirculation time about the proto-storm center, to be discussed in Sect. 4.1. For most of these quantities the median of the distribution is shown at the bottom, together with one-half the inter-quartile range, representing the difference between the 75th and 25 th percentiles, divided by two.

Phase speeds are similar at the two levels, though slightly larger in median at $600 \mathrm{hPa}$. About $60 \%$ of the members have larger phase speed at $600 \mathrm{hPa}$, a bias that seems somewhat more prevalent in the eastern Pacific. A physical interpretation is that some of the waves in question are not modes with fixed vertical structure, but a sheared disturbance or quasimode with vertical alignment that varies with time. Evident in the resting frame is that waves faster than $\sim 7 \mathrm{~ms}^{-1}$ westward are found only in the Atlantic sector, although slower waves are found here, too, similar to those of the eastern Pacific. The ratio of 600 to $850 \mathrm{hPa}$ phase speed is generally larger for slower waves than for faster waves.

The uncertainty of phase speed was obtained performing a least-squares fit on the Hovmöller diagram in up to four ways: fitting the positive and negative lobes of band-pass meridional velocity anomaly individually, either (i) 3 days prior to genesis, or (ii) (2 days prior) + (1 day after) genesis. The latter contingency allows a reliable estimate in a few cases where a well-defined wave did not exist more that $48 \mathrm{~h}$ before genesis. We are naturally reluctant to use wave properties after genesis to estimate gyre kinematics before genesis because the wave morphology and propagation begin to change when a tropical depression is born, and significantly so, once the "joey" leaves the "pouch". For this reason the phase speed estimates and uncertainty thereof were based primarily on the pre-genesis behavior of the waves.

Gyre properties such as size and orientation vary widely among the 55 cases selected for study, whereas the estimates of recirculation time occupy a much narrower range, probably because (as explained in Sect. 4.1) they are derived from dynamical information closer to the center of the gyre. It should be kept in mind that gyre size, as displayed here, is a metric of area: e.g., the value 25 is approximately equivalent to a $5 \times 5$ array of grid points, the side of each grid box equal to $\sim 1.125^{\circ}$. A linear metric would reveal a narrower range, to be sure, but the range of gyre areas is impressive nonetheless (varying by a factor of 200). Given that the Atlantic and eastern Pacific storms are usually modest in extent (unlike western Pacific typhoons of the tropical depression category) relative to the larger gyres observed in these two sectors, a reasonable preliminary conclusion is that, beyond a certain size, a very large gyre area is not relevant to storm development. Regarding separatrix orientation, a pronounced dearth of angles occurs near $-90^{\circ}$ (due south) - a key observation for identifying the Lagrangian flow around the proto-storm and the air mass from which environmental properties have been entrained. We emphasize this point in the examples to follow. 
Table 1. Statistics of the 55 storms during August-September 1998-2001. The left four columns show the storm names (highlighted cases in red), genesis time (UTC-day-month-year), genesis latitude (Lat) and longitude (Lon). Other columns list the phase speed $\left(C_{p}\right)$ of the easterly waves, its standard deviation $\left(\delta C_{p}\right)$ based on 2-4 estimates, the gyre size (in terms of grid points in T106 ECMWF data), the angle of the separatrix, and recirculation time $\tau_{1}$ and $\tau_{2}$ (units: days) at $850 \mathrm{hPa}$ and $600 \mathrm{hPa}$. (-) indicates an undefined value. The phase speed and its standard deviation are derived from the Hovmöller diagrams (see Appendix A3 for details). The orientation of the separatrix is defined as the angle between the line from the gyre center to the nearest saddle point on the gyre's bounding streamline and the east direction ( 90 indicates that the saddle point is due north of the gyre center, 180 indicates due west of the gyre center, and so on). The recirculation time $\tau_{\zeta}$ is defined as

$\tau_{\zeta}=4 \pi / \zeta_{\max }$

where $\zeta_{\max }$ is the maximum vorticity within the gyre. The recirculation time $\tau_{o w}$ is defined as

$\tau_{o w}=4 \pi / \sqrt{\overline{O W}}$

where $\overline{O W}$ is the average of positive Okubo-Weiss in the regions with cyclonic vorticity within the gyre. If a gyre does not exist, $\zeta_{\text {max }}$ and $\overline{O W}$ are defined as vorticity and Okubo-Weiss at the genesis location, respectively. For negative $\zeta_{\max }$ or $\overline{O W}$, the recirculation is undefined. The gyre is based on the 36-h pre-genesis average of stream function in the co-moving frame of reference. (see Sect. 4.1 for details). Values at genesis time are substituted where the 36-h gyre does not exist (magenta). The median and one-half inter-quartile range of phase speed, gyre size and recirculation time are shown in the bottom two rows.

\begin{tabular}{|c|c|c|c|c|c|c|c|c|c|c|c|c|c|c|c|}
\hline \multirow[b]{2}{*}{ name } & \multirow[b]{2}{*}{ genesis time } & \multirow[b]{2}{*}{ Lat } & \multirow[b]{2}{*}{ Lon } & \multicolumn{6}{|c|}{$850 \mathrm{hPa}$} & \multicolumn{6}{|c|}{$600 \mathrm{hPa}$} \\
\hline & & & & $\mathrm{Cp}(\mathrm{m} / \mathrm{s})$ & $\mathrm{dCp}(\mathrm{m} / \mathrm{s})$ & gyre size & s'trix angle & $t_{z}$ (day) & $t_{\text {ow }}$ (day) & $\mathrm{Cp}(\mathrm{m} / \mathrm{s})$ & $\mathrm{dCp}(\mathrm{m} / \mathrm{s})$ & gyre size & s'trix angle & $t_{z}$ (day) & $t_{o w}$ (day) \\
\hline Jeanne & 06Z 21 Sep 1998 & 9.6 & -17.4 & -4.1 & 1.7 & 33 & 8 & 1.4 & 2.3 & -3.5 & - & 63 & -2 & 2.1 & 4.0 \\
\hline Alberto & $18 \mathrm{Z} 03$ Aug 2000 & 10.8 & -18.0 & -8.4 & 1.1 & 23 & 6 & 6.4 & 9.4 & -11.1 & 1.0 & 23 & 18 & 4.7 & 8.5 \\
\hline Cindy & 00Z 19 Aug 1999 & 13.5 & -18.9 & -7.8 & 1.4 & 14 & -70 & 5.4 & 8.9 & -6.5 & 0.6 & 185 & 165 & 2.0 & 7.3 \\
\hline Isaac & $12 \mathrm{Z} 21 \mathrm{Sep} 2000$ & 11.5 & -23.0 & -8.7 & 0.9 & 9 & 54 & 2.4 & 4.4 & -6.3 & 0.4 & 109 & 7 & 2.9 & 5.3 \\
\hline Gert & 12Z 11 Sep 1999 & 12.6 & -24.2 & -8.5 & 0.6 & 87 & 56 & 2.4 & 4.4 & -8.6 & 0.5 & 78 & 170 & 3.0 & 5.4 \\
\hline Georges & 12Z 15 Sep 1998 & 9.7 & -25.1 & -6.2 & 0.2 & 25 & 55 & 4.3 & 6.8 & -6.3 & 1.4 & 39 & -55 & 5.2 & 8.9 \\
\hline Ivan & OOZ 19 Sep 1998 & 13.4 & -26.6 & -4.4 & 2.1 & 21 & 57 & 2.1 & 3.4 & -4.6 & 0.7 & 38 & -3 & 3.0 & 6.3 \\
\hline Felix & $18 \mathrm{Z} 07 \mathrm{Sep} 2001$ & 13.9 & -28.4 & -5.5 & 0.8 & 22 & 170 & 1.5 & 3.0 & -6.6 & 1.3 & 75 & 158 & 2.9 & 5.4 \\
\hline Joyce & $12 Z 25$ Sep 2000 & 11.2 & -29.6 & -6.6 & 0.9 & - & - & 10.1 & - & -6.6 & 0.7 & - & - & 13.1 & 13.7 \\
\hline Danielle & 06Z 24 Aug 1998 & 13.4 & -34.3 & -8.3 & 1.0 & 9 & 56 & 5.2 & 7.6 & -8.5 & 0.4 & 115 & 98 & 3.8 & 8.2 \\
\hline Erin & $18 \mathrm{Z} 01 \mathrm{Sep} 2001$ & 12.5 & -34.3 & -10.7 & 0.9 & - & - & 5.4 & 6.4 & -11.1 & 0.5 & 15 & 134 & 4.4 & 6.9 \\
\hline Chantal & 18Z 14 Aug 2001 & 12.8 & -37.0 & -7.5 & 4.1 & 1 & -5 & 2.6 & 3.0 & -10.2 & 0.65 & -7 & 4.5 & 8.2 & \\
\hline Debby & $18 \mathrm{Z} 19$ Aug 2000 & 12.0 & -44.5 & -10.0 & 0.6 & 33 & 36 & 3.8 & 6.6 & -9.1 & 0.3 & 120 & 56 & 4.0 & 7.4 \\
\hline Ernesto & $12 \mathrm{Z} 01 \mathrm{~S}$ ep 2000 & 14.8 & -45.2 & -8.3 & 0.14 & 29 & 5.4 & 8.0 & -6.1 & - & 35 & 14 & 7.2 & 11.9 & \\
\hline Floyd & 18Z 07 Sep 1999 & 14.6 & -45.6 & -7.3 & 0.4 & 65 & 70 & 3.3 & 5.3 & -6.9 & 0.1 & 60 & -4 & 3.1 & 5.7 \\
\hline Bonnie & 12Z 19 Aug 1998 & 14.7 & -48.1 & -8.0 & 0.7 & 114 & 9 & 2.9 & 5.2 & -8.3 & 0.2 & 79 & 124 & 3.6 & 6.3 \\
\hline Chris & $12 Z 17$ Aug 2000 & 14.2 & -51.9 & -9.1 & 1.1 & - & - & 5.9 & 7.8 & -8.5 & 0.5 & 3 & 73 & 4.8 & 7.5 \\
\hline Helene & $12 \mathrm{Z} 15 \mathrm{Sep} 2000$ & 14.9 & -52.2 & -10.2 & 0.2 & - & _- & 9.0 & 12.1 & -8.7 & 0.1 & 1 & -63 & 12.2 & 15.6 \\
\hline Emily & 06Z 24 Aug 1999 & 11.5 & -53.6 & -4.3 & 0.1 & 9 & 37 & 3.2 & 6.7 & -4.1 & 0.5 & 9 & 33 & 3.4 & 6.8 \\
\hline Humberto & $12 Z 21$ Sep 2001 & 25.1 & -64.2 & -4.5 & 1.0 & 12 & 125 & 4.2 & 6.6 & -3.3 & 0.2 & 3 & 81 & 5.3 & 7.2 \\
\hline Dean & $12 Z 22$ Aug 2001 & 17.9 & -64.3 & -5.0 & 0.2 & - & - & 25.6 & - & -8.4 & 1.6 & - & - & - & - \\
\hline Dennis & 00Z 24 Aug 1999 & 21.5 & -67.7 & -3.7 & 0.9 & 9 & -119 & 3.2 & 4.9 & -4.5 & 1.4 & 15 & -132 & 3.1 & 4.7 \\
\hline Florence & $18 \mathrm{Z} 10$ Sep 2000 & 30.9 & -70.9 & -1.9 & 0.6 & 43 & -134 & 2.7 & 4.8 & -3.0 & 0.0 & 6 & 45 & 2.4 & 4.0 \\
\hline Barry & $12 Z 02$ Aug 2001 & 25.7 & -84.8 & -2.4 & 0.2 & 5 & -96 & 3.1 & 3.1 & -7.7 & 4.4 & 19 & 94 & 4.0 & 3.8 \\
\hline Gordon & $12 \mathrm{Z} 14 \mathrm{Sep} 2000$ & 19.8 & -87.3 & -2.5 & 1.2 & 7 & -171 & 4.8 & 7.8 & -3.8 & 0.0 & 16 & -174 & 7.0 & 7.3 \\
\hline Hermine & 12Z 17 Sep 1998 & 26.9 & -90.3 & -2.8 & 0.6 & 14 & 180 & 3.0 & 4.2 & -3.9 & 0.1 & 71 & 147 & 2.9 & 5.4 \\
\hline Juliette & 06Z 21 Sep 2001 & 12.6 & -91.1 & -7.0 & 1.0 & 76 & 98 & 4.5 & 10.6 & -7.9 & 0.8 & 78 & -173 & 3.3 & 6.7 \\
\hline Charley & 06Z 21 Aug 1998 & 25.3 & -92.3 & -4.1 & 0.5 & 66 & -39 & 4.1 & 6.3 & -6.5 & 1.0 & 103 & 68 & 4.5 & 7.7 \\
\hline Beryl & $18 \mathrm{Z} 13$ Aug 2000 & 22.5 & -93.5 & -3.8 & 2.8 & 10 & 50 & 3.7 & 4.6 & -3.1 & 0.8 & 25 & 62 & 4.2 & 5.1 \\
\hline Earl & 12Z 31 Aug 1998 & 21.6 & -93.5 & -2.5 & 0.9 & 68 & -177 & 1.9 & 3.1 & -5.6 & 1.0 & 42 & 155 & 2.7 & 4.3 \\
\hline Bret & 18Z 18Aug 1999 & 19.5 & -94.4 & - & - & 1 & 58 & 14.0 & - & -3.3 & 1.0 & 17 & -137 & 4.3 & 7.4 \\
\hline Frances & 18Z 08 Sep 1998 & 25.5 & -94.5 & -5.5 & - & 47 & 151 & 2.9 & 4.6 & -3.2 & 1.0 & 211 & 119 & 2.2 & 5.3 \\
\hline Ivo & $12 Z 10$ Sep 2001 & 14.8 & -98.9 & -6.6 & 0.5 & - & - & 8.6 & - & -6.9 & 2.2 & 7 & 162 & 3.5 & 7.8 \\
\hline Dora & 00Z 06 Aug 1999 & 12.1 & -100.9 & -2.9 & 0.3 & 22 & 5 & 1.9 & 3.0 & -4.5 & 1.0 & 63 & 160 & 3.7 & 8.1 \\
\hline Lane & 00Z 05 Sep 2000 & 15.4 & -102.2 & -4.0 & 1.1 & 24 & 138 & 1.2 & 1.8 & -3.3 & 0.5 & 24 & 155 & 1.6 & 2.6 \\
\hline Ileana & $18 \mathrm{Z} 13$ Aug 2000 & 17.1 & -104.0 & -4.9 & 0.5 & 11 & 105 & 3.2 & 4.7 & -4.7 & 0.1 & 25 & -174 & 1.9 & 3.6 \\
\hline Greg & 12Z 05 Sep 1999 & 18.6 & -105.1 & -2.8 & 0.2 & 35 & 90 & 1.5 & 2.7 & -3.3 & 0.6 & 142 & 153 & 1.9 & 4.4 \\
\hline Gilma & 00Z 05 Aug 2000 & 15.0 & -105.2 & -3.7 & 0.8 & 18 & 157 & 1.9 & 3.1 & -4.1 & 0.7 & 38 & 153 & 1.9 & 3.3 \\
\hline Hector & $18 \mathrm{Z} 10$ Aug 2000 & 17.8 & -106.6 & -5.1 & 0.3 & 15 & 105 & 6.5 & 6.8 & -6.1 & 0.9 & 216 & 156 & 3.4 & 7.7 \\
\hline Javier & 12Z 06 Sep 1998 & 17.8 & -106.8 & -3.0 & 0.3 & 47 & 102 & 2.5 & 4.5 & -5.0 & 0.4 & 37 & 172 & 2.2 & 4.6 \\
\hline Hilary & 06Z 17 Sep 1999 & 15.2 & -107.1 & -2.6 & 0.4 & 23 & 171 & 1.0 & 1.8 & -2.8 & 0.3 & 54 & -11 & 1.8 & 3.3 \\
\hline Miriam & $18 \mathrm{Z} 15 \mathrm{Sep} 2000$ & 19.2 & -107.4 & -3.2 & 0.0 & 3685 & 5.0 & 8.0 & -3.9 & 0.7 & 99 & 31 & 3.8 & 6.7 & \\
\hline Flossie & 06Z 26 Aug 2001 & 19.1 & -108.5 & - & - & 1 & 157 & - & 14.8 & -5.3 & 0.6 & 223 & 66 & 3.0 & 5.4 \\
\hline Henriette & $12 Z 04$ Sep 2001 & 16.9 & -108.8 & -2.8 & 0.5 & 88 & 136 & 1.1 & 2.1 & -4.3 & 1.3 & 20 & -176 & 1.9 & 3.0 \\
\hline Georgette & O0Z 11 Aug 1998 & 11.0 & -108.9 & -5.8 & 1.4 & 36 & 125 & 1.9 & 3.6 & -7.1 & 0.2 & 6 & 23 & 3.9 & 6.2 \\
\hline Isis & 00Z 01Sep 1998 & 18.3 & -109.2 & -3.2 & 1.1 & 79 & 91 & 1.6 & 2.5 & -3.9 & 1.6 & 88 & -172 & 1.8 & 3.4 \\
\hline Frank & 12Z 06 Aug 1998 & 16.7 & -111.5 & -4.2 & 0.3 & 89 & 82 & 3.3 & 7.3 & -5.8 & 1.6 & 120 & 164 & 3.1 & 5.8 \\
\hline Fernanda & 06Z 17 Aug 1999 & 12.4 & -113.1 & -5.1 & 0.7 & 47 & 154 & 3.1 & 4.5 & -5.3 & 0.2 & 76 & -103 & 2.9 & 5.1 \\
\hline Fabio & $12 Z 03$ Aug 2000 & 16.4 & -113.6 & -3.7 & 0.5 & 78 & 65 & 1.5 & 2.8 & -3.8 & 0.6 & 64 & -8 & 1.4 & 2.9 \\
\hline Kiko & $18 \mathrm{Z} 21$ Sep 2001 & 15.6 & -116.1 & -5.8 & 0.7 & 80 & 139 & 1.5 & 3.1 & -5.3 & 0.6 & 85 & -20 & 2.1 & 4.0 \\
\hline Eugene & 06Z 06 Aug 1999 & 12.2 & -119.9 & -3.5 & 0.4 & 18 & 97 & 3.0 & 5.4 & -5.8 & 1.2 & 63 & 92 & 3.3 & 6.6 \\
\hline Gil & 06Z 04 Sep 2001 & 15.4 & -122.6 & -3.1 & 0.9 & 41 & 176 & 2.6 & 5.6 & -2.4 & 0.8 & 5 & 4 & 3.2 & 3.7 \\
\hline Kristy & 00Z 31 Aug 2000 & 13.0 & -131.4 & -3.2 & 1.0 & 25 & -9 & 2.1 & 3.8 & -2.1 & 0.9 & 11 & 6 & 2.6 & 4.4 \\
\hline John & 06Z 28 Aug 2000 & 14.9 & -137.4 & -3.1 & 0.3 & 27 & -167 & 1.7 & 3.8 & -2.7 & 0.3 & 26 & 178 & 2.4 & 4.3 \\
\hline Shanshan & $18 \mathrm{Z} 15 \mathrm{Sep} 2000$ & 15.1 & -182.0 & -2.8 & 0.3 & 70 & -171 & 2.6 & 4.9 & -5.7 & 2.8 & 16 & 152 & 3.0 & 4.9 \\
\hline median & & -4.4 & & 25 & & 3.1 & 4.7 & -5.3 & & 39 & & 3.1 & 5.6 & & \\
\hline $\mathrm{IQR} / 2$ & & 1.9 & & 18 & & 1.4 & 1.8 & 1.5 & & 32 & & 0.8 & 1.5 & & \\
\hline
\end{tabular}


Noting a wide range of sizes at both levels, the $600 \mathrm{hPa}$ gyres are systematically larger, but with a proportionately larger range. There are several examples of late-developing gyres (magenta). Allowing for inclusion of these cases to fill gaps left by the 36-h statistics, all but two of the storms have closed translating gyres at $600 \mathrm{hPa}$. This number is reduced to one (Dean) for reasons explained in Sect. 3.3 and footnote 24. There are several storms with no apparent gyre at $850 \mathrm{hPa}$ - still a small fraction of the total - which seems consistent, at least, with the tendency for gyres to be smaller at this level and therefore more vulnerable to fall below the finite horizontal resolution of the analyses. A few gyres (mostly at $850 \mathrm{hPa}$ ) contain only one grid point; for these cases a $3 \times 3$ grid box was substituted for the purpose of calculating gyre averages as described in Sect. 4.3.

The time and longitude of events provides an important clue regarding the possible role of hydrodynamic instability in tropical cyclogenesis which may be expected to give birth to paired systems within a short time interval (Ferreira and Schubert, 1997). There are 4 paired events in Table 1 ( 1 in the mid-Atlantic, 3 in the eastern Pacific) where both depressions were declared within $24 \mathrm{~h}$, and 15 paired events where both were declared within 6 days - all such events occurring within $20^{\circ}$ longitude of each other. Amazingly these events (whether combined or segregated by basin) can be modeled by a linear regression of longitude vs time separation with slope (phase speed) of -4.2 to $-4.6 \mathrm{~ms}^{-1}$ and $y$-intercept (zonal wavelength) of 17.1 to $17.6^{\circ}$ longitude, with extremely high $R^{2}$ values ( 0.88 to 0.91 ). But whereas this model seems to explain the paired systems of the eastern Pacific quite well, most of the Atlantic waves in the paired group have phase speeds about twice as fast as the model estimate. Pairing does not suffice to prove instability; it simply indicates that a wave packet had two adjacent gyre-troughs favorable to genesis. In theoretical terms the question is whether the ITCZ supports "absolute" instability that may occur in situ without an upstream stimulus (Pierrehumbert, 1984). We cannot answer this question here. Nevertheless, the observed pairing of genesis events strengthens the marsupial paradigm by showing that large-scale organization of rotating convection spans more than a single wavelength of incident waves.

All 55 wave/genesis events have been examined, and our conclusion is that in most cases (i) persistent convective precipitation occurs in a quasi-closed recirculating gyre in the co-moving frame, and (ii) the tropical storm develops near the intersection of the critical latitude and the trough axis. To illustrate our basic results, five representative storms during August 2000 were selected; the evolution of easterly waves and convection (as measured by TRMM accumulated precipitation) before TC genesis are discussed in detail for these cases.

\subsection{Illustrative cases from August-September 2000}

Hovmöller diagrams of 2.5-9 day bandpass meridional velocity at the best-track genesis latitude for each of the five highlighted cases are shown in Fig. 4 at the 850 and $600 \mathrm{hPa}$ levels. The large dot near the center of each panel indicates the longitude and time of genesis according to the best-track data, while filled squares trace the storm's longitude subsequent to genesis, at 6-h intervals. (The latitude of the storm is not shown here; it is listed in Table 1 and shown in the maps to follow.) Each storm originated between a negative and positive meridional velocity anomaly to the west and east, respectively, a region of anomalous cyclonic vorticity. In the first four examples, easterly waves at the two levels were aligned almost perfectly in the vertical, while in the remaining case (Shanshan) an eastward phase tilt with height can be detected. The tilt diminishes approaching genesis time as the $600 \mathrm{hPa}$ wave (with approximately twice the phase speed: Table 1) catches up with the $850 \mathrm{hPa}$ wave. In such cases an improving vertical alignment of the wave is part of the pre-genesis sequence. Characteristic of these examples and almost all of the events in Table 1 is that easterly wave anomalies (i) are present prior to genesis time, (ii) are reasonably monochromatic for at least the two preceding days, and (iii) become well aligned vertically in the lower troposphere below $500 \mathrm{hPa}$ before genesis occurs. The fact that wave anomalies appear vertically aligned does not imply, however, that the flow kinematics are identical at 850 and $650 \mathrm{hPa}$, as will be evident in some of the cases highlighted.

Of the ten panels shown in Fig. 4, only the first two display a reasonably uniform pattern of wave amplitude and zonal phase propagation across the entire longitude range and time period spanned by the figure. It turns out that pictures like Fig. $4 \mathrm{a}, \mathrm{b}$ are the exception and not the rule. Most often we find one or more of the following in the Hovmöller plots of meridional velocity anomaly: (i) a group of wave phases originating to the west (east) of the genesis location, followed by eastward (westward) apparent group propagation across the diagram (the eastward scenario is preferred and nicely illustrated in Fabio and Shanshan); (ii) abrupt amplification of the wave packet near the genesis point (as in Beryl); (iii) diminution of wave amplitude towards the end of the storm track (in all panels except Shanshan $850 \mathrm{hPa}$ ) and (iv) a pronounced wave packet in the upper right quadrant of the Hovmöller diagram (in all cases highlighted) indicating either that the incident wave is maintained for a time by diabatic processes operating within the proto-vortex (note that the vortex remains in the trough of the wave for a few days in each of the examples shown) or that new waves are excited by the vortex as it develops into a full-fledged tropical storm, and these waves subsequently propagate as a group to the east. In order to understand the last feature it proves necessary to examine the wave/vortex structure in four dimensions. Most aspects of the subsequent "wave radiation" - if this is the correct way to think of it - are not relevant to 

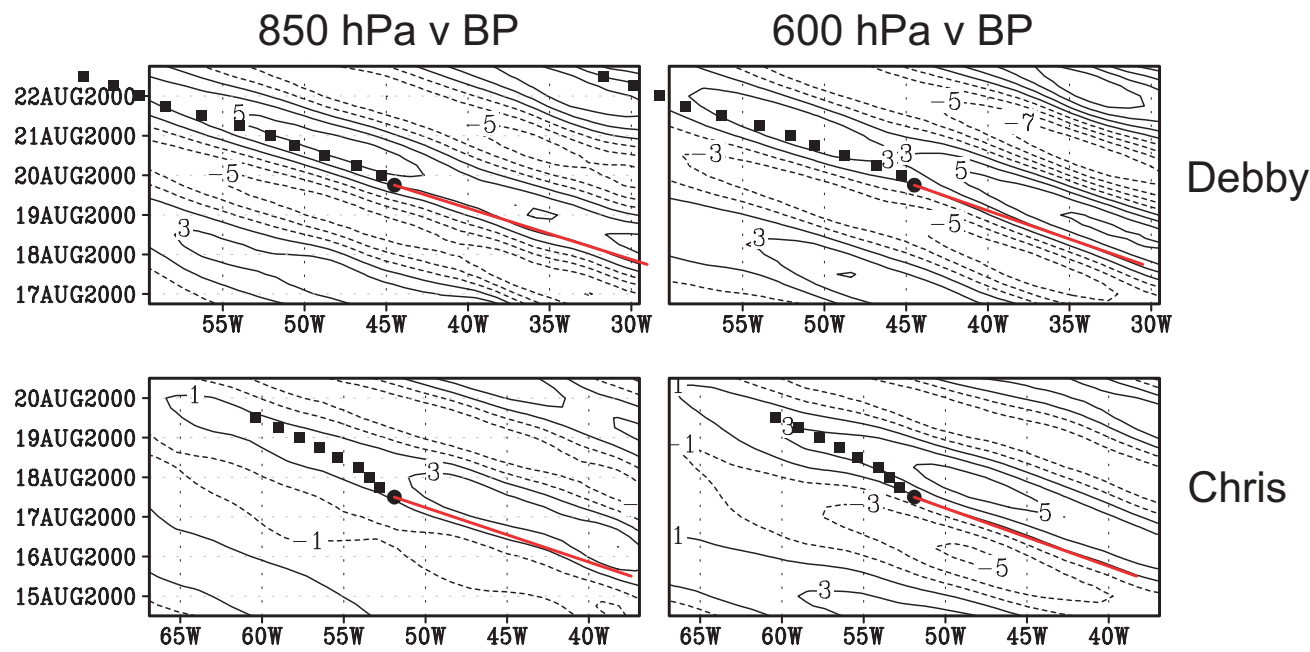

\section{Chris}
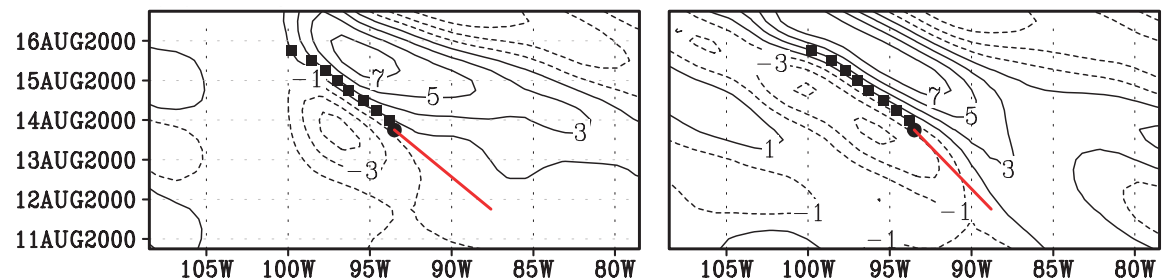

Beryl
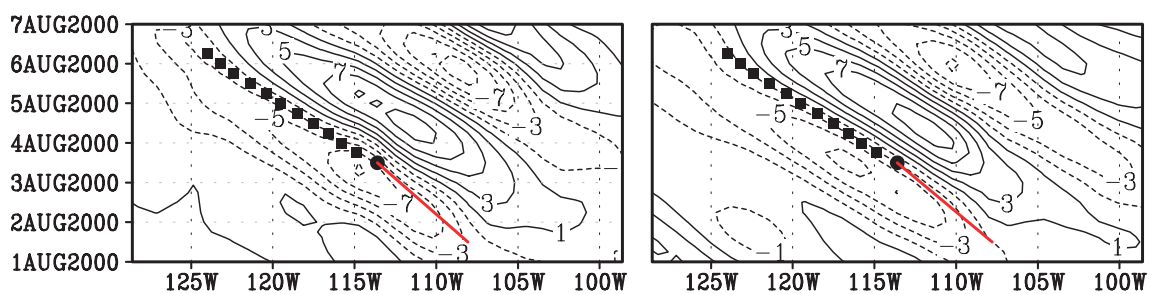

\section{Fabio}
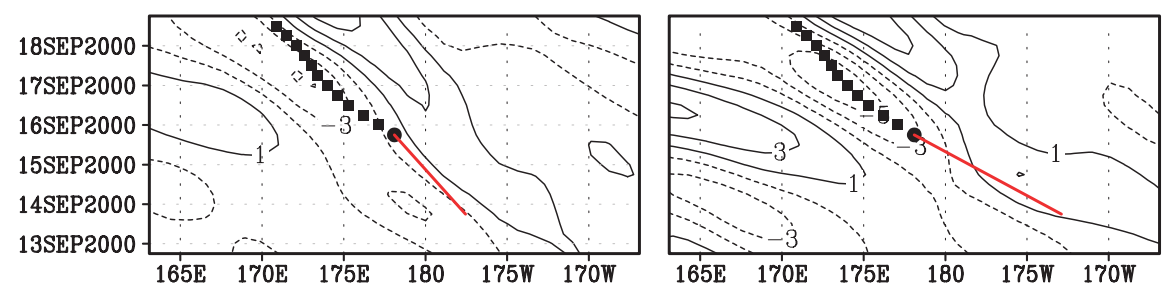

Shanshan

Fig. 4. Hovmöller plots of band-pass meridional velocity component at $850 \mathrm{hPa}$ (left column) and $600 \mathrm{hPa}$ (right column) in a longitude-time plane for the five cases of summer 2000 highlighted in the text: (a) Hurricane Debby; (b) Tropical Storm Chris; (c) Tropical Storm Beryl; (d) Tropical Storm Fabio; (e) Super Typhoon Shanshan. Black circles indicate the best-track longitude and time of genesis, while black squares indicate subsequent best track locations at 6-h intervals. Red line denotes the Hovmöller estimate of zonal translation speed $C_{p}$.

genesis and will be discussed elsewhere. On the other hand, the transition of wave structure from equivalent barotropic in the LT to a deep quadrupole ${ }^{19}$ or "first baroclinic mode" pattern spanning the depth of the troposphere in the longitudeheight plane (along the genesis latitude, at nearby longitudes)

\footnotetext{
${ }^{19}$ A quadrupole structure describes the appearance of meridional velocity anomalies in a longitude-height plane intersecting the vertical axis through a developing storm, with cyclonic (anticyclonic) flow at lower (upper) levels.
}

represents a key signature of genesis process insofar as this structural transition indicates deep convective mass transport coupled to the incident wave and generally precedes the besttrack genesis time by a day or more. This transition will be discussed further in Sect. 4.3. A first baroclinic mode structure was found to be dominant in the western Pacific during boreal summer (Dunkerton, 1993) and probably characterizes the tropical depression disturbances that populate this region. It is noteworthy that a similar structure appears in basins marginal for development (e.g., Atlantic and eastern 
Pacific) just prior to TC genesis. The quadrupole pattern is apparently responsible for the subsequent radiation of a Rossby-like wave which, to our knowledge, has not been discussed previously in the literature but may have important consequences for the UT/LS.

A monochromatic wave, with discrete ground-based frequency and zonal wavenumber, would necessarily look like Fig. $4 \mathrm{a}, \mathrm{b} \ldots$ from which it is obvious that most incident waves are not perfectly monochromatic and therefore contain a modest spread of wavenumbers and frequencies in spectral space. The zonal-wavenumber bandwidth $(\Delta k)$ of incident waves can be estimated from Heisenberg's Uncertainty Principle $\Delta k \Delta x \sim 2 \pi$ or equivalently $\Delta k / k \sim 1 / N$ where $N$ is the number of full cycles observed in the wave packet in the $x$-direction. A similar estimate applies to frequency bandwidth using the number of full cycles in the $t$ direction. Judging from Fig. 4 one might infer a typical value $N \sim 1-3$. Easterly waves, like most tropical waves, are local and episodic in physical space (Dunkerton, 1991). The term "intermittency" (Mandelbrot, 1974) has been used in recent years to describe (i) waves that come and go, having peak quadratic fluxes (of momentum, heat or constituents) much larger than their time-averaged value (Alexander and Dunkerton, 1999); or (ii) turbulence that is inhomogeneous in space or time, containing coherent structures that disrupt the self-similar character of the inertial subrange (Frisch, 1996). As noted in the Introduction, we believe it is necessary to invoke wave and turbulence concepts in order to understand tropical cyclogenesis. In the real world, nonetheless, there is usually a natural order to genesis such that waves precede turbulence in a logical and chronological sense. Since we are considering many wave/vortex events as individual events, not as part of a time-averaged spectrum of geophysical turbulence, it would be better to say that "waves precede vortices." One could then regard the proto-vortex and resulting storm as a coherent structure arising from the wave and interacting with it for a time before acquiring a separate life of its own. The typical sequence of events will become evident in the examples highlighted.

\subsubsection{Central Atlantic: hurricane Debby}

The sloping lines in Fig. 4 represent the mean phase speed derived from the linear regressions of the southerly (solid lines) and northerly (dashed lines) meridional wind anomalies, as described in Appendix A3. The Hovmöller diagrams for Debby, shown in Fig. 4a, b, suggest that the wave has an equivalent barotropic structure in the lower troposphere, as noted in the preceding discussion; the difference of the phase speeds at $850 \mathrm{hPa}$ and $600 \mathrm{hPa}$ is less than $1 \mathrm{~ms}^{-1}$. The similarity of phase speeds is consistent with the barotropic structure evident over the entire diagram.

Figures 5 and 6 show the relative vorticity (representing time scales longer than $\sim 2.5$ days) at the 850 and $600 \mathrm{hPa}$ levels at $6 \mathrm{~h}$ intervals, before the genesis of Debby, from
12:00 Z 18 August to 18:00 Z 19 August 2000. As discussed in Sect. 2, the Lagrangian mean flow in the critical layer is zero in a frame of reference moving at the phase speed of the wave (Fig. 1). Alternatively, the streamlines of a steady vortex are advected by the basic flow (Fig. 3). To illustrate motions relative to the wave and its associated proto-vortex, the streamlines of 2.5-day low pass flow in a "wave-centric" co-moving frame of reference are also shown.

At $850 \mathrm{hPa}$, the best-track genesis location for Debby coincides almost perfectly with the center of a closed gyre in the co-moving frame. The thick curve passing through the center of the gyre denotes the isopleth of zero zonal velocity in the co-moving frame. Along this curve the translating streamlines are oriented meridionally by definition. The thick curve is bracketed by two adjacent curves representing the uncertainty in our phase speed estimate. Adjacent to the center of the gyre, approximately $800 \mathrm{~km}$ to the ENE, is a saddle point or "hyperbolic point" of the translating streamlines. A dividing streamline intersecting the saddle point can be imagined to surround the genesis point, spanning a distance of about $700 \mathrm{~km}$ north to south. Streamlines passing near the saddle point are shown in red. Owing to a small but nonzero divergent component of horizontal motion, the dividing streamline is not exactly closed, but converges towards the center of the gyre in the latter part of the sequence. Significant cyclonic vorticity exists not only within the closed gyre but in a wider area to the south. This additional region of vorticity is less associated with the gyre (as curvature vorticity) than with the jet itself (as shear vorticity). We return to this point in the next example when discussing an alternative measure of vortical flow, the so-called Okubo-Weiss parameter.

At $600 \mathrm{hPa}$, the best-track genesis location likewise coincides with the center of a closed gyre which is much larger in horizontal extent than the gyre at $850 \mathrm{hPa}$. Once again the principal saddle point is located about $800 \mathrm{~km}$ to the east. A secondary saddle point is seen at both levels about $1400 \mathrm{~km}$ to the NW. Three gyres arrayed along an approximately zonal axis from WNW to ESE can be imagined to comprise a series of cat's eyes along the critical latitude for this wave. Strictly speaking, the thick curve in both figures denotes the effective center of the critical layer, not the critical latitude of the wave itself. Supposing that a mean flow as seen by the wave should be represented by a modified low-pass filter (periods $>9$ days) excluding the period of the wave itself (3-4 days), we find that a critical latitude - defined as the locus of points where this low-frequency zonal wind matches the zonal phase speed of the wave - again intersects each of the gyres arrayed zonally in Figs. 5 and 6 (last panel of each). In other words, the critical layer has formed more or less at the critical latitude of the incident wave, as expected from linear theory. This is useful knowledge for wave dynamics but we caution that what actually matters for mesoscale developments is the critical layer with its Kelvin cat's eye, and their subsequent role in TC genesis. 


$$
\text { Debby: Zeta (2.5-day LP) }
$$
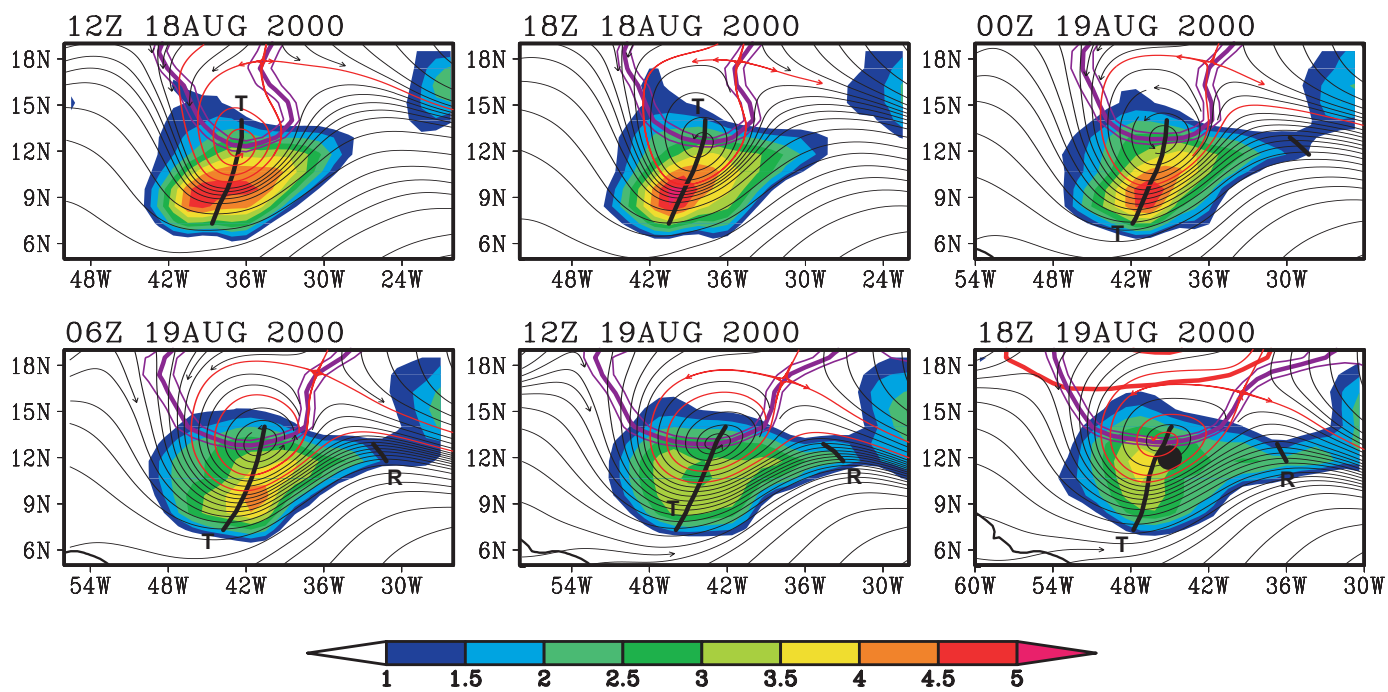

Fig. 5. Streamlines of horizontal (rotational + divergent) flow at $850 \mathrm{hPa}$, as seen in ERA-40 data, for six consecutive analyses leading up to the best-track genesis time of Hurricane Debby (2000). Shading indicates relative vorticity (units: $10^{-5} \mathrm{~s}^{-1}$ ). The sequence of frames translates westward at the zonal propagation speed of the parent wave at $850 \mathrm{hPa}$ as estimated from the Hovmöller method $(-10.0 \pm 0.6 \mathrm{~ms}-1)$ and streamlines are calculated and displayed in the co-moving frame; note that relative vorticity itself is invariant with respect to the translation. Isopleths of zero relative zonal flow are shown (purple) together with their uncertainty. In the final panel of the sequence the critical latitude of the parent wave is also indicated (red) corresponding to isopleths of zero relative zonal flow in low-frequency data with periods longer than 9 days. The wave's trough axis is shown for reference (black). The genesis location is indicated by the black dot in the final panel.

$$
\text { Debby: Zeta (2.5-day LP) }
$$
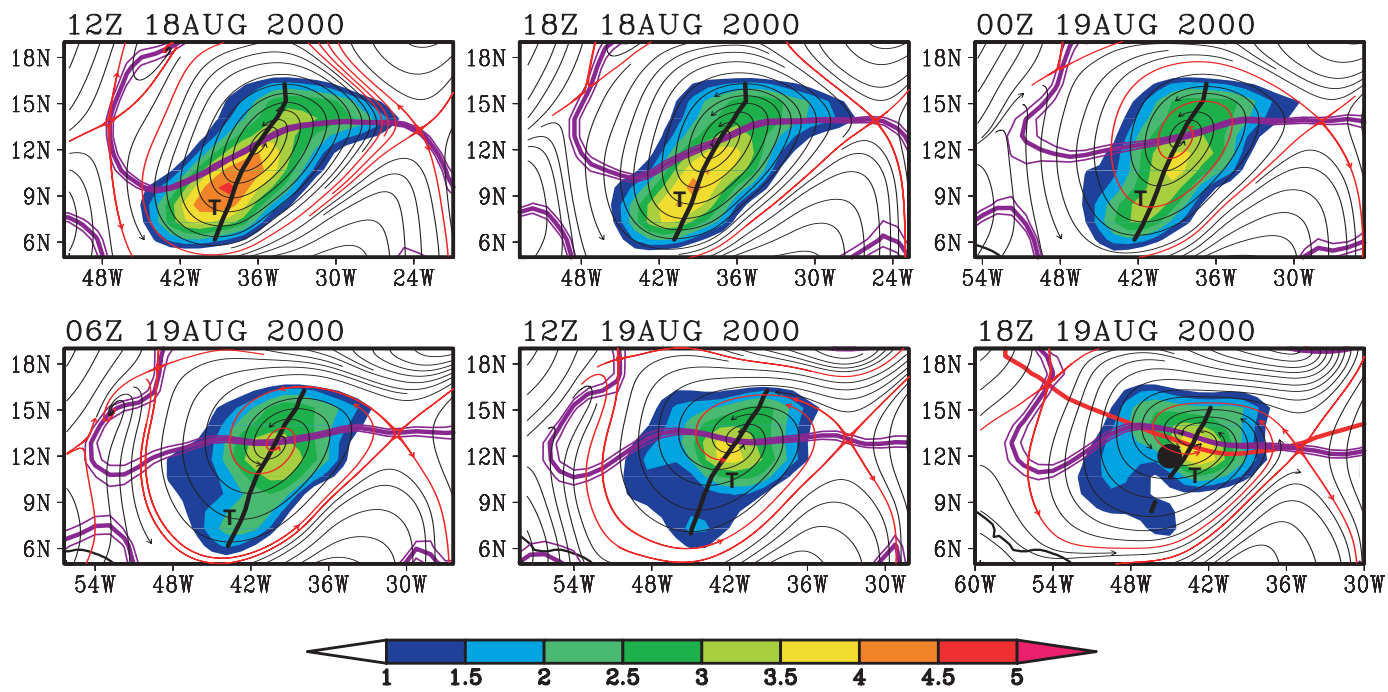

Fig. 6. Streamlines of horizontal flow for the genesis sequence of Hurricane Debby as in Fig. 5, but at $600 \mathrm{hPa}$. Shading indicates relative vorticity (units: $10^{-5} \mathrm{~s}^{-1}$ ). The sequence of frames translates westward at the zonal propagation speed of the parent wave at $600 \mathrm{hPa}$ $\left(-9.1 \pm 0.3 \mathrm{~ms}^{-1}\right)$ and streamlines are calculated and displayed in the co-moving frame.

The importance of the co-moving frame and its specification of "gauge" for flow potentials (Appendix A6) becomes clear when comparing the translating streamlines to those of a resting frame. Figures $7 \mathrm{a}, \mathrm{b}$ illustrate the latter at genesis time only. At $850 \mathrm{hPa}$ there is a closed gyre with principal saddle point misplaced to the $\mathrm{SW}$ of the genesis point by 


\section{Debby: Zeta (2.5-day LP, resting)}

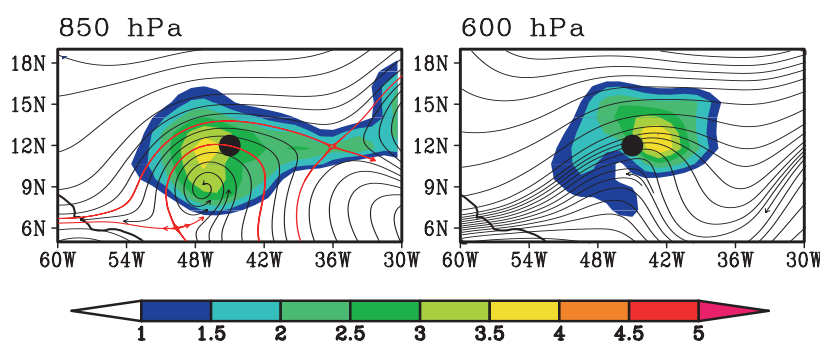

Fig. 7. Streamlines of horizontal flow at (a) $850 \mathrm{hPa}$ and (b) $600 \mathrm{hPa}$ at the genesis time of Hurricane Debby, in the resting frame. Shading indicates relative vorticity (units: $10^{-5} \mathrm{~s}^{-1}$ ) which is invariant with respect to translation, therefore identical in co-moving and resting frames (cp. final panels of Figs. 5, 6).

about $700 \mathrm{~km}$. This pattern seems to have little relation to the distribution of vorticity, even though the choice of gauge in this instance (zero everywhere) is - like any other choice - completely consistent with the vorticity itself. In the Earthrelative frame a substantial part of the motion exists simply to translate the vorticity pattern westward without changing its shape, thereby obscuring the distributive effect of motions within the critical layer. In the translating frame streamlines and isopleths of vorticity are approximately parallel while curving around the southern boundary of the gyre (not unlike the ideal nonlinear critical layer of Fig. 1). At $600 \mathrm{hPa}$ in the resting frame there is no closed gyre at all. Although we do not claim that the choice of translation speed and resulting streamlines in Figs. 5, 6 provide an exact picture of the flow kinematics in this case, the translating frame is clearly superior to the resting frame from a vorticity advection point of view ${ }^{20}$. The near perfect coincidence of a closed gyre in the translating frame with the actual location of TC genesis is also striking. In the resting frame they are far apart. Debby is by no means unique in this respect. We find an actual or probable closed gyre in the translating frame surrounding the genesis point in almost all of the cases in Table 1 (see Sect. 3.3). Note that an optimum overlap of streamlines and vorticity isopleths maximizes Kelvin's circulation around the translating gyre as calculated along the bounding streamline.

Streamlines in the co-moving frame plausibly explain the distribution of moisture in the free troposphere above the

\footnotetext{
${ }^{20}$ For nondivergent barotropic flow the horizontal advection of vertical vorticity is described by the Jacobian of stream function and its Laplacian (all quantities and operators defined in a horizontal plane); translation of the coordinate system with the wave minimizes this quantity by eliminating unsteadiness due to wave propagation. Vorticity advection as viewed in the resting frame primarily causes the pattern to propagate in the direction of the wave. It is desirable to eliminate (via translation) this dominant contribution to the tendency in order to expose more clearly the evolution of the synoptic flow pattern and mesoscale features embedded therein.
}

boundary layer (as represented by the saturation fraction ${ }^{21}$ averaged from the surface to $500 \mathrm{hPa}$, shown in Fig. 8) and of deep moist convection (as represented by the TRMM 3hourly accumulated precipitation, shown in Fig. 9). Their role is "plausible" in the sense that moisture and persistent deep moist convection are located within the translating gyre; a specific role for entrainment of moisture into the developing gyre is less certain (in this case, at least). It is clear from the evolution of saturation fraction that horizontal advection alone cannot explain its distribution. While advection might account, in part, for the wrapping of moist air around the northern side of the gyre, and its transport eastward out of the domain, the entire moistened area in the southern half of the gyre creeps steadily westward against the prevailing flow (with the wave) as if to suggest that the precipitation cells in this region (Fig. 9) are supplying moisture to the gyre. A symbiotic relation between convective moistening and closed gyre circulation evidently exists in this sequence. Convection moistens the free troposphere, the moisture is subsequently contained by the gyre, which favors further deep convection in the gyre. Seeing how some of this moisture is able to escape to the east, we regard the closed circulation as critically important to depression development to the same extent that moist thermodynamic processes are important in the gyre. Progressive saturation of the column (in a singlecolumn point of view) is arguably important to genesis (Raymond and Sessions, 2007; Emanuel, 2007). Our description of the approximately two-dimensional, equivalent barotropic flow kinematics and dynamics in the co-moving frame adds a critical (no pun intended) dimension to the single-column model (SCM) viewpoint while preserving the essential element of deep convective moistening and its consequences. Without the quasi-2-D kinematics and dynamics, the SCM viewpoint has no context within which to explain tropical cyclogenesis.

\subsubsection{East Pacific: tropical storm Fabio}

The evolution of vorticity and translating streamlines in the development of Fabio is shown in Fig. 10 at $850 \mathrm{hPa}$, while Fig. 11 shows the corresponding pattern at $600 \mathrm{hPa}$. In the latter, an alternative measure of vortical flow is provided by the Okubo-Weiss parameter ${ }^{22}$

\footnotetext{
${ }^{21}$ Saturation fraction represents how much water vapor is in the column relative to the maximum that the column might hold, given the observed temperature as a function of pressure (Raymond et al., 2006). A similar quantity was defined by Bretherton et al. (2004). It is equivalent to the vertical integral of relative humidity weighted by saturation mixing ratio.

${ }^{22}$ In geosciences the Okubo-Weiss parameter is used frequently in physical oceanography and two-dimensional turbulence; a few atmospheric applications have been reported (Lukovich and Shepherd, 2005; Rozoff et al., 2006). An improved definition of the parameter for transient flow was developed by Patrice Klein and co-workers (Lapeyre, 2000).
} 


$$
\text { Debby: SF (2.5-day LP) }
$$
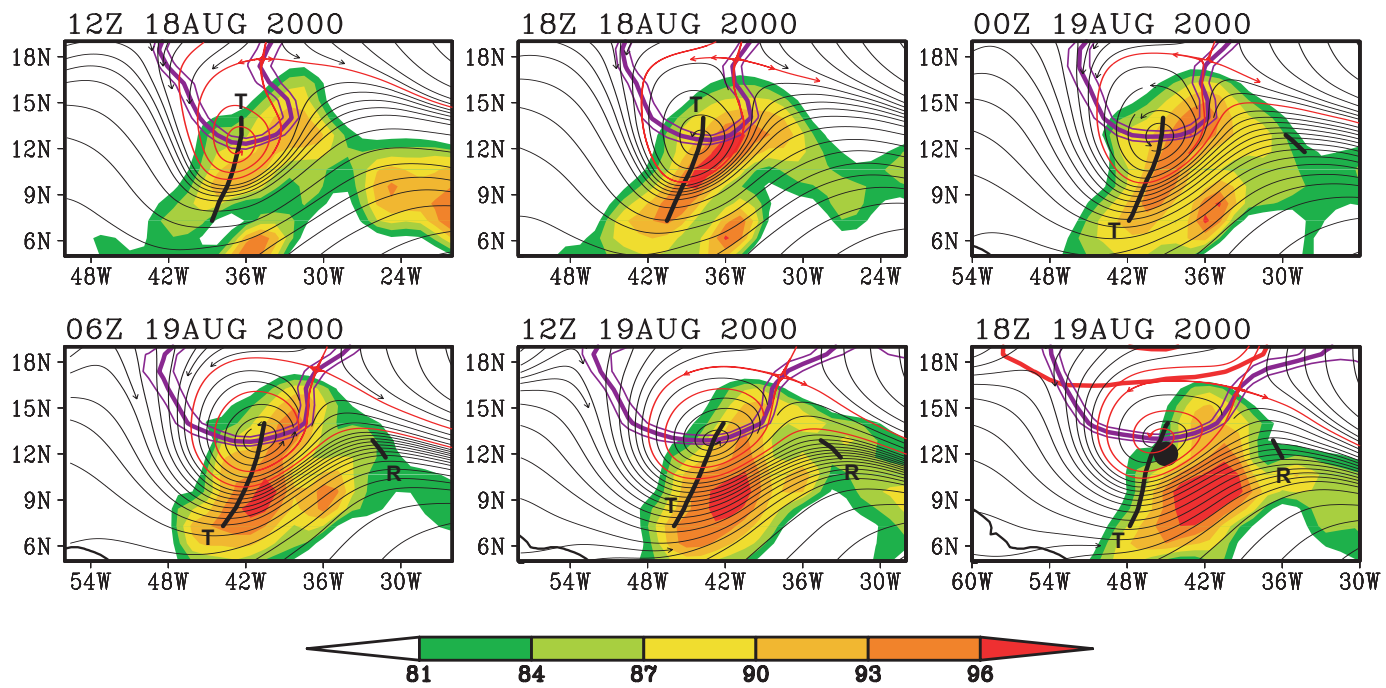

Fig. 8. Streamlines of horizontal flow at $850 \mathrm{hPa}$ for the genesis sequence of Hurricane Debby as in Fig. 5, with high values of ERA-40 saturation fraction indicated by shading (units: percent). The sequence of frames translates westward at the zonal propagation speed of the parent wave at $850 \mathrm{hPa}\left(-10.0 \pm 0.6 \mathrm{~ms}^{-1}\right)$ and streamlines are calculated and displayed in the co-moving frame.

$$
\text { Debby: TRMM (2.5-day LP) }
$$
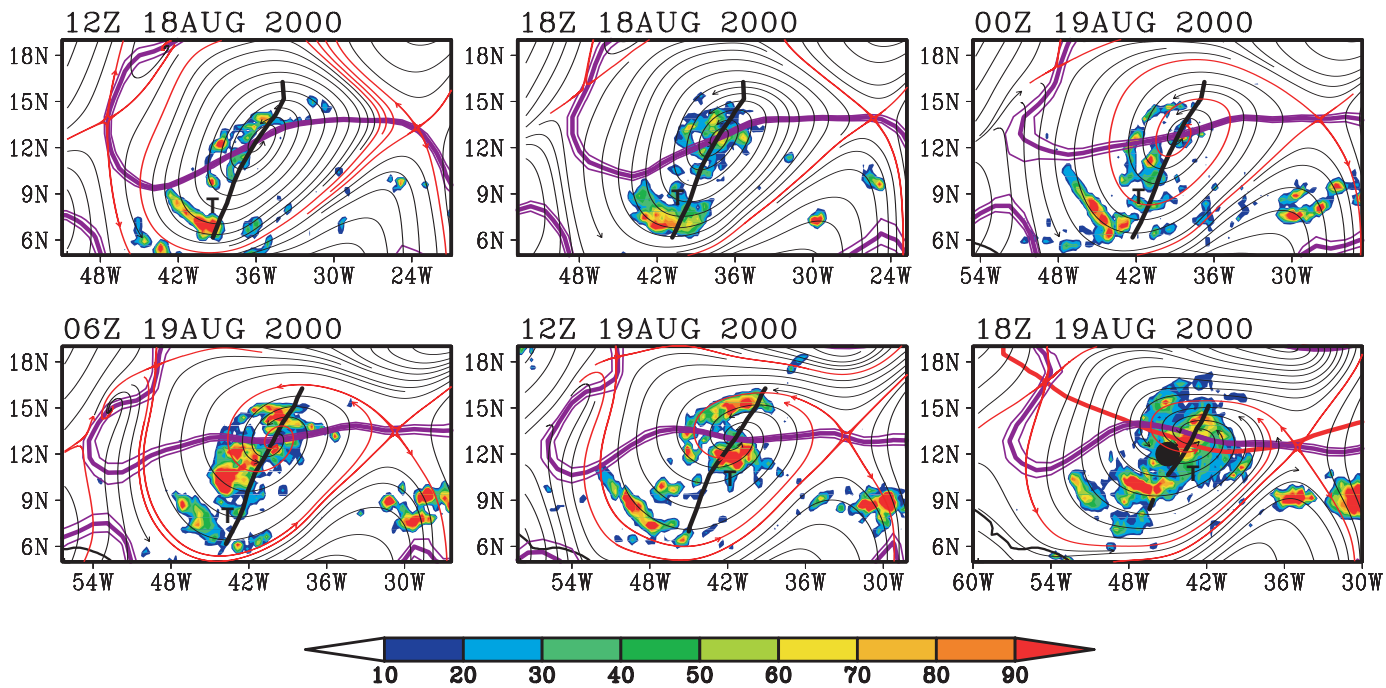

Fig. 9. Streamlines of horizontal flow at $600 \mathrm{hPa}$ for the genesis sequence of Hurricane Debby as in Fig. 6, with high values of TRMM 3-h accumulated precipitation indicated by shading (units: $\mathrm{mm} /$ day). The sequence of frames translates westward at the zonal propagation speed of the parent wave at $600 \mathrm{hPa}\left(-9.1 \pm 0.3 \mathrm{~ms}^{-1}\right)$ and streamlines are calculated and displayed in the co-moving frame.

$$
O W=\varsigma^{2}-S_{1}^{2}-S_{2}^{2}=\left(V_{x}-U_{y}\right)^{2}-\left(U_{x}-V_{y}\right)^{2}-\left(V_{x}+U_{y}\right)^{2}
$$

Patterns of saturation fraction, precipitation and vertical shear in the lower and upper troposphere for this event are shown in Figs. 12-15, respectively. At both levels, closed streamlines formed near the intersection of the critical latitude and the trough axis, delineating a critical layer of fi- nite width. Strong cyclonic/rotational vorticity, high saturation fraction and weak vertical shear coincide simultaneously within the critical layer and provide a favorable sub-synoptic scale environment for tropical storm formation. TRMM data suggests that convective activity is present near the critical latitude $30 \mathrm{~h}$ before genesis was declared by the forecasters. Near the critical latitude the convective activity becomes 
Fabio: Zeta (2.5-day LP)
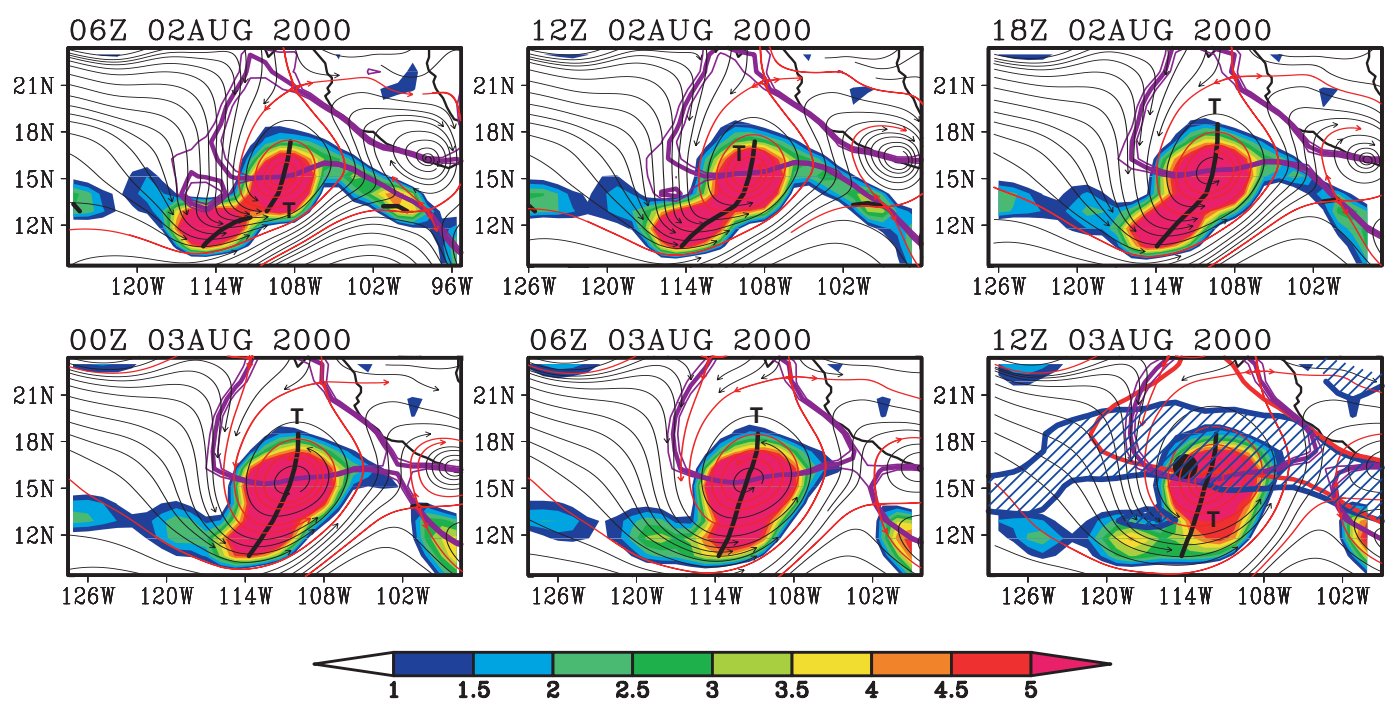

Fig. 10. Streamlines of horizontal (rotational + divergent) flow at $850 \mathrm{hPa}$, as seen in ERA-40 data, for six consecutive analyses leading up to the best-track genesis time of Tropical Storm Fabio (2000). Shading indicates relative vorticity (units: $10^{-5} \mathrm{~s}^{-1}$ ). The sequence of frames translates westward at the zonal propagation speed of the parent wave at $850 \mathrm{hPa}$ as estimated from the Hovmöller method $\left(-3.7 \pm 0.5 \mathrm{~ms}^{-1}\right)$ and streamlines are calculated and displayed in the co-moving frame; note that relative vorticity itself is invariant with respect to the translation. Isopleths of zero relative zonal flow are shown (purple) together with their uncertainty. In the final panel of the sequence the critical latitude of the parent wave is also indicated (red) corresponding to isopleths of zero relative zonal flow in low-frequency data with periods longer than 9 days. These data were also used to estimate the zero contour of absolute vorticity gradient or "effective $\beta$ " (blue). This quantity is negative in the zone just north of the genesis latitude, bracketed by the blue curves (diagonal hatching). The wave's trough axis is shown for reference (black). The genesis location is indicated by the black dot in the final panel.

$$
\text { Fabio: OW (2.5-day LP) }
$$
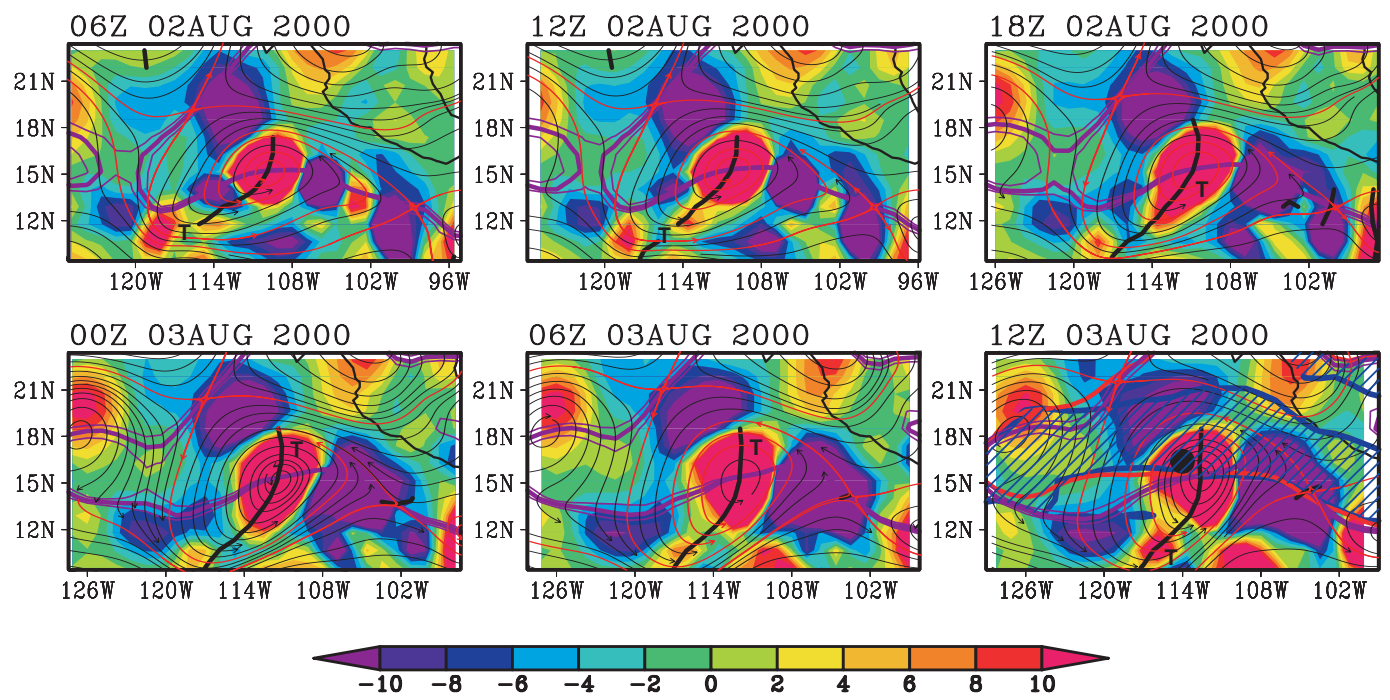

Fig. 11. Stream function of horizontal flow for the genesis sequence of Tropical Storm Fabio as in Fig. 10, but at $600 \mathrm{hPa}$. Shading indicates the Okubo-Weiss parameter (units: $10^{-10} \mathrm{~s}^{-2}$ ) as defined in Eq. (2). This quantity, like vorticity, is invariant with respect to translation, therefore identical in co-moving and resting frames. The sequence of frames translates westward at the zonal propagation speed of the parent wave at $600 \mathrm{hPa}\left(-3.1 \pm 0.8 \mathrm{~ms}^{-1}\right)$ and stream function is calculated and displayed in the co-moving frame. Red and blue lines in the final panel show the wave's critical latitude and isopleth of zero effective $\beta$ as estimated from low-frequency data with periods longer than 9 days. 


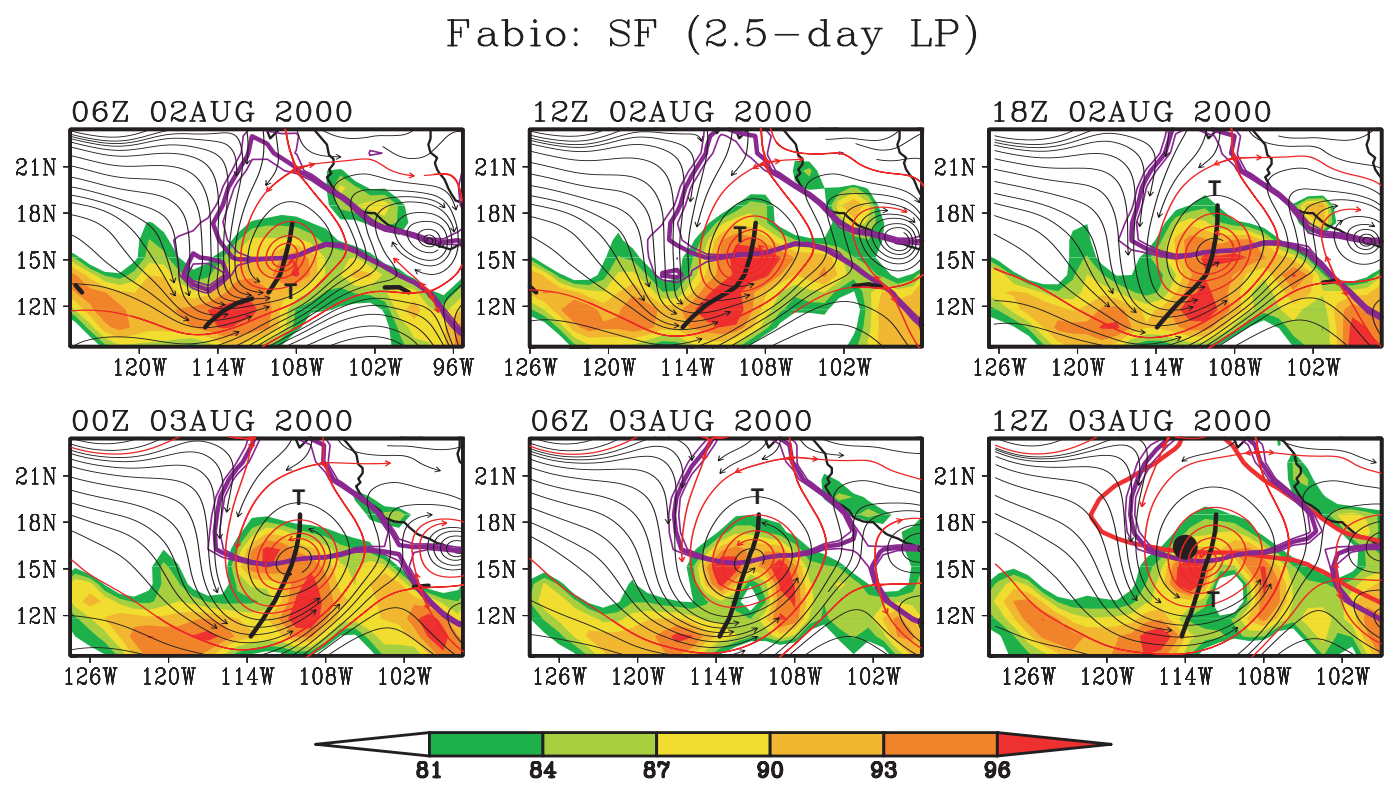

Fig. 12. Streamlines of horizontal flow at $850 \mathrm{hPa}$ for the genesis sequence of Tropical Storm Fabio as in Fig. 10, with high values of ERA-40 saturation fraction indicated by shading (units: percent). The sequence of frames translates westward at the zonal propagation speed of the parent wave at $850 \mathrm{hPa}\left(-3.7 \pm 0.5 \mathrm{~ms}^{-1}\right)$ and streamlines are calculated and displayed in the co-moving frame.

$$
\text { Fabio: TRMM (2.5-day LP) }
$$
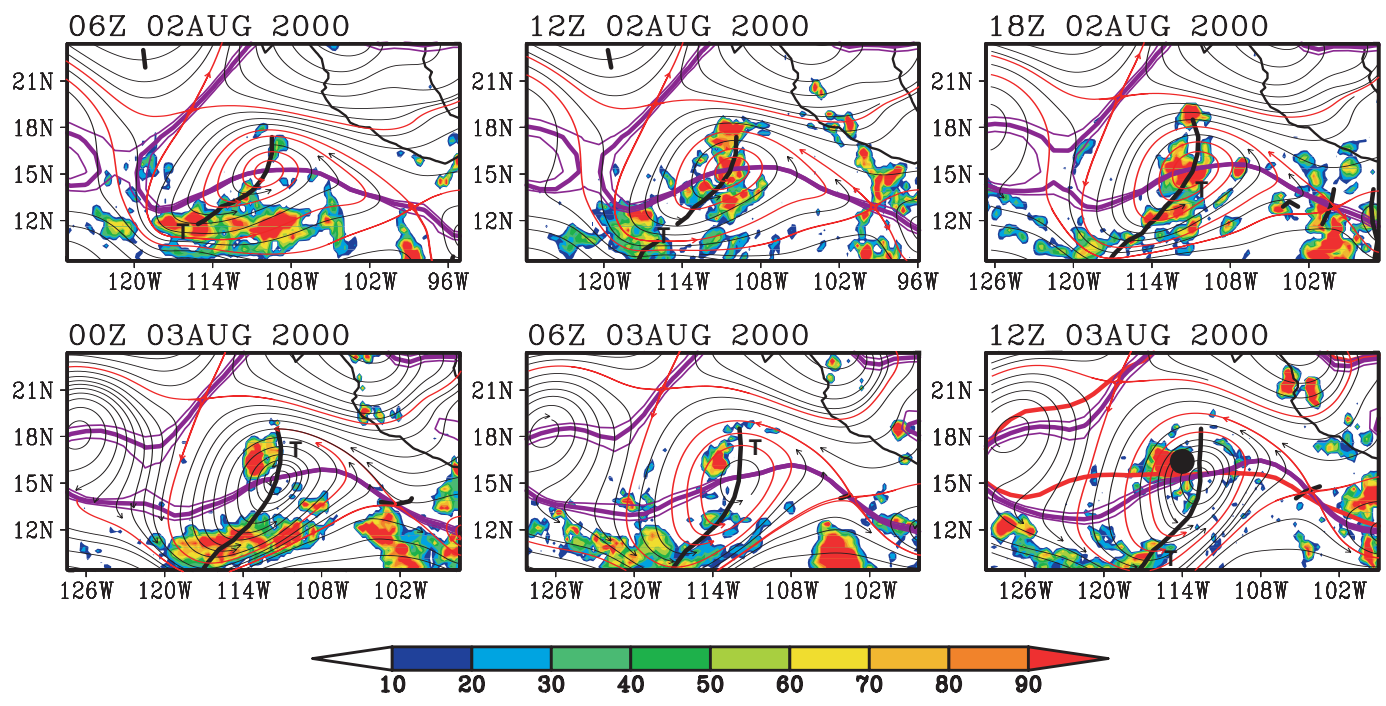

Fig. 13. Streamlines of horizontal flow at $600 \mathrm{hPa}$ for the genesis sequence of Tropical Storm Fabio as in Fig. 11, with high values of TRMM 3-h accumulated precipitation indicated by shading (units: $\mathrm{mm} /$ day). The sequence of frames translates westward at the zonal propagation speed of the parent wave at $600 \mathrm{hPa}\left(-3.1 \pm 0.8 \mathrm{~ms}^{-1}\right)$ and streamlines are calculated and displayed in the co-moving frame.

sustained, and more focused, while the convective activity away from the local critical latitude dies out. It is striking that genesis eventually occurs very close to the intersection of the trough axis and the critical latitude (as true for the other cases highlighted). Also striking is that the eventual location of TC genesis lies just within a wet zone delineated by a strong north-south gradient of moisture, as depicted by the color shading in Fig. 12. This configuration is expected in cases where genesis occurs poleward of, but close to, the ITCZ. Expansion of the closed gyre during the pre-genesis sequence exaggerates the weak undulation of moisture ribbon that was present at the start. With a fully developed 
hurricane nearby, the amplified pattern indicates a severe deformation or demise of the original ITCZ (Dunkerton, 2006) although this is not yet apparent in the final panel of Fig. 12 . By this time, however, deep convection has become notably absent in the diffluent region (lower right corner) in the final panel of Fig. 13. Farther east, deep convection has become invigorated in a second closed gyre.

The genesis of Fabio, over the eastern Pacific, may be more closely related to the ITCZ than the other storms highlighted thus far in the sense that cyclonic vorticity, convection and high humidity all have a zonally elongated pattern prior to genesis. The actual storm, however, is located north of the ITCZ, and is associated with a break in the zonally elongated pattern. As noted by Dunkerton (2006), it is common for tropical storms to displace poleward or to obliterate the ambient ITCZ. The role of the ITCZ in such cases is not obvious. Although hydrodynamic instability yields a similar outcome, as instabilities become nonlinear and detached from their source (viz., an unstable strip of PV along the original ITCZ: see, e.g., Fig. 5 of Wang and Magnusdottir, 2005) it is plausible that neutral easterly waves propagating parallel to the ITCZ have a similar effect in cases where genesis ensues. In Fabio, the easterly wave exists ahead of time (as shown by the Hovmöller plots) and the original critical latitude and isopleth of zero PV gradient (blue curve) were nearly coincident. It appears that the wave prior to genesis was nearly neutral (according to the Rayleigh criterion) or at most, marginally unstable ${ }^{23}$.

A final point is that the best-track genesis location does not coincide with the minimum vertical shear as measured by either of our two metrics (lower to mid- or upper troposphere, as shown in Figs. 14-15, respectively). It is clear from this case and others, and from our modeling study, that the horizontal distribution of vertical shear is not the deciding factor in where a storm will form. As will be shown later, shears of this magnitude do not prevent development, so it is not surprising that Fabio's origin does not coincide with the location of minimum shear. Of more importance is that vertical shears in the neighborhood of the genesis point are not extremely large (much greater than $10 \mathrm{~ms}^{-1}$ ). It should be noted that vertical shear over the depth of the troposphere is influenced by deep moist convection and unrelated synoptic activity in the UT, as discussed in Sect. 4, not by easterly waves alone.

Maps for the three additional highlighted cases are described in Appendix B: Chris (noted for early detection of closed streamlines in the co-moving frame prior to genesis), Beryl (noted for interaction of easterly wave and frontal boundary) and Shanshan (noted for its evolving vertical wave phase tilt).

\footnotetext{
${ }^{23}$ The marsupial paradigm embraces the hydrodynamically unstable pathway insofar as the critical layer (relevant to TC formation) is an essential part of the synoptic-scale instability itself, as noted above.
}

\subsection{Survey of events 1998-2001}

Our diagnoses of the 55 named storms in Table 1 and the five cases highlighted herein support the hypotheses (H1H2) that the critical layer containing the surface low of the parent wave, and Kelvin cat's eye within, is a preferred region or sweet spot for TC genesis. The intersection of (eastwest) critical latitude and (north-south) wave trough corresponds to the center of the Kelvin cat's eye of nonlinear critical layer theory (Fig. 1). Such features correspond to regions of closed Lagrangian circulation which, on the southern side of the AEJ axis, have cyclonic vorticity and abundant moisture.

An obvious closed gyre in ERA-40 data is found at $600 \mathrm{hPa}$ in the translating frame within $30 \mathrm{~h}$ prior to genesis in 53 of the 55 cases; in one of the remaining cases (Joyce) the gyre is probable and in another (Dean) possible ${ }^{24}$. At $850 \mathrm{hPa}$ the statistics are almost as good: 50 of 55 are obvious, 1 probable, 3 possible, and 1 unlikely. A closed gyre implies one or more saddle points in the vicinity. Inspection of the 55 selected cases reveals a remarkable result that, whereas this point is usually located to the south of the gyre center in the resting frame, it is almost never located directly to the south in the co-moving frame (Table 1). Meteorological charts displaying Earth-relative streamlines are a standard tool in the forecasting industry, but generally provide a misleading picture of actual flow kinematics, or Lagrangian displacement of air, in propagating tropical waves. These maps typically display an upright omega pattern in the lower troposphere straddling the wave's trough axis. In fact the omega pattern is often upside-down in the translating frame, with separatrix orientation to the north. In other cases, the separatrix is oriented to the east or west (or both). To the extent that horizontal advection explains the topology of streamlines, and therefore indicates where air in the closed gyre has detached from, it is firmly established in our 55 cases that air at $600 \mathrm{hPa}$ is not derived from or being exchanged (by resolvable motions) with distant points to the south. For the most part, this is also true at $850 \mathrm{hPa}$. Rather, it is coming from (or previously associated with) air to the east, north or west of the gyre center. This result is probably the most important take-home message for operational purposes and weather briefings.

\footnotetext{
${ }^{24}$ In Joyce, the estimate of zonal translation speed in Table 1 is exaggerated owing to a northward component of wave propagation that is not accounted for by this analysis; a closed gyre at $850 \mathrm{hPa}$ is found at slower translation speed $\left(-4\right.$ to $-5 \mathrm{~ms}^{-1}$; not shown). In Dean, storm formation occurred at the separatrix of a cutoff anticyclone on the SW side of the Bermuda High, a narrow trough whose kinematic features are partially attenuated by the ERA-40 reanalysis and possibly by our 2.5-day low-pass filter. A northward component of propagation is evident in this event also. All of these challenges (and others not mentioned) make the identification of Lagrangian boundaries more difficult in such cases.
} 


$$
\text { Fabio: UV500-UV850 (2.5-day LP) }
$$
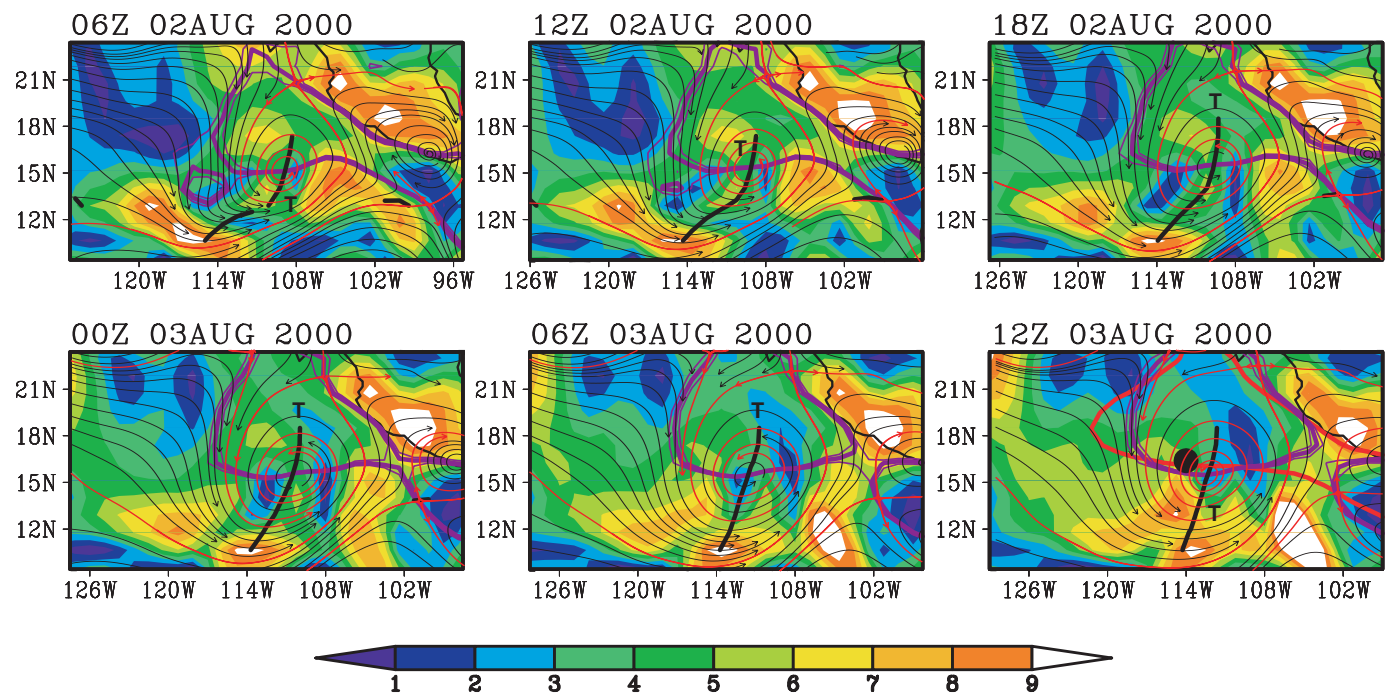

Fig. 14. Streamlines of horizontal flow at $850 \mathrm{hPa}$ for the genesis sequence of Tropical Storm Fabio as in Fig. 10, with ERA-40 850-500 hPa vertical shear (magnitude of shear vector, units: $\mathrm{ms}^{-1}$ ) indicated by shading. The sequence of frames translates westward at the zonal propagation speed of the parent wave at $850 \mathrm{hPa}\left(-3.7 \pm 0.5 \mathrm{~ms}^{-1}\right)$ and streamlines are calculated and displayed in the co-moving frame.

$$
\text { Fabio: UV200-UV850 (2.5-day LP) }
$$
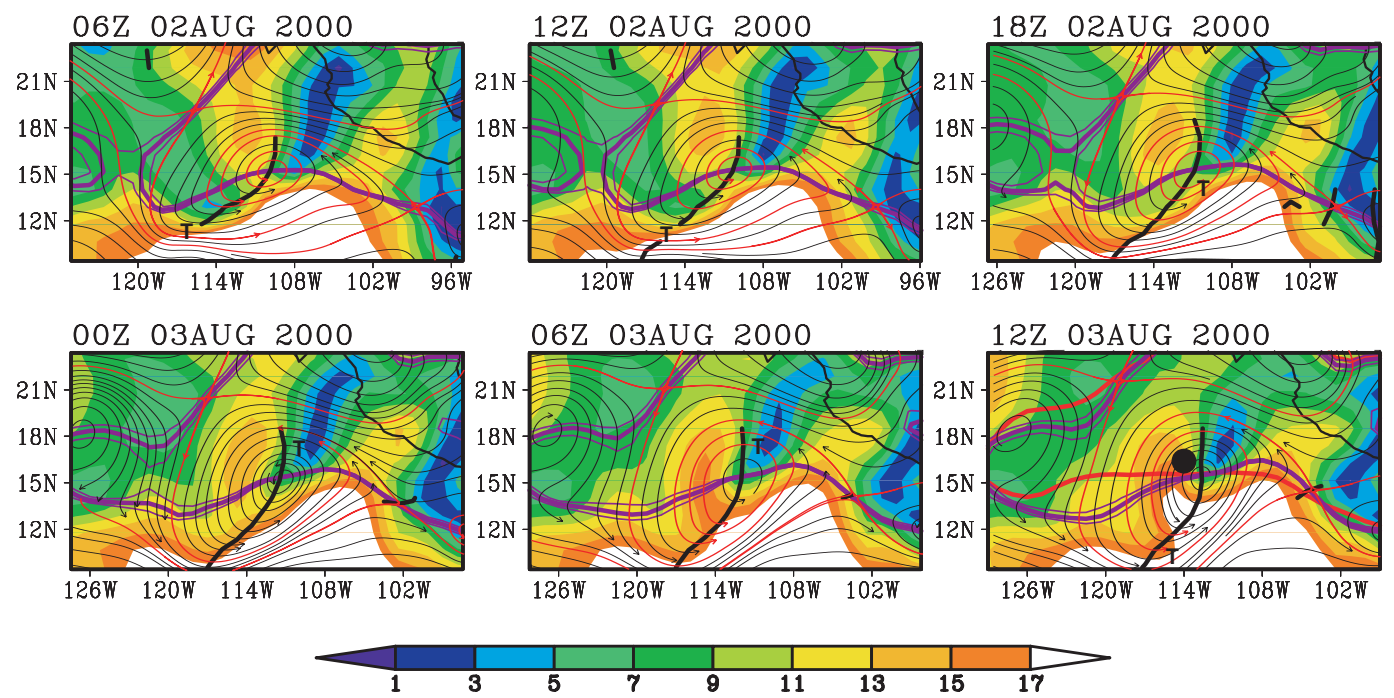

Fig. 15. Streamlines of horizontal flow at $600 \mathrm{hPa}$ for the genesis sequence of Tropical Storm Fabio as in Fig. 11, with ERA-40 850-200 hPa vertical shear (magnitude of shear vector, units: $\mathrm{ms}^{-1}$ ) indicated by shading. The sequence of frames translates westward at the zonal propagation speed of the parent wave at $600 \mathrm{hPa}\left(-3.1 \pm 0.8 \mathrm{~ms}^{-1}\right)$ and streamlines are calculated and displayed in the co-moving frame.

Using the concept of "translating closed gyre" the statistical properties of dynamical fields (e.g., vorticity, OkuboWeiss parameter and vertical shears) and thermodynamical fields (e.g., saturation fraction, deep convective precipitation) relevant to TC genesis can be evaluated within the observed gyres - fields that were illustrated for representative cases in the preceding figures. Rather than display these statistics in obscure tabular form, we summarize the results pictorially in Figs. 16-17 for the 850 and $600 \mathrm{hPa}$ levels, respectively. We focus here on the simplest of all statistics: the mean. Various spatio-temporal averages were constructed by selecting from two choices of spatial average (horizontal expanse of 

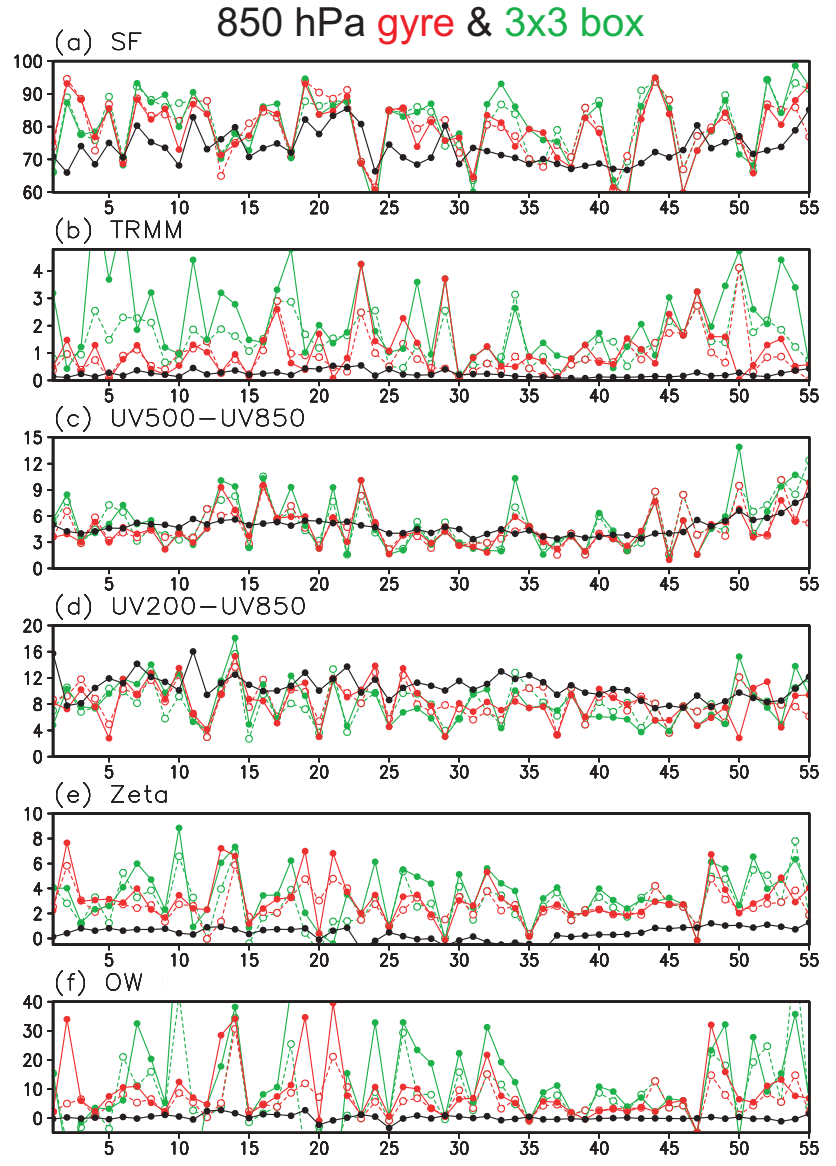

Fig. 16. Spatio-temporal average of dynamical and thermodynamical fields in the neighborhood of the best-track genesis location and time immediately preceding. The storms are arrayed west to east, from left to right, in the reverse of Table 1. The spatial average corresponds either to that of (i) the translating gyre (red) or (ii) a $3 \times 3$ matrix of ERA-40 grid points surrounding the point $x(t)=x_{0}-C_{p} \cdot\left(t-t_{0}\right)$ where $x$ is longitude, $t$ is time, $C_{p}$ is the speed of translation and the subscript 0 refers to the location and time of genesis (green). The temporal average corresponds either to (i) the genesis time (single analysis, filled circles) or (ii) the 36$\mathrm{h}$ interval preceding and slightly overlapping genesis time (average of six consecutive 6-hourly analyses ending at genesis time, open circles). Black circles denote "climatological" values for AugustSeptember 1998-2001 at the various gyre or grid box locations. The data shown in the figure were derived using gyre properties and translation speeds at $850 \mathrm{hPa}$ : (a) saturation fraction averaged from 1000-500 hPa (units: percent), (b) TRMM deep convective precipitation (units: $\mathrm{mm} /$ day), (c) vertical shear from lower to midtroposphere $\left(850\right.$ to $500 \mathrm{hPa}$, units: $\left.\mathrm{ms}^{-1}\right)$, (d) vertical shear from lower to upper troposphere ( 850 to $200 \mathrm{hPa}$, units: $\mathrm{ms}^{-1}$ ), (e) relative vorticity at $850 \mathrm{hPa}$ (units: $10^{-5} \mathrm{~s}^{-1}$ ), (f) Okubo-Weiss parameter at $850 \mathrm{hPa}$ (units: $10^{-10} \mathrm{~s}^{-2}$ ). Please refer to footnote 25 for important information regarding the climatology of vertical shear and Okubo-Weiss. the translating gyre versus a $3 \times 3$ matrix of ERA-40 grid points moving at the wave phase speed and ending at the genesis location) and two choices of temporal average (genesis time versus a 36-h average preceding and slightly overlapping genesis time). To put these average values in context, they are compared to "climatological" values defined simply as the August-September 1998-2001 average at the location of each gyre or $3 \times 3$ array. The climatological values are independent of time by definition, but depend on spatial position; the variety of observed gyres effectively samples the climatology in many different ways, depending on gyre location and extent, and optimum translation speed $C_{p}$. It is important to note that what distinguishes this analysis from all previously published studies of the genesis environment is that we include in our menu of averaging operators a zonal translation of the array of selected points; that is, a spatial array that is a function of time, owing to wave propagation.

Space precludes a detailed discussion of each storm. Apart from a small number of oddballs, the message is clear: the observed storms formed in relatively moist, precipitating environments with large values of vorticity and Okubo-Weiss parameter. This is true for gyre and local averages alike, but the local average contains some exceptionally large values of precipitation and Okubo-Weiss parameter, suggesting that (i) the TRMM satellite has observed enhanced precipitation associated with the developing storm, concentrated at the best-track location rather than distributed throughout the gyre, and (ii) ECMWF analyses have pinpointed the location of the developing vortex within the gyre and identified its vorticity as predominantly rotational rather than deformational, i.e., not deformed by strain or shear. The persistence of TRMM precipitation in the $30+\mathrm{h}$ preceding genesis is evident - the translation of coordinate system is crucial for proper alignment - and the same can be said for relative vorticity and Okubo-Weiss. A null hypothesis for oddball cases (exceptions to the pattern) is that they represent a failure of the analysis to capture the actual circulation or moisture, not a freak development.

Although vertical shear is not necessarily small in developing environments prior to genesis, values are either comparable to (at $850-500 \mathrm{hPa}$ ) or somewhat less than (at 850 $200 \mathrm{hPa}$ ) the climatological shears ${ }^{25}$. The local shear is often smaller than the gyre-wide shear, especially for $600 \mathrm{hPa}$ gyres which can be expansive. This suggests that the center

\footnotetext{
${ }^{25}$ Climatological shears were obtained by first calculating the modulus of the shear vector, then averaging together to form the climatology. In storm development regions, vertical shear of the climatological wind velocity is small $\left(\sim 3 \mathrm{~ms}^{-1}\right.$, not shown). Developing systems generally form in shear that exceeds the "shear of the climatology" but is comparable to or less than the "climatology of the shear". The climatology of Okubo-Weiss parameter was obtained in a similar way, by first calculating the quadratic products and then averaging. Developing systems generally form in environments with Okubo-Weiss parameter well above its climatological range.
} 
of the gyre (or proto-storm location) is already a favorable spot for genesis, relative to the gyre as whole. Note that the average shear as defined here represents the average of a positive definite quantity (modulus of the shear vector at each point within the gyre) and therefore disallows any cancellation of opposing shears in different parts of the gyre. The average indicates, rather, a typical vertical shear that might be seen by convective elements in each grid-box column, regardless of shear vector orientation. Storms are evidently able to form in environments containing a wide range of shear values from $3-12 \mathrm{~ms}^{-1}$. These values admittedly are not huge, and as forecasters are aware, local shears in hostile environments can be much larger, exceeding $20 \mathrm{~ms}^{-1}$. For developing storms, as displayed in Figs. 16-17, vertical shears in the Eulerian frame are comparable to the differences in optimum phase speed $C_{p}$ between the lower and upper troposphere (Sect. 4.3). Neither of these quantities exceed $10 \mathrm{~ms}^{-1}$, except on rare occasions, so it is likely that the Lagrangian shear seen by the developing vortex is small as well. The last remark is intended to expose a point easily overlooked: if a pouch were tilted against a modest shear initially, but made erect by the shear over several days (requiring wave phase speed to vary with height), deep moist convection reaching the upper troposphere in an hour or less could then occur in upright fashion throughout the depth of the pouch, favoring storm development. Larger vertical shear, on the other hand, might stifle vortical organization by shearing the clouds themselves, favoring linear organization.

Once formed, the storm may help to maintain small shear, to the extent that it can. Divergent outflow originating above the storm center ensures that modest vertical shear will continue to exist off-center (even when the environmental shear is negligible) while remaining small on-center. Hendricks and Montgomery (2006) suggested that deep convection associated with vortical hot towers can erode vertical shear, as suggested by Molinari et al. (1995). This mechanism is important in tropical transition, where vertical shear owing to the UT cyclone upstream (to the NW of the developing low-level center) is unfavorable for further development and must be kept at bay (Davis and Bosart, 2004). Similar vulnerability to adverse shear may exist for storms originating in the MDR when "troughy" conditions prevail aloft. In the next section the vertical structure and evolution of waves and their associated critical layer is described in more detail for Hurricane Debby (2000). In this case the divergent outflow becomes visible prior to genesis, and is weakly anticyclonic.

The relation of "wave" (as defined by the band-pass meridional velocity anomaly) and "vortex" (as defined by the besttrack location following genesis) in the 55 storms is summarized compactly in Table 2. After genesis, the average best-track location moves from its initial location near the trough axis (zero phase) to a somewhat negative phase; i.e., east of the westward-moving axis. The drift is much larger in cases where the best track drifts eastward in the Earthrelative frame later in the sequence ("eastward-also"). In
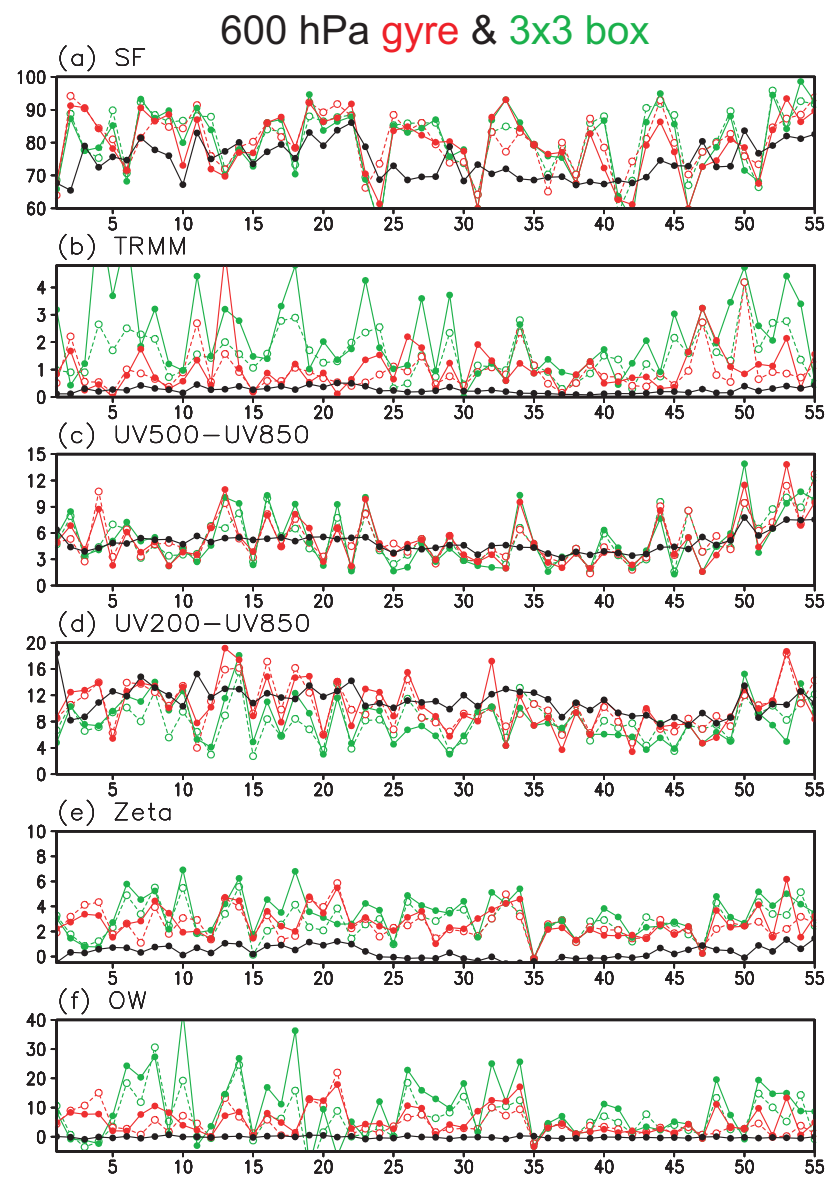

Fig. 17. Spatio-temporal average of dynamical and thermodynamical fields as in Fig. 16, but for $600 \mathrm{hPa}$ gyres and translation speeds. Vorticity and Okubo-Weiss parameter from the 600, rather than $850 \mathrm{hPa}$ level are shown in panels $(\mathbf{e}, \mathbf{f})$.

each category, the relative drift or lack thereof is statistically significant. Average wave amplitude declines following genesis, but the variability among cases is large. A longer time interval following genesis is evidently required in order to witness a significant amplitude decline. Conversely, one could argue that maintenance of wave amplitude during the time period shown supports $\mathrm{H} 3$, although quantitative proof of the hypothesis is beyond the scope of this paper.

\section{Three-dimensional structure and evolution of a tropi- cal wave critical layer}

Two observations are evident from the historical record of TC genesis and early intensification over the Atlantic and eastern Pacific for events having a clear signature of tropical waves prior to genesis. (i) A "surface low along the wave" is a necessary, but not sufficient, condition for genesis of a tropical depression. (ii) In all but a few cases, genesis of a tropical depression is a sufficient condition for further intensification 
Table 2. Peak-to-peak wave amplitude (band-pass meridional velocity anomaly, $\mathrm{ms}^{-1}$ ) from genesis time to the end of the Hovmöller sequence ( +3 days) and variation of vortex phase relative to wave trough (degrees) over this time interval. Negative relative phase is located to the east of the trough. The normalized distance from trough to ridge is $180^{\circ}$ by definition. For wave amplitude (vortex phase) the average (median) and standard deviation (one-half inter-quartile range) of initial and final values are displayed in the first and second rows of each pair, respectively.

\begin{tabular}{|c|c|c|c|c|c|c|c|c|c|c|c|c|}
\hline & \multicolumn{3}{|c|}{$850 \mathrm{hPa}$ wave amplitude } & \multicolumn{3}{|c|}{$600 \mathrm{hPa}$ wave amplitude } & \multicolumn{3}{|c|}{$850 \mathrm{hPa}$ vortex phase } & \multicolumn{3}{|c|}{$600 \mathrm{hPa}$ vortex phase } \\
\hline & initial & final & change & initial & final & change & initial & final & change & initial & final & change \\
\hline \multirow[t]{2}{*}{ all events [55] } & 4.3 & 3.8 & -0.5 & 3.9 & 3.5 & -0.4 & 8 & -8 & -16 & 21 & -21 & -42 \\
\hline & 2.0 & 2.0 & - & 1.7 & 1.5 & - & 25 & 37 & - & 26 & 39 & - \\
\hline \multirow[t]{2}{*}{ all events, Atlantic [31] } & 4.3 & 3.8 & -0.5 & 3.8 & 3.4 & -0.5 & 3 & -30 & -33 & 8 & -26 & -35 \\
\hline & 1.8 & 1.8 & - & 1.5 & 1.5 & - & 20 & 27 & - & 21 & 27 & - \\
\hline \multirow[t]{2}{*}{ all events, E Pacific [23] } & 4.5 & 3.8 & -0.7 & 4.0 & 3.7 & -0.4 & 35 & 21 & -14 & 30 & -15 & -45 \\
\hline & 2.2 & 2.3 & - & 1.9 & 1.6 & - & 29 & 38 & - & 29 & 62 & - \\
\hline \multirow[t]{2}{*}{ wwd only [40] } & 4.7 & 4.1 & -0.7 & 4.1 & 3.8 & -0.3 & 0 & 0 & 0 & 0 & -15 & -15 \\
\hline & 2.1 & 1.9 & - & 1.8 & 1.5 & - & 26 & 33 & - & 23 & 39 & - \\
\hline \multirow[t]{2}{*}{ wwd only, Atlantic [22] } & 4.8 & 4.0 & -0.7 & 4.0 & 3.6 & -0.4 & 0 & -10 & -10 & -4 & -18 & -14 \\
\hline & 1.8 & 2.0 & - & 1.6 & 1.5 & - & 21 & 26 & - & 19 & 28 & - \\
\hline \multirow[t]{2}{*}{ wwd only, E Pacific [17] } & 4.8 & 3.9 & -0.9 & 4.2 & 3.9 & -0.3 & 23 & 21 & -2 & 23 & -7 & -29 \\
\hline & 2.4 & 1.9 & - & 2.0 & 1.7 & - & 27 & 25 & - & 31 & 53 & - \\
\hline \multirow[t]{2}{*}{ ewd also [15] } & 3.3 & 3.3 & -0.1 & 3.4 & 2.7 & -0.7 & 25 & -45 & -70 & 36 & -50 & -86 \\
\hline & 1.2 & 2.2 & - & 1.4 & 1.3 & - & 29 & 26 & - & 20 & 41 & - \\
\hline \multirow[t]{2}{*}{ ewd also, Atlantic [9] } & 3.2 & 3.1 & -0.1 & 3.3 & 2.7 & -0.7 & 18 & -51 & -69 & 36 & -50 & -86 \\
\hline & 1.4 & 1.2 & - & 1.3 & 1.4 & - & 20 & 16 & - & 17 & 41 & - \\
\hline \multirow[t]{2}{*}{ ewd also, E Pacific [6] } & 3.5 & 3.5 & 0.0 & 3.5 & 2.8 & -0.7 & 45 & 15 & -30 & 47 & -65 & -112 \\
\hline & 0.8 & 3.3 & - & 1.5 & 1.2 & - & 35 & 43 & - & 34 & 59 & - \\
\hline
\end{tabular}

to named storm (tropical storm and possibly hurricane). Our definition of "genesis" in Sect. 1 is equivalent to the development of a depression-strength vortex, not to any subsequent intensification. From the results reported above, the development of a critical layer in the lower troposphere is evidently a necessary condition for genesis (and further intensification) by statistical association (Table 1) and by physical reasoning as outlined in the three hypotheses of Sect. 2.2. It is obviously desirable to find conditions, in addition to the existence of a critical layer, that would be sufficient for a tropical depression. Here "sufficient" could mean either that (i) in an ensemble of realizations, a tropical depression forms in most cases, or (ii) in a single realization, such development is guaranteed. The latter definition is more strict and arguably impractical for the chaotic dynamics of geophysical turbulence as it would require observations on all relevant scales, accurate and fine enough to overcome (with predictive capability, too) the uncertainties of vortex aggregation and triggering of deep moist convection (M06; Van Sang et al., 2008; Schecter and Dunkerton, 2007). The relaxed definition of sufficiency opts instead for a statistical measure, e.g. of variances or co-variances of mesoscale fields, when considering possible conditions for up-scale development and formation of a tropical depression.

Unfortunately even a statistical measure is difficult to obtain, as it is impossible to calculate mesoscale (co-)variances either from analysis data, which are too coarse in horizontal resolution, or from field campaigns, which are too brief to provide good statistics. It seems that modeling studies are needed for this purpose (Montgomery et al., 2009). We can, however, dig a little deeper into the available observations to define conditions that come closer to sufficiency for tropical depression formation. In this section we provide additional details that may be relevant for this purpose as well as for quantitative evaluation of the three hypotheses outlined in Sect. 2.2. We provide a partial but incomplete evaluation, for the fundamental reason mentioned above. Our analysis will focus on the kinematics of parcel motions, the vertical structure of the hurricane embryo, and the vertical structure of waves that create its environment. For this purpose our discussion will highlight aspects of Debby (Atlantic 2000) that are broadly typical of the cases listed in Table 1.

\subsection{Comparison of streamlines and trajectories, 1000- $600 \mathrm{hPa}$}

In Sect. 3, streamlines in the co-moving frame were used as a surrogate for parcel trajectories to delineate the critical layer. Although trajectories and streamlines generally differ for unsteady flow, we demonstrate in this section that the translating streamlines so constructed serve as a reasonable first approximation within a few days of genesis. Only the horizontal (isobaric) component of motion is considered here. For a circular vortex moving at a constant speed $C_{p}$, the 

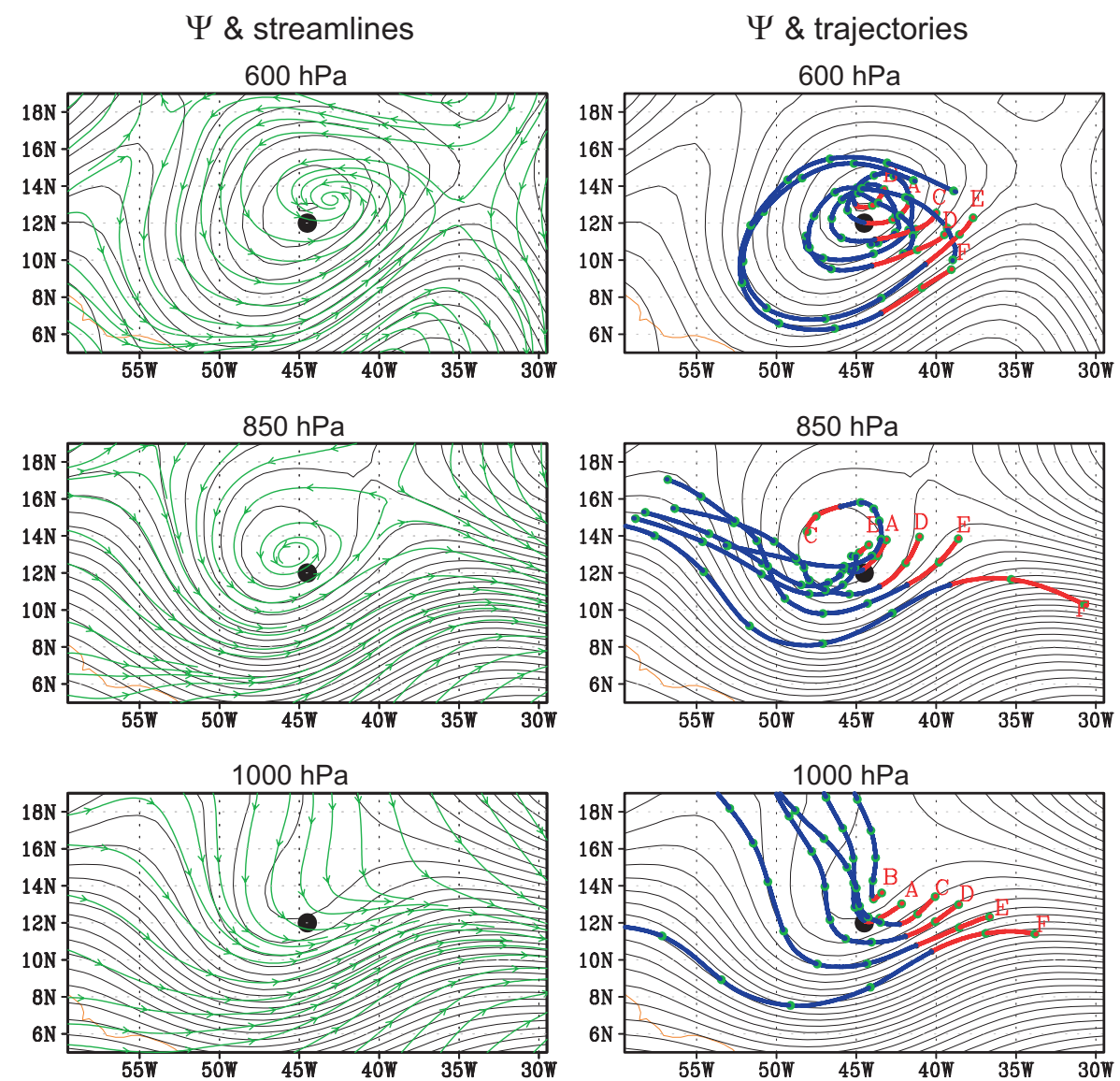

Fig. 18. Streamlines and trajectories of horizontal (rotational + divergent) flow at (a, b) $600 \mathrm{hPa},(\mathbf{c}, \mathbf{d}) 850 \mathrm{hPa}$, and (e, f) $1000 \mathrm{hPa}$ as seen in ERA-40 data, for the average of six consecutive leading up to the best-track genesis time of Hurricane Debby (2000). As in Fig. 5, the streamlines (shown in panels a, c, e) are calculated and displayed in a frame of reference moving westward with the parent wave. Translation speeds estimated from the Hovmöller method at the three levels are $-9.1 \pm 0.3 \mathrm{~ms}^{-1}(600 \mathrm{hPa}),-10.0 \pm 0.6 \mathrm{~ms}{ }^{-1}(850 \mathrm{hPa})$ and $-10.1 \pm 0.6 \mathrm{~ms}^{-1}(1000 \mathrm{hPa})$. Likewise the stream function of non-divergent flow (thin solid) and trajectories (shown in panels b, $\left.\mathrm{d}, \mathrm{f}\right)$ are calculated and is displayed in the co-moving frame. The contour interval for stream function is $0.5 \times 10^{6} \mathrm{~m}^{2} \mathrm{~s}^{-1}$. The genesis location is indicated by the black dot in each panel. Note that owing to the translation of sequential frames used to construct the time average when $C_{p} \neq 0$, the longitude labels on the $\mathrm{x}$-axis pertain to the final analysis time only.

relationship between the local curvature of the streamlines $\left(r_{s}\right)$ and the local curvature of the trajectories $\left(r_{t}\right)$ is

$r_{s}=r_{t}\left(1-\frac{C_{p} \cos \gamma}{V}\right)$

(Holton, 2004) where $V$ is the horizontal wind speed in a natural coordinate (not just the zonal component), and $\gamma$ is the angle between the streamlines and the direction of the vortex translation. For a cyclonic vortex moving westward (eastward), the curvature of the trajectories is stronger (weaker) than that of the streamlines south of the center of the vortex and weaker (stronger) north of the center. For a vortex embedded in and advected by a constant mean flow, the trajectories are corkscrew in shape due to rotation and translation of the vortex. In a frame of reference moving at the horizontal speed $C_{r}, X_{L}=\left(x_{L}, y_{L}\right)^{T}=X-X_{c}$ represent the cardinal coordinates moving with the vortex, and
$X_{c}=\left(x_{c}, 0\right)=\left(C_{r}\left(t-t_{0}\right), 0\right)$ is the center of the vortex. Particle trajectories in this frame of reference satisfy the following equations

$$
\left\{\begin{array}{l}
\frac{d x_{L}}{d t}=\frac{d x}{d t}-\frac{d x_{c}}{d t}=u-C_{r} \\
\frac{d u_{L}}{d t}=\frac{d y}{d t}=v
\end{array}\right.
$$

As illustrated in Fig. 3, if $C_{r}=C_{p}$ streamlines in the comoving frame are stationary and coincide exactly with horizontal trajectories. If the westward phase speed is underestimated (overestimated) the curvature of trajectories is stronger (weaker) than that of streamlines south of the center of the vortex and weaker (stronger) north of the center. Here we will use the formation of Hurricane Debby to examine the three-dimensional structure and evolution of the critical layer. 
Figure 18, left column, shows streamlines at 600,850 and $1000 \mathrm{hPa}$ in a co-moving frame averaged over the 36 -h period leading up the genesis time of Hurricane Debby. Also shown is the stream function obtained from the horizontal velocity components by a Helmholtz decomposition. Streamlines and stream function contours are nearly parallel at $850 \mathrm{hPa}$, indicating a non-divergent flow, except near South America where air flows into the gyre from the west. The $600 \mathrm{hPa}$ flow is almost non-divergent as well. The Helmholtz decomposition is linear, so that transient divergent flows are eliminated by time averaging, or if they are small to begin with (as true at these two levels for the individual analyses used in the time average) the divergence remains small when averaged in time. The exact location of flow separatrix and its hyperbolic point(s) is evidently sensitive to the small divergent component, because the horizontal wind speed vanishes at this point (by definition) whereas for stream function alone, the critical point is located where the nondivergent component of horizontal wind speed is zero. Note that, despite this error, the separatrix inferred from stream function is within $100-300 \mathrm{~km}$ of the separatrix inferred from streamlines. Unlike the first two levels, the $1000 \mathrm{hPa}$ level displays significant convergence into the gyre from the north and west, and into a zonally elongated ITCZ in the eastern half of the figure, from north and south. This feature is described in Sect. 4.3. The flow entering the gyre from the north makes little effort, as it were, to avoid the center of the gyre by turning to the west, as at the other two levels. Although the genesis location (black dot) is $\sim 100 \mathrm{~km}$ south of the gyre center at all three levels, the exact latitude of center is sensitive to our choice of $C_{p}$, moving $\sim 80 \mathrm{~km}$ southward for each $1 \mathrm{~ms}^{-1}$ increase of $C_{p}$ towards zero (Generally this depends on vorticity or circulation. A weaker circulation implies a stronger sensitivity to $C_{p}$ ). Values of $C_{p}=-8$ to $-9 \mathrm{~ms}^{-1}$ place the $1000 \mathrm{hPa}$ closed gyre precisely over the genesis location. These values are outside the range of uncertainty based on the Hovmöller method, but are optimum for steadiness of flow in the translating frame, as described in Sect. 4.2. Steadiness in the translating frame implies that streamlines and trajectories should be similar in this frame. To settle the question of which translation speed is best, streamlines and trajectories can be compared.

Figure 18, right column, shows 2-D parcel trajectories superimposed on the stream function averaged over the same time interval. The trajectories are calculated in the comoving frame, as shown in Eq. (3). The red curves are one-day forward trajectories from day 0 to day +1 , and the blue curves are four-day backward trajectories from day 0 to day -4 . (Trajectories are not shown outside of the domain.) The gyre at $600 \mathrm{hPa}$, indicated by closed stream function contours, is quite expansive. Six particles are picked, five of them (A-E) inside the gyre at the genesis time, the other one (F) just outside of the gyre. The particles moved counterclockwise around the gyre center, and their trajectories are nearly parallel to the stream function contours from day -2 to day +1 . Since the flow is non-stationary, the trajectories are different from the stream function contours or streamlines from day -4 to day -2 , but it nevertheless confirms our hypothesis that particles inside the critical-layer cat's eye tend to stay inside. Compared to $600 \mathrm{hPa}$, the gyre at $850 \mathrm{hPa}$ is less expansive. The evolution of the streamlines (not shown) showed that the wave at $850 \mathrm{hPa}$ propagated on the northern flank of the AEJ over West Africa. It turned southward and merged with the wave on the southern flank (maximum at $600 \mathrm{hPa}$ ) off the west coast, and meanwhile the closed circulation extended to the surface. A tropical depression formed within the wave about two days later. Six particles are picked at $850 \mathrm{hPa}$ : particles $\mathrm{A}-\mathrm{C}$ are inside the gyre and D-F outside the gyre. Particles D-F moved from west to east in the co-moving frame, and stayed outside of the gyre. Particles A-C also came from the west. They took a small loop, and entered the gyre around day -2 . This is consistent with the formation of the gyre at $850 \mathrm{hPa}$ around day -2 , as shown by the evolution of the streamlines. The trajectories of all the six particles are nearly parallel to the stream function contours from day -2 to day +1 . At $1000 \mathrm{hPa}$, the gyre is much more confined. Particle A is picked near the genesis location, $\mathrm{B}$ close to the gyre center, and $\mathrm{C}-\mathrm{F}$ outside of the gyre. Particles A-E came from the north, and particle F, which is far from the gyre center, moved from the west, which suggests that the closed circulation near the surface is directly related to the northern wave.

To test the robustness of this result, we increased the sample size by examining the trajectories of 40 particles inside or just outside of the gyre at each of the three vertical levels (not shown). Similar to what is shown in Fig. 18, the particles inside of the gyre tend to stay inside from day -2 to day +1 , and all the trajectories are nearly parallel to the stream function contours during this time interval. This suggests that the stream function in the frame of reference moving with the wave serve as a reasonable first approximation within about 2 days of genesis. From the trajectories, we can also estimate the recirculation time of a parcel inside the CL or the eddy turnaround time, which is about 2-3 days and close to the tropical storm development time scale. This is also the length of time that streamlines, stream function and trajectories are roughly equivalent near the gyre center. Table 1 includes two estimates of gyre recirculation time, one derived from the maximum vorticity in the gyre, the other from the average Okubo-Weiss parameter in the cyclonic vorticity region only. The maximum vorticity estimate (typically $2-5$ days) is shorter than the $\mathrm{O}-\mathrm{W}$ estimate (typically $3-8$ days) because the latter pertains to a broader region of the pouch, and subtracts the contributions from straining and shearing deformation. Actual recirculation times probably fall between these two extremes. Note, however, that horizontal convergence in the boundary layer shortens the recirculation time as the effective radius of trajectory curvature contracts while parcels begin their ascent near the proto-storm center. 


\subsection{Vertical structure and evolution of the mother pouch}

The need to translate the coordinate system zonally for an optimum view of Lagrangian flow kinematics has been stressed throughout the paper. Towards this end, a method for determining the optimum translation speed was devised using bandpass meridional velocity anomalies in time-longitude Hovmöller diagrams just prior to genesis. The purpose of the translation is to render flow streamlines approximately equivalent to trajectories, as demonstrated in the preceding subsection. If one were to instead measure explicitly the temporal variance of the flow in a coordinate system translating zonally at an arbitrary speed, it would be found that temporal variance in the vicinity of the developing storm is indeed minimized at a phase speed close to that selected by the Hovmöller method. The reason is that when one chooses an incorrect phase speed for translation, the wave's phase propagation contributes significantly to the temporal variance in the incorrectly translating frame. When the proper translation speed is selected, this temporal variance is mostly eliminated. The proper speed pertains to that of the "co-moving" frame as defined herein. We have applied the alternative method to stream function (as opposed to streamline) which represents a field, that is, function of space and time (unlike particles whose location is simply a function of time). Because the flow is dominated by the rotational ${ }^{26}$ component, stream function in the co-moving frame nicely illustrates the structure and evolution of the parent wave's "pouch". Representative examples of stream function for the mother pouch of Debby were shown in Fig. 18. The definition of Lagrangian boundary in steady flow can be made precise by locating the saddle point nearest (in psi) to the gyre center and equating the gyre boundary with the value of stream function intersecting that point.

In regard to the vertical structure of Debby's pouch, two salient features emerge with other interesting details. (i) A closed gyre is found at all levels from $1000-500 \mathrm{hPa}$ for at least $30 \mathrm{~h}$ prior to genesis, for westward translation speeds in the range -8 to $-10 \mathrm{~ms}^{-1}$. The signature of this disturbance decreases abruptly in amplitude above $500 \mathrm{hPa}$. (ii) Above the mid-troposphere is a second closed anticyclonic gyre having somewhat larger spatial extent and located slightly to the east of center, but a significantly slower range of possible westward translation speeds in the range -3 to $-7 \mathrm{~ms}^{-1}$. Close inspection of gyre properties, such as the steadiness of pattern, stationary center location and minimum temporal variance near the center (all evaluated in the translating frame) establishes a pair of optimum westward translation

\footnotetext{
${ }^{26}$ The rotational component is larger in most places except, e.g., the upper branch of the local Hadley circulation, where the divergent component is comparable in a relatively shallow layer near $200 \mathrm{hPa}$ (Dunkerton, 1995) and in the lower branch of this circulation, or boundary layer.
}

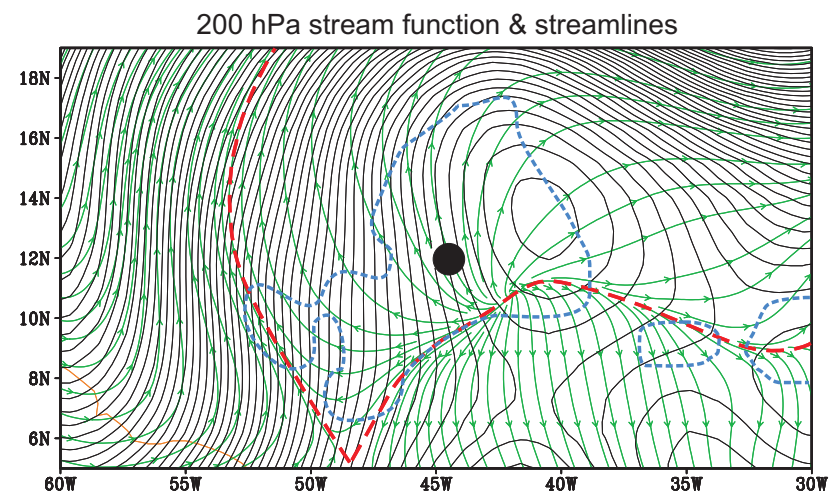

Fig. 19. Streamlines of horizontal flow and stream function of nondivergent flow at $200 \mathrm{hPa}$ calculated and displayed in a frame of reference moving $5 \mathrm{~ms}^{-1}$ westward, at the genesis time of Hurricane Debby. Divergence contributing to anticyclonic outflow can be seen by comparing streamlines with stream function. The genesis location is indicated by the black dot; TRMM precipitation is outlined in blue.

speeds: $-9 \mathrm{~ms}^{-1}$ in the lower troposphere and $-5 \mathrm{~ms}^{-1}$ in the upper troposphere. Streamlines and stream function at $200 \mathrm{hPa}$ in the $-5 \mathrm{~ms}^{-1}$ gauge at genesis time are shown in Fig. 19. The two signals $\left(-5\right.$ and $\left.-9 \mathrm{~ms}^{-1}\right)$ are found to have a common origin in the convective flare-up at $36-38^{\circ} \mathrm{W}$ at $30 \mathrm{~h}$ before genesis (Fig. 9a) explaining why the upper tropospheric signal is located to the east of the storm center at genesis time. The LT wave continues its westward propagation near $-9 \mathrm{~ms}^{-1}$, reaching the genesis location $30 \mathrm{~h}$ later, while the UT anticyclone drifts westward about half as fast, reaching $40-42^{\circ} \mathrm{W}$ as shown in Fig. 19. Stream function is a reasonably good proxy for streamlines over the left and upper halves of the figure, but misses completely the strongly divergent outflow above the proto-storm center as well as the local Hadley circulation outflow (along the ITCZ to the east) directed back to the Equator.

Mean vertical shear over the troposphere is relatively light in this case. The flow speed in the upper troposphere near $300 \mathrm{hPa}$ is about $-4 \mathrm{~ms}^{-1}$, so that when the coordinate system translates westward near this speed, most of the jet structure disappears, leaving an anticyclonic gyre slightly to the east of the genesis location, with other meridional meanderings elsewhere. As a result, weak southerly flow $\left(3 \mathrm{~ms}^{-1}\right)$ occurs over the depression at genesis time, in the co-moving frame as shown in Fig. 19. (The meridional component, of course, does not depend on the choice of translation speed, or gauge, when the translation is zonal. It is the vanishing of zonal component in the co-moving frame that makes the flow appear southerly in this frame, rather than westsouthwesterly as in the resting frame.) The UT gyre may be significant for development by providing favorably light winds above the proto-storm. The developing storm itself may have had a role to play in carving out the UT anticyclone 
as a result of a convective event at $36-38^{\circ} \mathrm{W}$ (next subsection). Averaged over the $36 \mathrm{~h}$ (6 consecutive analyses) prior to genesis, the structure of relative wind speed (modulus of vector $\mathbf{U}$ minus $\left.C_{p} \hat{\mathbf{i}}\right)$ for two values of $C_{p}$ ( -9 and $-5 \mathrm{~ms}^{-1}$ for LT and UT, respectively) is shown in Fig. 20a, b. The temporal standard deviation of wind speed over the same time interval is also shown (c, d). Contours indicate a zonal-temporal average over a $30^{\circ}$ longitude interval eventually centered at the genesis point and translating zonally prior to this time for $30 \mathrm{~h}$ at speed $C_{p}$. These wave-centric views illustrate in approximate fashion the structure of the mother pouch in a latitude-height cross section. Superposed as shaded pixels are wind speed and its temporal standard deviation within the boundaries of the translating closed gyres in each frame, as defined by their maximum extent in this longitude/time interval. As already noted, the purpose of the translation is to minimize the temporal variation of streamlines in the translating frame, rendering streamlines approximately equivalent to trajectories. Comparison of Fig. 20c, $\mathrm{d}$ indicates that the temporal variation of wind speed within the gyre over $36 \mathrm{~h}$ is considerably smaller in the LT when the translation speed is set at $-9 \mathrm{~ms}^{-1}\left(0.3-0.7 \mathrm{~ms}^{-1}\right)$ than at $-5 \mathrm{~ms}^{-1}\left(0.7-1.6 \mathrm{~ms}^{-1}\right)$ whereas a value of $-5 \mathrm{~ms}^{-1}$ is superior for steadiness of the UT anticyclone (a closed a/c gyre at these altitudes does not appear at all at $\left.-9 \mathrm{~ms}^{-1}\right)$. When the translation speed is set at $-9 \mathrm{~ms}^{-1}$, relative wind speeds in the LT are smaller inside the gyre $\left(1.4-3.4 \mathrm{~ms}^{-1}\right)$ than over all longitudes $\left(4-6 \mathrm{~ms}^{-1}\right)$, whereas at $-5 \mathrm{~ms}^{-1}$, these values $\left(4-6 \mathrm{~ms}^{-1}\right)$ are comparable. The moral of the story: the slower translation speed is inappropriate in the lower troposphere, and its effect in reducing the relative flow or variation thereof inside the LT gyre is inconsequential. It is easy to understand why the temporal variation is minimized at $-9 \mathrm{~ms}^{-1}$, because the wave's phase propagation has been accounted for. This translation speed closely approximates that of the LT wave, while the slower speed does not. The reason why the gyre's relative flow is reduced is more subtle. Like the hyperbolic point on the separatrix, the gyre center is a stagnation point where the relative flow is weak, albeit rotating cyclonically in such a way as to make a complete revolution about gyre center in a few days or less. Both choices of translation speed yield a closed translating gyre, implying a stagnation point either way, so why is the faster translation speed ostensibly superior in the metrics of Fig. 20? The "secret of success" at $-9 \mathrm{~ms}^{-1}$ is that the gyre keeps perfect step with the wave and is not too large in horizontal extent. In the $-5 \mathrm{~ms}^{-1}$ gauge the gyre is much larger and includes a peripheral region of stronger relative flow. This peripheral flow is so strong, in fact, that the gyre-average relative flow (shaded pixels in Fig. 20c) is stronger than that of all longitudes (contours)! We emphasize, however, that small relative flow per se is not necessarily significant. It will be noted (Fig. 20b) that a small relative flow $\left(1-2 \mathrm{~ms}^{-1}\right.$ ) is also observed at $400 \mathrm{hPa}$ near the genesis latitude when the translation speed is set at $-5 \mathrm{~ms}^{-1}$. There is no closed gyre at this level; rather, the selected translation speed is similar to the zonal flow speed here. Translation eliminates the zonal component of relative flow at $400 \mathrm{hPa}$, leaving weak meridional meanderings at this level which have little, if any, importance dynamically - other than to ensure weak relative flow above the proto-storm.

In regard to temporal evolution, a closed LT gyre in the co-moving frame can be found all the way back to $54 \mathrm{~h}$ before genesis time. The traveling gyre emerges from a quasistationary gyre near the west coast of Africa, presumably a monsoonal feature. The exact time of gyre closure is a function of altitude and translation speed $C_{p}$. A closed surface circulation exists at least $60 \mathrm{~h}$ prior to genesis in the $-5 \mathrm{~ms}^{-1}$ gauge, but as already noted, this speed is inappropriate for the LT wave ${ }^{27}$. In the properly co-moving frame $\left(-9 \mathrm{~ms}^{-1}\right)$ the surface circulation closes at 18:00 Z on 17 August, $48 \mathrm{~h}$ prior to best-track genesis. There is an implied delay (albeit short) between the formation of a closed circulation in the lower free troposphere relative to the surface. It remains to be determined whether downward burrowing of a closed gyre to the surface from above is a universal behavior in all 55 cases; the largest and/or earliest translating gyres can be found at any of the analyzed LT levels $(1000-600 \mathrm{hPa})$ depending on the storm. A closed surface circulation, nonetheless, is present in most cases together with a modest vertical shear extending to the UT and other environmental stimulants as identified in Figs. 16-17. These observations confirm $\mathrm{H} 1$, establishing that the locus of gyre formation and organization of deep convection is in the lower half of the troposphere, while leaving some important details (e.g., communication between the lower free troposphere and boundary layer) for future study. On balance, conditions throughout the depth of the troposphere in this event seem very favorable for tropical cyclogenesis via the tropical wave pathway. But as already noted, Debby was not unique in this respect. Similar conditions are found in most of the cases selected for the "quasi-monochromatic easterly wave" category.

\subsection{Signatures of wave and diabatic vortex prior to genesis}

A distinct signature of the parent wave and diabatic protovortex just prior to genesis can be seen in Debby and in other cases examined, that is, a set of defining characteristics that help to discriminate these two entities. Recognition of how these characteristics evolve in the day or two immediately preceding genesis may prove useful for diagnosis and prediction of incipient storm development. The vertical structure of

\footnotetext{
${ }^{27}$ Monsoon circulations (tied to stationary land surfaces) fluctuate slowly in time, implying a non-zero bandwidth in frequency. Closed monsoon gyres are therefore expected in a modest spread of translation speeds, as observed in this case. Monsoons do not give birth to tropical depressions, but tropical waves and monsoon depressions do. For genesis it is not the translation speed of monsoon circulations that is important, but that of waves.
} 

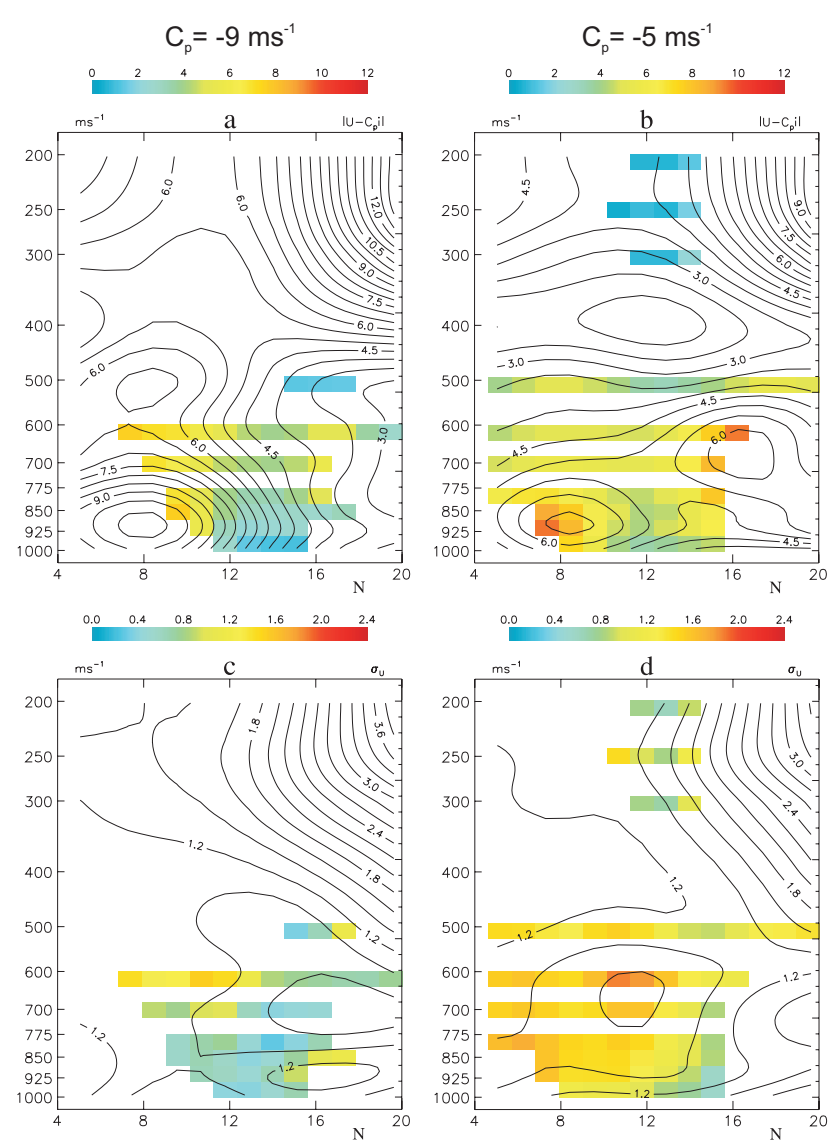

Fig. 20. Horizontal wind speed in two co-moving frames averaged over the six consecutive analyses leading up to the genesis time of Hurricane Debby. The range of longitudes $x$ used to construct the average is given by the speed of translation beginning at the genesis longitude $\left(x_{0}=44.5^{\circ} \mathrm{W}\right)$ and going eastward (backward) in longitude (time) from the genesis point according to the formula $x(t)=x_{0}-C_{p} \cdot\left(t-t_{0}\right)$ where $t_{0}$ is the best-track genesis time. The horizontal wind speed in each co-moving frame is given by $\left|\mathbf{U}-C_{p} \hat{\mathbf{1}}\right|$ where $\mathbf{U}$ is the horizontal vector wind, $C_{p}$ is translation speed and $\hat{\mathbf{i}}$ is the unit vector in the $x$-direction. The quantity contoured represents a zonal average within $\pm 15^{\circ}$ longitude of the center longitude as given above, including regions inside and outside the gyre. (a, c) $C_{p}=-9 \mathrm{~ms}^{-1} ;(\mathbf{b}, \mathbf{d}) C_{p}=-5 \mathrm{~ms}^{-1}$ (negative values are westward). These values correspond approximately to the zonal component of disturbance propagation in the lower and upper troposphere, respectively. The maximum extent of closed stream function gyre in each frame of reference is also shown by superposing (as shaded pixels) the gyre-average values of $\left|\mathbf{U}-C_{p} \hat{\mathbf{1}}\right|$ where they exist. Here, "gyre average" refers to a zonal average over longitudes within a closed gyre as delimited by a bounding contour of stream function $\psi$ intersecting the nearest (in $\psi$ ) separatrix. The lower panels show the temporal standard deviation of horizontal wind speed in each co-moving frame. The quantity contoured once again represents a zonal average over the entire domain, while shaded pixels indicate gyre-average values. For modulus and standard deviation, their zonal average was obtained by first summing the squares and then taking the square root of the sum. wave and vortex seem especially important in this regard. As noted in the preceding subsection, the parent wave of Debby translates westward at about $8-10 \mathrm{~ms}^{-1}$, with little vertical phase tilt up to the mid-troposphere and abrupt decrease of amplitude above. Meanwhile, an extensive anticyclonic gyre develops in the upper troposphere and propagates west, but with slower phase speed than the wave, about 5-6 $\mathrm{ms}^{-1}$ westward. It is thought that the UT anticyclone may have originated from diabatic forcing by deep moist convection at $36-38^{\circ} \mathrm{W}$ about $30 \mathrm{~h}$ prior to genesis time. Figure 9 indicates that persistent convection was seen by TRMM during this sequence, aligned well with the translating gyre, especially at the beginning. Remarkably, the ERA-40 stream function and velocity potential, when examined together, seem to confirm these two signatures - noting the important caveat that the analyzed divergence is driven in part by the model's cumulus parameterization and is therefore uncertain. Velocity potential in the upper troposphere indicates a strong divergence anomaly in the upper troposphere propagating westward during the $30-\mathrm{h}$ sequence from $36-38^{\circ} \mathrm{W}$ to the exact location of genesis at genesis time, accompanied by a convergence anomaly in the lower troposphere propagating westward in parallel, 1 or $2^{\circ}$ south of this track as shown in Fig. 21a-d . The vertical alignment of these anomalies of opposite sign is similar to the "first baroclinic mode" structure of tropical depression disturbances in the western Pacific (Dunkerton, 1993) but very different from the vertically trapped, equivalent barotropic structure typical of Atlantic easterly waves that are not diabatically activated. We conjecture that a vertical dipole of velocity potential (accompanied by a UT anticyclone and vertical quadrupole of meridional velocity anomaly) indicates activation by one or more diabatic vortices and their associated deep moist convection within the mother pouch of the wave. In Debby and in other cases, excitation of a divergent UT anticyclone above the LT disturbance provides a spectacular early warning signal in the dynamical fields that seems to corroborate well with convective precipitation retrieved from TRMM measurements.

Forecasters consider in advance various signatures of TD genesis; for some of them, the upper tropospheric flow plays a role, as in vertical shear, upper-level diffluence or divergence, and of course, deep convective cloud-top temperature. It is often impossible to distinguish cause and effect, since one could just as well interpret their presence as indicating that a tropical depression already exists, but has not been officially recognized. The main hurdle in identifying a TDstage disturbance is the inability to see a closed rotational flow in the lower troposphere in visible or infrared imagery when obscured by clouds aloft. In this respect it is worthwhile to recognize the often crucial role played by the upper tropospheric flow in identifying conditions which either are favorable to development or indicate that development is already in progress. Owing to improvement in the assimilation of cloud and water-vapor winds in operational analyses over the last two decades (e.g., Velden, 1997, 2005) the free 


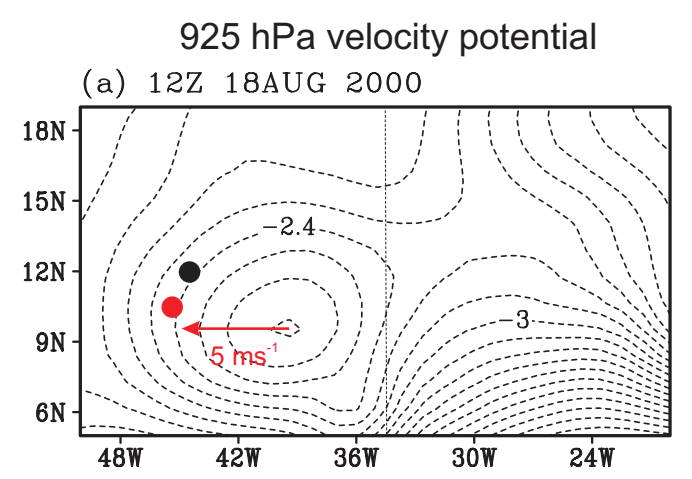

(c) $18 \mathrm{Z}$ 19AUG 2000

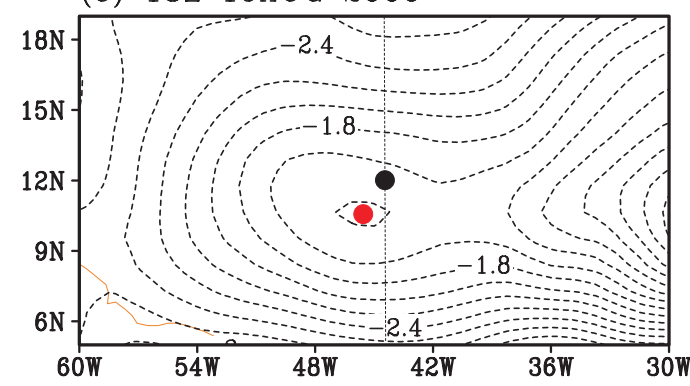

$200 \mathrm{hPa}$ velocity potential

(b) $12 \mathrm{Z}$ 18AUG 2000

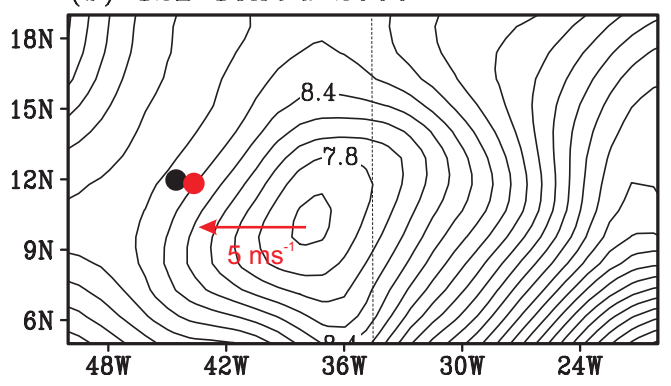

(d) $18 \mathrm{Z}$ 19AUG 2000

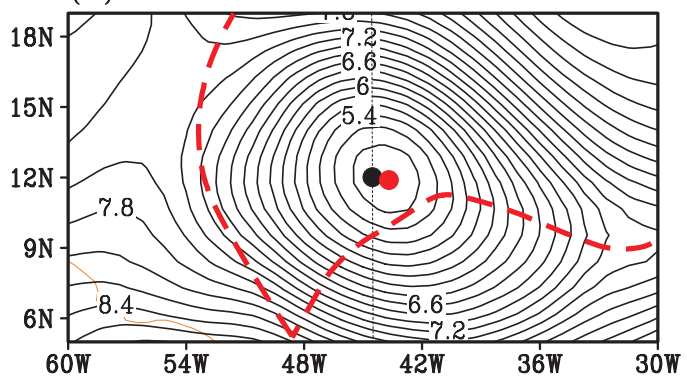

Fig. 21. Velocity potential of divergent flow, as seen in ERA-40 data, at (a, c) $925 \mathrm{hPa}$ and (b, d) $200 \mathrm{hPa}$ leading up to the genesis time of Hurricane Debby, at (a, b) $30 \mathrm{~h}$ before genesis and (c, d) genesis.

troposphere flow is well-represented on the synoptic scale (a compliment that excludes the tropical tropopause layer or TTL, and the lower stratosphere, due to insufficient cloud coverage or water vapor content, and lower troposphere in regions where thick clouds obscure). To the extent that both lower and upper troposphere horizontal winds are captured faithfully by the analyses on the synoptic scale, it is possible to identify TD-like conditions in the parent wave from the vertical structure of wind anomalies: specifically, a "first baroclinic mode" structure or stacked arrangement of LT cyclone and UT anticyclone.

Such features - as one might expect us to say - are best revealed in a frame of reference translating with the parent wave. But in the case of Debby and more generally, there is no need for the optimum translation speeds to be identical throughout the depth of the troposphere. One reason (noted in Sect. 3 and quantified in Table 1) is that the phase speed of the parent wave may vary with height, from lower to mid-troposphere. Another (noted here) is that the protovortex may translate slowly with respect to its parent wave while its deep convective signature extends to the upper troposphere $^{28}$. The definition of "properly co-moving frame" therefore depends precisely on what the "co" refers to. In-

\footnotetext{
${ }^{28}$ Effects of the diabatic proto-vortex on the upper troposphere may be separated into a near-field response with anticyclonic outflow aloft (relevant to TC genesis within) and a far-field response communicated by secondary Rossby waves (relevant to adjacent troughs and TC genesis therein).
}

deed, it is likely that a trapped LT disturbance propagates at a slightly different speed than a diabatically activated LT-UT dipole. There are multiple reasons, the simplest being that gross moist stability is reduced by the latent heating associated with deep moist convective precipitation, causing wave phase speed of moister waves to be slower than that of drier waves. In the language of tidal theory it could be said that the equivalent depth of the proto-vortex and its induced flow is smaller than that of the original parent wave, which sees a larger area and more dilute distribution of precipitation than the proto-vortex itself and its upper tropospheric signature. We therefore expect a diabatic Rossby wave and diabatic Rossby vortex to propagate at slightly different speeds, the speed of the wave depending, among other things, on the degree of convective heating seen by the wave, via the gross moist stability. Also possible is that the two entities respond differently to vertical shear. An isolated vortex is expected to propagate at the speed of the local mean flow (excluding the effects of unbalanced motions, if any) which, as noted in Sect. 2, matches the phase speed of the wave at the critical latitude. There are kinematic reasons for the parent wave and proto-vortex to remain together, at least within some maximum distance as determined by the dimensions of the translating gyre. But they do not necessarily walk in lock step. The marsupial paradigm evidently allows some "slop" in the exact position of the vortex relative to the wave trough, i.e., slightly different propagation speeds which (as in Debby and other cases) are measurably different. Key to the success 
of the marsupial mechanism is that the respective horizontal velocities not differ too much either in speed or azimuthal direction.

Broadly speaking, for diagnostic purposes and short-term prognosis, we conclude that (i) stream function in the lower to mid-troposphere suitably illustrates the incident wave structure, along with velocity potential in the lower free troposphere above the boundary layer, while (ii) velocity potential in the boundary layer and upper troposphere, along with stream function in the upper troposphere, depicts the diabatic vortex and/or modification of wave structure once diabatic activation has occurred.

\section{Conclusions, new visions and directions}

The genesis of tropical cyclones was described by Emanuel (2005) as one of the remaining "great mysteries of the tropical atmosphere." The persistence of the mystery over many decades underscores the difficulty of the problem: like the conquest of Mount Everest, a formidable "ascent" from observational, numerical and theoretical directions. Some of the processes involved in genesis occur at horizontal scales which are very small and unresolved by analyses such as ERA-40 on a $\sim 1$ degree grid. Numerical models need much finer resolution than this in order to capture the mesoscale construction of the incipient PV monolith in a realistic manner (e.g., M06). But the mystery also exists owing to imperfect theoretical understanding and visualization of fluid dynamics and thermodynamics that comes from working in an Eulerian or Earth-relative framework. We have produced and advocated in this paper a Lagrangian or wave-centric view for the study of tropical cyclogenesis and have chosen the simplest of all scenarios to present the fundamental concepts of genesis in a tropical wave critical layer. These events were associated with quasi-monochromatic easterly wave disturbances which account for the majority of tropical cyclones in the Atlantic and eastern Pacific sectors (if we allow in some cases for hydrodynamic instability to account for the waves themselves). To the extent that the phase speed of waves is reasonably constant over their life cycle, the coordinate system may be translated westward to match the phase speed of the incident wave. By examining streamlines in the co-moving frame a wave-centric picture of the flow is obtained in which trajectories and streamlines are nearly equivalent over a time span of a few days. Because the divergent component of horizontal flow is small relative to the rotating component, with certain exceptions, streamlines in the translating frame are closely approximated by a stream function with the appropriate "gauge" (in this case, a scalar harmonic function) added corresponding to the speed of translation. This device enables us to identify approximate Lagrangian boundaries in the flow in lieu of performing trajectory calculations. To confirm inferences from streamlines in the co-moving frame we calculated back-trajectories from within the closed gyre (Sect. 4.1).

The marsupial paradigm provides a convenient and self-consistent framework for the coupled dynami$\mathrm{cal} /$ thermodynamical processes that are essential to tropical cyclogenesis. Our analysis has highlighted the role of tropical wave critical layers as preferred loci for the formation and aggregation of diabatic vortices which represent moist coherent structures on the mesoscale. The critical layer is where the mean flow (in the direction of wave propagation) and wave phase speed are similar, implying that cutoff vortices will be able to travel with the wave (in this case, westward) for a time. The coherent relationship that exists between the wave and vortex serves to preserve both. It preserves the vortex because the Lagrangian boundaries formed by the wave at its critical layer remain in the same phase of the wave. These boundaries appear stationary or slowly varying in the co-moving frame. Also, diabatic heating associated with the spawned vortices acts coherently to maintain the wave to the extent that these vortices are able or inclined to keep step with the wave, as implied by the preceding argument. This phase locking, however, does not persist indefinitely, and when a vortex becomes sufficiently strong $^{29}$ it may acquire its own propagation characteristics which usually involves some degree of meridional propagation that the wave did not have originally as well as a slower horizontal speed. The vortex is then no longer propagating with its parent wave. This sequence of events we have described as the marsupial paradigm because the wave is providing a pouch or sweet spot for cyclonic vortex development, up-scale aggregation, convective moistening and diabatic amplification. This process has a finite gestation period. Once the vortex leaves, the wave is often observed to weaken or die when viewed in NHC/TPC surface analyses. Multi-dimensional visualization of ERA-40 data suggests also a subsequent radiation of waves, either from the genesis region itself or from the new translating vortex, that introduces new variance in the upper troposphere.

Our third hypothesis (H3) is consistent with these observations; quantitative proof is outside the scope of this paper. Phase-locking of wave and proto-vortex for \pm 3 days about the best-track genesis time is more prominent in stronger waves than in weaker waves, the latter seeming to exert less influence on the direction of vortex propagation. As for the effect of vortex on wave, there are numerous instances in which the speed of zonal phase propagation is reduced after genesis, although this behavior is not universal. Model results (to be reported separately) confirm that a diabatically activated easterly wave is slowed, by about $20 \%$, relative to its initial speed prior to vortex development. Observations from ERA-40 indicate a prominent signature of

\footnotetext{
${ }^{29}$ Our modeling study establishes that vortex merger and convective amplification play equally important roles in the selection of a dominant vortex in the pouch (Montgomery et al., 2009).
} 
diabatic activation in the upper troposphere above the developing storm, as seen by a quadrupole structure of meridional velocity anomaly in the longitude-height plane. Similar structure comprised the leading mode of meridional velocity variance in the western Pacific during boreal summer (Dunkerton, 1993). Its occasional presence in Atlantic and eastern Pacific sectors, uniquely associated with TC genesis in these regions, may prove important in diagnosis and short-term prediction. Forecasters show keen interest in anticyclonic outflow in the UT when it exists prior to intensification. Divergence and anticyclonic rotation above the proto-storm adds a piece of information to the marsupial paradigm and supports the notion that the mother gyre and child vortex have different personalities, i.e., contrasting vertical structures and slightly different horizontal propagation speed and/or direction.

These ideas provide a road map for exploration of synoptic-mesoscale linkages essential to tropical cyclogenesis, an exciting area of research in the years ahead. For tropical cyclogenesis in tropical waves, our analysis begins to close the gap between observationally resolvable scales (synoptic, meso- $\alpha$ ) and cloud scales (meso- $\gamma$ ) that require numerical models. To recap: the formation, aggregation and amplification of preferentially cyclonic anomalies by convective heating in the Kelvin cat's eye of a critical layer comprise the principal pathway for tropical cyclogenesis in tropical waves (H1). A key concept derived from theories of the nonlinear critical layer is that the cat's eye exists prior to organization and does not depend, structurally or otherwise, on the details of organization until TC intensification begins. The formation of closed streamlines implies that the air within the materially closed space is protected, to some degree, from lateral entrainment of dry air $(\mathrm{H} 2)$. Therefore the closure serves not only to concentrate vorticity by the aforementioned mechanism but may also account for the accumulation of moist entropy and saturation of the tropospheric column by persistent deep moist convection in the pouch - the same convection that facilitates growth of cyclonic anomalies. This containment has a very important effect in allowing a convective type of heating profile to dominate the closed region with a relatively lesser contribution from stratiform precipitation that is otherwise common in meso-scale convective systems (Tory and Montgomery, 2006). The maximum heating in the immediate region of interest occurs at a lower altitude than normal for organized tropical convection, favoring surface convergence and rapid spin-up of one or more lowlevel vortices. We conjecture that this process, representing a "non-advective" transport of PV (Haynes and McIntyre, 1987) by hot towers in an environment of large saturation fraction (exceeding $\sim 80 \%$ ) provides a direct "bottom-up" pathway to tropical cyclogenesis more or less independent of the mid-level transport of PV by typical mesoscale convective systems in the tropics.

Persistent deep convection in the gyre and its signature in the upper troposphere observed prior to official TD declara- tion suggests that anomalous diabatic processes are already operating in this region: anomalous with respect to temporal duration, vertical profile of heating and the resulting profiles of vorticity and divergence. How these anomalies translate into TC formation remains something of a mystery because the mesoscale processes most relevant to storm development are uncertain, while available observations lack sufficient resolution. For this reason, we cannot establish that gyre formation in the co-moving frame is completely sufficient for tropical depression formation. Nonetheless, a critical role for a closed surface gyre is supported by these observations. We have associated (i) gyre depth (equivalently, vertical alignment) and (ii) anomalous dynamical and thermodynamical properties within the gyre, with developing systems, and have used contemporary meteorological analyses and satellite data for this purpose, notwithstanding their limitations. Further insight into mesoscale processes that link gyre formation to ensuing developments will help to establish how far the forward enstrophy cascade penetrates the mesoscale before cloud-system processes trigger the inverse cascade and ultimately dominate the intensification phase via upscale aggregation. Numerical models will be brought to bear on this aspect of the problem. With a Lagrangian framework for flow kinematics (relevant to the forward cascade) and a cloud-system resolving model (needed for the inverse cascade) a direct assault on mesoscale processes leading to tropical cyclogenesis at meso- $\beta$ is now feasible. We have brought the necessary materials to base camp, so to speak, and the summit of the mountain is within reach.

The multi-scale problem just described would be complicated enough if confined to a horizontal plane, but nature provides a vertical dimension, or degree of freedom, absent from the 2-D model. We have attempted to draw some preliminary conclusions from the vertical structure of waves, proto-vortex and mean flow (Sect. 4) but most of the work on this aspect of the problem remains to be done. Identification of a gyre boundary in the co-moving frame enables a quantitative estimate of vertical shear seen by the developing storm, in addition to its intrinsic properties (vorticity, moisture, deep convection and recirculation time; Table 1, Figs. 16-17). It seems that genesis is favored in regions of weak vertical shear when measured over the depth of the troposphere. The shear need not be identically zero, nor as small as the "shear of the climatology", but the typical 850$200 \mathrm{hPa}$ shear at the genesis point observed in our survey of events is modest $\left(<12 \mathrm{~ms}^{-1}\right)$ in agreement with previous studies. Zero shear throughout the gyre would imply that the flow kinematics at any two levels are the same. Of course this is never exactly so. Horizontal transports of cyclonic vorticity and moisture are therefore somewhat different in all cases, but they are seldom radically different. Nor would it be correct to regard TC genesis in these basins as resulting from little more than "stratified turbulence" in which horizontal flows at different levels overlap randomly. Incident waves are usually observed at both levels $(600 \& 850 \mathrm{hPa})$ 
with similar amplitude and phase. In regard to the vertical structure of horizontal transport (of vorticity and moisture) the marsupial paradigm, with its approximately equivalent barotropic structure of lower-tropospheric waves, provides a much more realistic picture of tropical cyclogenesis than the stratified turbulence paradigm. The latter may describe better the relation of upper and lower troposphere, the upper levels often influenced by intrusion of extratropical anomalies uncorrelated with the lower tropical troposphere.

Vertical alignment of the marsupial pouch as a favorable condition for TC genesis helps to mitigate the deleterious effect of vertical shear on the nascent storm:

"A major inhibitor of genesis is the presence of vertical wind shear, defined as the change in direction and/or speed of the wind with altitude... If there is no wind shear, a nascent tropical system just moves along with the wind (which, in the absence of shear, has the same speed and direction at all altitudes). No air flows through the storm. But if wind shear is present, the storm tries to move along at an average wind speed, and at some altitudes wind must blow through the storm. This storm-relative flow can import dry air from outside the cloud cluster, destroying the humid column of air that is needed for genesis. For this reason, wind shear is inimical to genesis" (from Divine Wind: The History and Science of Hurricanes by Kerry Emanuel, 2005, p. 97).

Although additional reasons were noted in Sect. 2, concerning effects of vertical shear on convective organization, two aspects of the marsupial paradigm were anticipated in Emanuel's reasoning: the need for weak storm-relative flow and some protection from dry-air intrusion. The role of the critical-layer cat's eye in helping to provide these conditions was formulated in our second hypothesis (H2). Later in the same chapter, the author describes the transition from a subtle surface signature of easterly-wave passage, to a more robust closed surface circulation:

"We observe that occasionally, especially in late summer and early fall, the amount of convection associated with a particular wave increases, and winds near the surface evolve from a typical wavy pattern of an easterly wave into a closed circulation. A tropical depression is born.” (ibid., p. 99).

We would go, of course, a step further and identify a closed cyclonic circulation in the co-moving frame as the critical ingredient for depression formation (see Appendix). The author, nonetheless, recognizes the apparent need for a closed gyre at or close to the surface, a feature which by no means is present in all waves, but clearly exists in the developing cases considered in this paper. As to why the surface gyre forms, and why it is important to genesis, we are left to speculate that (i) diabatic activation ${ }^{30}$ of the wave by deep moist convection is needed to amplify the effect of an otherwise "dry"

\footnotetext{
${ }^{30}$ It is clear from Berry and Thorncroft (2005) that the amplitude of waves emerging from West Africa is not a good indicator of future development, except near the coast.
}

critical layer ${ }^{31}(\mathrm{H} 3)$ while (ii) Ekman pumping from the tropical boundary layer is needed (or desirable, at least) to organize convection in a ring-like pattern about the proto-vortex (Eliassen and Lystad, 1977; Van Sang et al., 2008). In a rotating environment such pumping is sensitive to the radial gradient of absolute vorticity (Ooyama, 1969; Hack and Schubert, 1986). The role of boundary-layer forcing in a co-moving frame was studied in the classic paper by Shapiro (1983) for mature hurricanes. Wind speeds in the proto-vortex are considerably less, but (as in a mature system) surface drag varies quadratically with wind speed for speeds greater than a few $\mathrm{ms}^{-1}$ but less than 25 knots. Therefore, azimuthal asymmetries of BL forcing are relevant to tropical depression formation.

Perhaps the most impressive and humbling aspect of these observations is the great variety of flow configurations seen in the 55 cases selected for study. Although generally similar and tending to support the marsupial paradigm, no two events are exactly alike. Their similarities prevent us from labeling TC genesis as an entirely turbulent process, thanks to the underlying synoptic wave structure and its implications for flow kinematics when superposed on the adjacent easterly jet. In the co-moving frame, the region of closed circulation displays a preferred, non-random orientation with respect to the jet axis and its associated gradients of vorticity and moisture. The basic state represents a "coherent structure" in the lingo of contemporary GFD, just as the tropical depressions that form on it, and grow for a time within their parent waves. So the real world of TC genesis is not a story about quasi-2D turbulence nor a moist generalization thereof - not exclusively, at least. Nonetheless, differences from one event to the next compel us to recognize the disordered and variable nature of the sub-synoptic dynamics, a characteristic partly attributable to quasi-2-D turbulence but magnified by rotating 3-D-convective turbulence, as contained in the ubiquitous "vortical hot towers" (Frank and Johnson, 1969; M06). One can hardly imagine a more fascinating interactive, multiscale system in nature than this.

\footnotetext{
${ }^{31}$ Tropical waves display moist propagation speed even when not coupled to convection if background convection is present (Dunkerton and Baldwin, 1995) so we may regard the incident easterly waves as linearly moist waves prior to their diabatic activation by a proto-vortex in the critical layer. Subsequent to activation, organization of convection by the wave-vortex hybrid implies a "conditional" or phase-dependent diabatic heating (Crum and Dunkerton, 1992, 1993).
} 


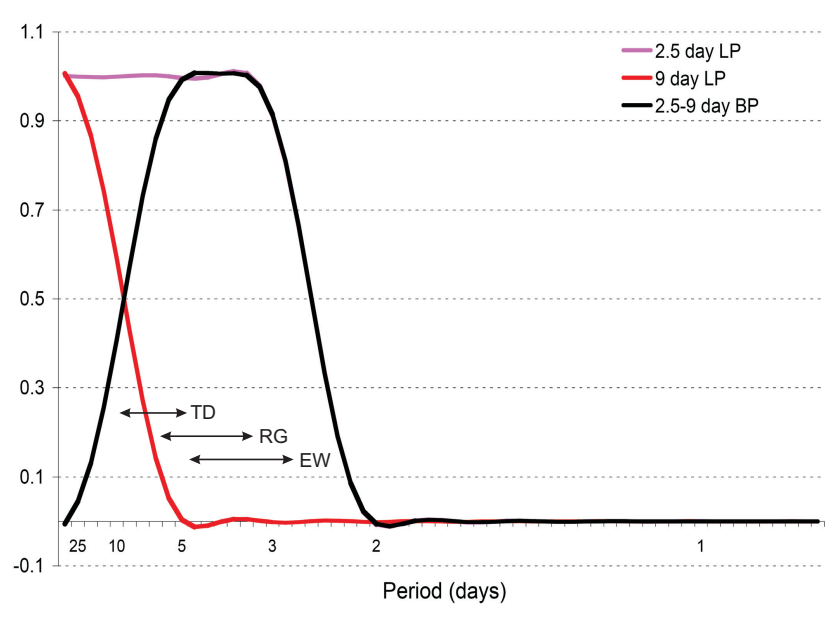

Fig. A1. Frequency response of temporal filters used in this study. A low-pass filter (purple; periods longer than $\sim 2.5$ days) describes the total flow evolution sans high-frequency component. A bandpass filter (black; periods between $\sim 2.5$ and 9 days) captures tropical waves in this frequency range for the purpose of estimating their propagation speed in Hovmöller diagrams. A low-frequency filter (red; periods longer than 9 days) provides an informal estimate of critical latitudes as seen by the waves.

\section{Appendix A}

\section{Data and methodology}

\section{A1 Datasets and filters}

The time period of interest encompasses a substantial fraction of the hurricane seasons of 1998-2001, the years when the two primary datasets used in our study overlap: (i) ERA-40 6-hourly re-analyses were used to analyze tropical waves and nearby circulation. The data are available from NCAR at multiple pressure levels in T106 spectral resolution $\left(\sim 1.125 \times 1.125^{\circ}\right)$. Properties of ERA-40 and other analyses (e.g., circulations and physical fluxes) were recently evaluated and compared by Grotjahn (2007). A 2.5-9 day bandpass filter (Doblas-Reyes and Deque, 1998) was applied to the original data to extract wave propagation signals, while a 2.5-day low-pass filtered wind field was used to define the total flow (i.e., wave + mean fields) in the vicinity of the wave. The response functions of the filters are shown in Fig. A1. It is important to note that the band-pass data supply information on wave packet structure and propagation, while the total flow (except for its high-frequency component) defines critical layers, stream function or streamlines, Lagrangian boundaries, and so forth. Low-frequency data (periods longer than 9 days) were used casually to estimate the mean flow and its critical latitudes as seen by the waves, not by individual parcels, which respond instead to the total flow sans high frequency component. (Parcel displacements are relatively insensitive to high frequency oscillations because they are inversely proportional to intrinsic frequency in linear the- ory.) (ii) Rainfall data from TRMM $3 \mathrm{~B} 42^{32}$, available every $3 \mathrm{~h}$ and having a spatial resolution of $0.25 \times 0.25^{\circ}$, were used in the study as a proxy for deep moist convection. The level 3 gridded data are convenient for this purpose but occasionally suffer gaps in coverage owing to the satellite orbit. ERA-40 re-analyses and TRMM rainfall are independent data. The time period of ERA-40 used in this study (1998-2001) derives from Stream 1 (1987-2002) which incorporates satellite data from ATOVS, SSM/I and ERS, in addition to satellite data from VTPR, TOVS and cloud motion winds used in Stream 3 (1972-1988). ERA-15 analyses for tropical waves were validated against rawinsonde data by Dunkerton and Baldwin (1995). We consider the ECMWF product to be optimum for synoptic-scale waves, particularly for the rotational component of horizontal winds in the lower and upper troposphere and (presumably) for the associated horizontal transports of moisture in the lower troposphere as analyzed in Stream 1. The divergent component is problematic in global analyses, whether from ECMWF or elsewhere, particularly when associated with deep convection. Vertical motions are not observed directly, but are obtained from a cumulus parameterization. Regions of deep convection, moreover, tend to concentrate vertical motions at the grid scale, making the resulting maximum upward velocities resolutiondependent (in such a way as to conserve approximately the total vertical flux of mass, which is driven by the cumulus scheme). Convective precipitation from TRMM is a derived product which we employ here, not for quantitative precipitation, but for geolocation of convecting features relative to the ECMWF product. It was shown by Julian (1984) that a deep cloud proxy (OLR) is correlated with upper tropospheric divergence. Needless to say, the TRMM geolocation $(\sim 25 \mathrm{~km})$ is accurate enough for the purpose at hand. We therefore regard the juxtaposition of ERA-40 and TRMM data to be a useful way of relating actual convection (an indicator of TD development) to actual waves, in the discussion to follow. Forecaster's best-track data provide a third independent source for genesis location and storm track although as we will argue, their identification of genesis time is sometimes delayed, perhaps owing to a conservative mindset ${ }^{33}$. Storm geolocation based on geostationary imagery is accurate to within the nominal $4 \mathrm{~km}$ pixel resolution at the sub-satellite point, superior to any of the other products used in our analysis. August and September were chosen because they represent the peak of the Atlantic hurricane season (Kimball and Mulekar, 2004).

\footnotetext{
${ }^{32} \mathrm{http} / / /$ disc.sci.gsfc.nasa.gov/TRMM/precipitation/trmm_intro. shtml

${ }^{33}$ The best-track (retrospective) estimate of genesis time can be earlier than the initial (real-time) estimate by as much as 2 days. Our point is that, in some cases, even the best-track estimate is late, relative to the analyzed Lagrangian criteria. For westward propagating disturbances, the temporal offset implies a longitudinal offset, which can be as large as $\sim 20^{\circ}$ in extreme cases.
} 


\section{A2 Critical latitude definition}

At the critical latitude or level, the intrinsic wave frequency goes to zero. Mean flow and wave phase speed in the direction of wave propagation are equal, i.e., $c=\mathbf{k}^{*} \mathbf{U}$, where $\mathbf{U}$ is the mean vector wind, and $\mathbf{k}^{*}$ is the unit wavevector. For zonally propagating waves, this equation can be simplified, and the critical latitude is defined as the location where $c=U$, where $U$ is the zonal component of mean vector wind.

\section{A3 Wave tracking algorithm}

For quasi-monochromic easterly waves, a semi-automated algorithm was developed in order to track the incident waves and to calculate their zonal phase speeds. The tracking algorithm is based on a Hovmöller diagram of 2.5-9 day bandpass meridional wind along the genesis latitude as identified in the best-track data. The exact choice of latitude is relatively unimportant, as nearby grid points give similar results. What is more important is the choice of genesis time, as noted below. First, the location of meridional wind anomalies was determined at genesis time within $\sim 16.9^{\circ}$ ( 15 grid points) on both sides of the genesis longitude $(x)$. Anomalies were then traced backward in time $(t)$ from this point for $72 \mathrm{~h}$. For such an anomaly to be acceptable it was required that the local maximum and minimum wind perturbations last at least $12 \mathrm{~h}$. To identify wind anomalies which are presumably most directly related to genesis, the time series of maximum and minimum perturbations nearest in $x$ surrounding the genesis point were selected, and two phase speeds, $c^{+}$and $c^{-}$, derived based on a linear regression ( $t$ upon $x$ ) of the areas (in the $x, t$ plane) of southerly and northerly wind perturbations, respectively, exceeding $1 \mathrm{~ms}^{-1}$ in absolute value. Two additional estimates of phase speed were likewise obtained for the period from $-48 \mathrm{~h}$ to $+24 \mathrm{~h}$. This procedure for quantifying wave characteristics semi-automatically is similar to that shown in Fig. 11 of Dunkerton and Baldwin (1995). The algorithm as described above was found superior to an alternative procedure following only the local maxima and minima of $v^{\prime}$ instead of the full anomaly area. If the phase speeds as determined by the tracking algorithm were found to represent adequately the observed wave propagation, as indicated by the slopes of meridional wind anomalies in the Hovmöller diagrams, their average $\left(c_{m}\right)$ was used to define the incident wave phase speed at this altitude. Note that we do not mix adjacent altitudes for this purpose.

Our default was to select phase speeds based on a time period $72 \mathrm{~h}$ before genesis. It is preferable to exclude data after genesis because parent waves are often changed by the tropical depression, and new waves excited. In some cases the pre-genesis lifespan of a parent wave is exceptionally short. We allow, therefore, and only where necessary, an alternative definition of phase speed based on data extending from $48 \mathrm{~h}$ before best-track genesis to $24 \mathrm{~h}$ after genesis. This alternative definition was adopted if and only if one or more of the default values of phase speed were unsuitable, as determined by a visual evaluation of the goodness of fit. It should be noted that best-track data do not necessarily indicate the exact moment of genesis because the time chosen for the first advisory is based on a human decision. The subjective nature of the operational procedure will become evident in one of the cases highlighted below (Chris 2000) wherein a depression-strength closed gyre in the co-moving frame $\sim 30 \mathrm{~h}$ prior to "genesis" in the Earth-relative frame.

\section{A4 Vertical structure}

Prior to genesis, easterly waves are generally confined to the lower troposphere at or below $\sim 500 \mathrm{hPa}$, with equivalent barotropic structure or slight vertical phase tilt. In some cases the phase tilt in the lower troposphere is observed to change with time during the storm development stage. It is instructive therefore to examine their propagation at two representative levels, 850 and $600 \mathrm{hPa}$. In most cases analyzed, the same wave packet could be identified at both levels with similar propagation characteristics. Their averaged phase speeds on each level ( $c_{m}$ as defined above) generally differ - usually by a small amount, but sometimes significantly so in order to handle the latter contingency it is preferable to use the phase speed at a particular level when analyzing data at that level, rather than a blend of phase speeds from different levels to characterize the incident wave propagation. The vertical structure of tropical waves participating in TC genesis is an interesting part of the marsupial story, and its evolution throughout the depth of the troposphere has several typical characteristics. The transition of wave structure from a trapped LT disturbance to deep "first baroclinic mode" structure immediately before genesis, subsequent vortex shedding in the lower troposphere and radiation of new waves (if any) have been identified using multi-dimensional visualization software from Pixotec. Some aspects of vertical structure are highlighted in Sect. 4.

\section{A5 Translating coordinate system}

A key element in the analysis is the depiction of horizontal flow in a co-moving frame of reference, i.e., moving westward with the parent easterly wave. It should be clear from the opening remarks of Sect. 2 that the critical layer of a traveling wave becomes stationary when viewed in a coordinate system moving horizontally at the phase speed of the wave. The simplest case is a single wave with constant phase speed that is uniform in time and space; the translation speed is then a constant, and whatever unsteadiness remains in the wave field is attributable to amplitude evolution or structural change, rather than phase propagation. An interesting extension is to have a modest spread of frequencies about a central frequency, with the possibility of horizontal dispersion, forming a wave packet with group propagation differing from the phase propagation (for a general discussion of 
waves in fluids see Lighthill, 1978; for examples of group propagation in tropical waves see Liebmann and Hendon, 1990; Dunkerton and Baldwin, 1995). As long as the packet contains multiple phases in physical space, as implied by a modest spread in spectral space, it is appropriate to translate the coordinate system at the dominant phase speed, usually resulting in a non-zero zonal group propagation as viewed in the co-moving frame. Cases of TC genesis involving the intersection of two or more waves with substantially different phase speeds, as sometimes observed in the western tropical and subtropical Pacific, are outside the scope of this paper and will be discussed elsewhere in this series.

The purpose of translating to a co-moving frame is to render wave phases approximately stationary, in order to minimize the temporal evolution of the flow pattern. In steady flow, streamlines are equivalent to trajectories (Holton, 2004). If the flow pattern consists of a steady mean flow and quasi-monochromatic wave, streamlines in a coordinate system moving at the phase speed of the wave may then be used as a surrogate for parcel trajectories. Allowing for a modest dispersion or other kind of amplitude evolution, the temporally evolving streamlines still provide a useful, albeit approximate, depiction of particle paths. For example, if a wave packet in the co-moving frame were to creep slowly eastward, forming new phases to the east while old ones decay to the west (resembling surface waves on water), parcel motions near the longitude of interest could be anticipated simply by waiting for the packet to arrive at that longitude.

Similar reasoning applies to an isolated region of enhanced vorticity, as might be found at the peak amplitude of a wave packet, or in a single wave phase where diabatic amplification is exceptionally strong for whatever reason. Figure 3 illustrates streamlines of a point vortex, with cyclonic circulation, translating to the west (panel a), stationary (b) or translating to the east (c) relative to the fluid. For sake of illustration we are not concerned with why the vortex translates, other than to assume that some external agent is responsible for the translation (since left to itself, the vortex would be subject to magnus and advective forces). The vortexinduced flow is a function of $x-C_{p} t-$ assumed steady, that is, in a frame of reference moving with the vortex at its velocity $C_{p} \hat{1}$, while the imposed background flow velocity is $U_{b} \hat{1}$. The kinematic parcel displacement field is an intrinsic fluid-dynamical property and therefore depends on the difference $C_{p}-U_{b}$ and not on the terms individually. The vortex can be imagined to induce parcel displacements which decrease in amplitude with increasing distance from the vortex center. When the vortex translates zonally in one direction or the other, parcels experience either a temporary influence of the passing vortex (far from center) or are permanently trapped in a vortex-induced gyre (close to center). A bounding streamline separates these outer and inner regions, and a stagnation point exists where vortex and ambient flows cancel. The stagnation point defines the dividing streamline; it is also a saddle point of stream function in a horizontally non-divergent flow. This flow configuration is the point-vortex analogue of the discontinuous Lagrangian mean flow associated with a steady wave critical layer described in Section 2a. What is missing ${ }^{34}$ from the pointvortex flow in Fig. 3 (panel b in particular) that was present in the critical layer of Fig. 1 is the latitudinal shear (for constant shear, a parabolic $y$-profile of ambient stream function) that suppresses the meridional component of parcel displacement at large distances from the centerline. Owing to latitudinal shear, the cat's eye has a bounding streamline, whereas the stationary vortex in a resting flow does not. (A train of cat's eyes can be formed by placing equally spaced point vortices along an isotach in parallel shear flow, as shown in Fig. 2.10.1 of Pozrikidis, 1997.) Panels (a) and (c) demonstrate that trapping of parcels nevertheless can occur close to vortex center, without shear, because the nearby influence of the vortex (a singular point) is stronger than that of the vortex-relative background flow $U_{b}$. The streamline patterns in Fig. 3 are steady in a frame of reference moving with velocity $C_{p} \hat{1}$ matching that of the vortex in the same way that the streamline pattern of a nonlinear wave critical layer is steady in a frame of reference moving with the wave at its phase velocity $C_{p} \hat{1}$. In the properly translating frame, streamlines are therefore equivalent to trajectories.

We may also interpret the panels of Fig. 3 as depicting the instantaneous view of streamlines obtained by observers in different reference frames moving at velocity $C_{r} \hat{1}-$ whether "translating properly" or not. Note that proper translation requires $C_{r}=C_{p}$ whereupon the vortex-induced flow becomes a function of $x_{r} \equiv x-C_{r} t=x-C_{p} t$ and the explicit dependence on $t$ is eliminated. For example, the viewpoint of panel (c) is obtained in the Earth-relative frame for a vortex moving westward in this frame. It is important to note that only one of these viewpoints is "proper" for a particular $U_{b}$ in the sense that streamlines are equivalent to trajectories only in the frame moving with the vortex, because the vortex-induced flow is steady in this frame only ${ }^{35}$. For

\footnotetext{
${ }^{34}$ The critical layer and point vortex also differ in their vorticity distributions and the horizontal flows induced (via PV inversion) by the distributions of vorticity. The steady asymptotic critical layer consists of a constant background vorticity gradient plus an eastwest train of cat's eyes with homogenized PV within; the point vortex consists of an isolated "PV charge" and constant background velocity (the constant cannot be obtained by $\mathrm{PV}$ inversion). When the vorticity of the point vortex is distributed monotonically over a finite circular area, a dividing streamline exists if and only if the background velocity does not exceed the maximum wind at the edge of the vorticity distribution.

${ }^{35}$ We should therefore qualify Emanuel's (2005, p. 127) recommendation that streamlines - owing to their non-uniqueness - not be used to locate a storm center because, as shown in this paper, a translation speed determined by the parent wave provides a uniquely "proper" view of translating (possibly closed) streamlines that agree with parcel trajectories over a time span of a few days and provide an optimum view of the horizontal transport of adiabatic invariants.
} 
a westward-moving vortex, the stationary (Earth-relative) frame gives a misleading picture of flow kinematics. Figure 3 demonstrates, among other things, that the proper choice of reference frame is essential in order to understand flow kinematics in the neighborhood of the vortex (Ide et al., 2002; $\mathrm{K}$. Ide, personal communication). The number of closed contours and the sense in which the vortex "connects" to the exterior flow (i.e., at the nearest saddle point) obviously depend on the choice of frame. The frame of reference is therefore important for an optimal view of horizontal transport as well. In the context of Atlantic easterly waves we infer, for instance, that if environmental air were moist in one direction (e.g., near the ITCZ) but dry in another (e.g., near the equator), the orientation of the connection might significantly affect diabatic processes regulating the subsequent vortex development (H2). Entrainment of absolute vorticity would be affected similarly in the presence of a mean vorticity gradient (H1). Note also that the translating views of a point vortex introduce a north-south asymmetry, reminiscent of actual easterly wave troughs, that was not present in the idealized critical layer of a shear flow in Fig. 1. The asymmetric structure and existence of a separatrix on one or both sides of the cat's eye proves to be a characteristic feature in many observed genesis events, including western Pacific events (Edson and Lander, 2006).

\section{A6 Horizontal flow potentials and the choice of "gauge"}

For synoptic flows, even in the tropics, the horizontally rotational part is much larger than the horizontally divergent part, with certain exceptions, so that streamlines can be approximated by a stream function using the Helmholtz decomposition of the horizontal velocity vector $\mathbf{U}$. Crucial to our analysis in Sect. 3 is that the stream function and velocity potential, like any other potential, are known only up to a "gauge", i.e., a function whose horizontal Laplacian is zero and therefore makes no contribution to relative vorticity or divergence, which are Galilean invariant; that is, invariant to a uniform horizontal translation of the coordinate system, as contemplated here. Remarkably, there are an infinite number of stream functions (velocity potentials) that are perfectly consistent with the actual vorticity (divergence). For a quasi-monochromatic wave superposed on a steady mean flow, however, only one choice of translation speed, or gauge, makes sense: viz., that corresponding to a uniform horizontal translation at the phase speed of the wave ${ }^{36}$. By viewing

This comment applies equally to genesis and mature stages, although in a fully developed storm the time span is considerably shorter.

${ }^{36}$ A more general construction of Lagrangian boundaries from an Eulerian diagnostic is possible in 2-D and 3-D flows: for some recent ideas see Haller and Yuan (2000) and Green et al. (2006) respectively. By contrast, a local "wave gauge" such as ours is designed to be optimum in one region but not in another, depending on the local wave spectrum. It nonetheless works well for the quasi- streamlines in a frame of reference moving with the wave (the "co-moving" frame) we are able to visualize the structure of recirculation regions, their formation and subsequent modification by diabatic vortices, together with the evolution of potential vorticity, moist entropy and other adiabatic invariants in such regions. It should be noted that the although the proper gauge for quasi-monochromatic waves is structurally simple, it is not a trivial constant. For zonally propagating waves, the gauge function varies linearly in latitude, and therefore always changes the stream function pattern, unless zero everywhere (i.e., no translation).

Depending on whether the reader has a degree in physics or meteorology (or both), two analogies may be offered for clarification. One is from electromagnetism: the familiar equations of Maxwell, and the introduction of potential functions to calculate the remote influence of charges that evolve in time. The simplest choice of potential, or Coulomb gauge, is not the best choice for this equation set, because it implies (or seems to imply, at first sight) instantaneous action at a distance: given a certain charge at some point in space, the entire space is seemingly affected at once by the charge, and its time variation. This inference violates the theory of special relativity, overlooking the finite speed of electromagnetic wave propagation, on which the theory is based, as implied by the equations themselves ${ }^{37}$. An alternative potential, or Lorenz gauge - which is also Lorentz invariant ${ }^{38}$ - correctly accounts for the finite propagation speed by building a temporal part into the definition of the potential function - the so-called "retarded potential". This choice of gauge is not obvious from the single equation for electric field induced by a charge, but turns out to be the preferred choice for the whole set of equations; in particular, for the radiation of electromagnetic waves from time-varying distributions of charge. In our case, the proper choice of gauge - a function that converts the stream function into something appropriate for visualizing the Lagrangian flow - is not obvious from a Helmholtz decomposition of the Eulerian flow in a resting frame. Its definition is motivated by the inclusion of a quasimonochromatic wave in the problem, if the phase speed of this wave is known a priori.

monochromatic cases considered here, aided by the fact that we are targeting a single genesis point in space-time.

${ }^{37}$ The inference is incorrect, and with extra effort (Brill and Goodman, 1967) the Coulomb gauge can be shown to be consistent with the theory of special relativity, a result more easily obtained if the Lorenz gauge is adopted in the first place. It is remarkable though overlooked initially that Einstein's theory of special relativity is contained in Maxwell's equations, insofar as the electric and magnetic permittivities, whose inverse product defines the speed of light squared, are constants independent of the choice of coordinate system, whether moving or not. The moral of this story, and the present one, is that the laws of physics do not change with a coordinate transformation, but some pathways to enlightenment are considerably shorter than others!

${ }^{38}$ http://en.wikipedia.org/wiki/Lorenz_gauge_condition 
A second and more familiar analogy is the popular "storm relative velocity" used in the depiction and interpretation of Doppler radar reflectivity from precipitation cells embedded in mesoscale (convective or stratiform) systems that move horizontally with the synoptic-scale flow. By translating to a co-moving frame defined by motion of the storm system as a whole, it is possible to visualize mesocyclones (important for tornado genesis) and other circulation features associated with the system. The ingredients in this situation are analogous to those of our translating tropical wave critical layer and its incipient tropical depression: a storm system that is steered at more or less constant speed by the environmental flow, evolving slowly in a frame of reference moving with the flow. Use of storm relative velocity is a simple way to minimize the translational evolution of the pattern of precipitation cells while retaining the evolution of the cells within the translating pattern.

\section{Appendix B}

\section{Additional cases highlighted}

Map sequences for the remaining storms highlighted in Fig. 4 are discussed in this Appendix, extending the marsupial paradigm to the west Atlantic, Gulf of Mexico and central Pacific.

\section{B1 West Atlantic: tropical storm Chris}

A similar story as Debby may be told regarding the birth of Chris, but with an odd twist: closed gyres are evident at both levels $12-30 \mathrm{~h}$ prior to the best-track genesis time, as shown in Figs. B1 and B2, but by the time the first advisory was issued, the streamlines (and stream function contours) opened up. The genesis location is consistent with that expected from westward translation of the closed gyres from the time of their disappearance. A principal separatrix existed 12$30 \mathrm{~h}$ before genesis (just to the north of the closed gyre at either level) but is either gone or displaced northward out of the picture by the time genesis was officially declared. A secondary saddle point far to the WSW is present throughout the sequence at either level. It seems odd that a closed gyre does not appear around the best-track genesis point, even though it was present a short time before. A possible explanation is that ERA-40 data do not fully resolve the horizontal structure of the tropical depression. Note however that the critical latitude seen by the waves (red curve in final panel; this quantity is based on 9-day low-frequency data and changes little in $30 \mathrm{~h}$ ) together with the analyzed trough axis pinpoints the best-track genesis location to within $\sim 100 \mathrm{~km}-$ as observed at one or both levels in each of the five storms highlighted here. The silver lining in this event may be that we are able to detect a depression-strength closed gyre more than a day in advance of the official time, simply by examining streamlines in the co-moving frame. A key consideration for operational purposes is that for this technique to be useful in real time, the easterly wave must be identifiable sufficiently in advance: identifiable, that is, not only before the official genesis time (since the depression at this time would be identified anyway without knowledge of the translating streamlines) but before an earlier point in time when we wish to issue a forecast in advance of the first. In so many words, the co-moving frame needs to be determined ahead of time. Little would be gained by issuing a forecast at the genesis time based on wave information accumulated up to that point; such knowledge is needed well in advance of the official time. With this caveat, an earlier identification of tropical depressions seems possible in cases like Chris.

Streamlines in the resting frame once again are almost useless for this system, as there are no closed streamlines anywhere (Fig. B3). The best indication of possible development might have been inferred from a trough extending from SW to NE, a tilted version of the "right-side-up" omega pattern of an isolated vortex in the resting frame (Fig. 3). As in Debby, the translating but not resting streamlines provide a reasonably consistent picture of the containment of cyclonic vorticity by a closed gyre (except for its disappearance in the analyses just prior to genesis time). The Okubo-Weiss parameter is shown in Fig. B2 at the $600 \mathrm{hPa}$ level for the genesis sequence. This quantity highlights regions of strong rotational flow (curvature vorticity), while suppressing the display of shear vorticity in regions of strong shearing deformation (third term of Sect. 4.1) flanking the jet axis ${ }^{39}$. At both levels the center of the cyclonic OW anomaly coincides almost perfectly with the best-track genesis location. A disadvantage of this quantity is that anticyclonic regions are highlighted equally. In general, we expect that OW will be useful for pinpointing genesis, in situations where an elongated vortex strip rolls up into one or more closed vortices, by isolating the curved regions of flow at the expense of the elongated features (viz., the vortex strip that caused roll-up in the first place). Perhaps the most important conclusion to draw from the OW parameter inside the translating gyre is that the flow within is relatively free of strain and shear (McWilliams, 1984), favoring nominally the formation of smaller proto-vortical structures within.

With regard to saturation fraction (Fig. B4) and precipitation (Fig. B5) similar conclusions may be inferred as in the previous case: convective precipitation moistens the column along an axis extending SW-ward from the gyre center while advection carries this moisture to the NE, allowing some to escape the domain while the remaining moisture is contained in the northern half of the gyre. It is difficult to evaluate the containment effect since precipitation is rather constant near the genesis location throughout the sequence. The distribution of precipitation in relation to vorticity is interesting: an

\footnotetext{
${ }^{39}$ Curvature vorticity is useful for this purpose if calculated in the co-moving frame, not the resting one.
} 


$$
\text { Chris: Zeta (2.5-day LP) }
$$
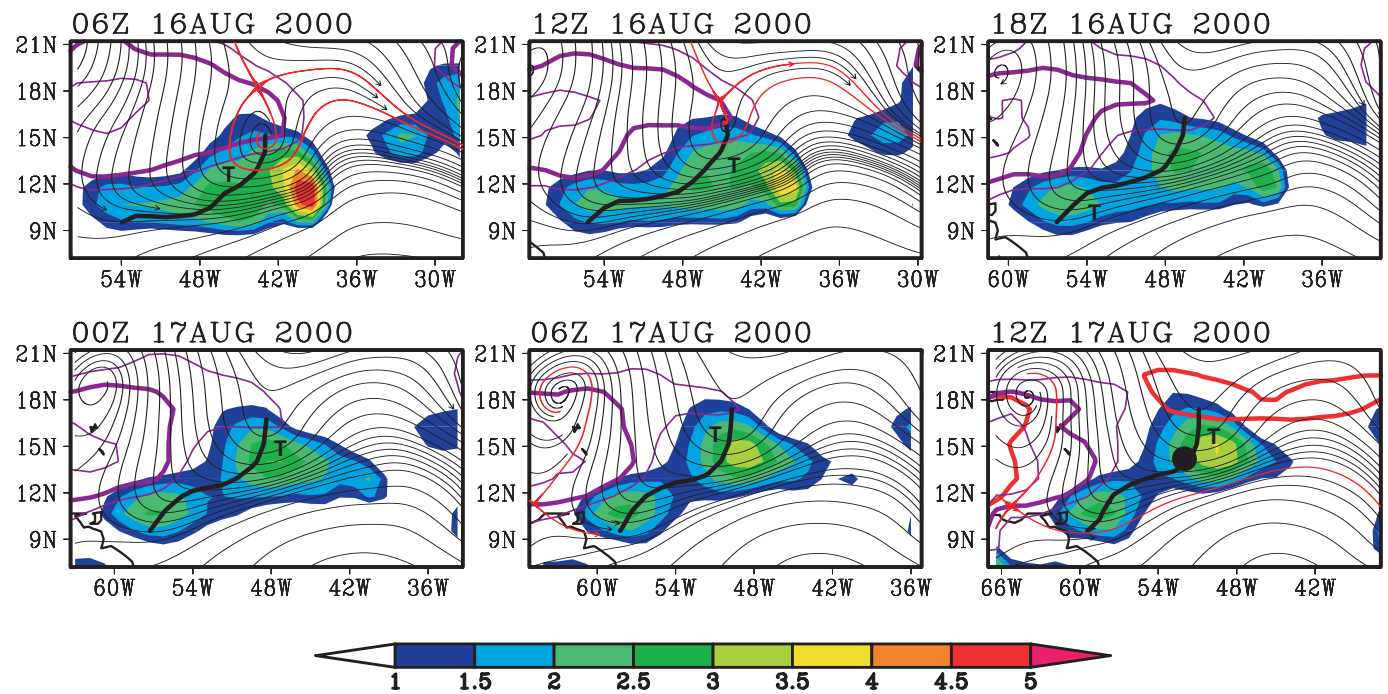

Fig. B1. Streamlines of horizontal (rotational + divergent) flow at $850 \mathrm{hPa}$, as seen in ERA-40 data, for six consecutive analyses leading up to the best-track genesis time of Tropical Storm Chris (2000). Shading indicates relative vorticity (units: $10^{-5} \mathrm{~s}^{-1}$ ). The sequence of frames translates westward at the zonal propagation speed of the parent wave at $850 \mathrm{hPa}$ as estimated from the Hovmöller method $\left(-9.1 \pm 1.1 \mathrm{~ms}^{-1}\right)$ and streamlines are calculated and displayed in the co-moving frame; note that relative vorticity itself is invariant with respect to the translation. Isopleths of zero relative zonal flow are shown (purple) together with their uncertainty. In the final panel of the sequence the critical latitude of the parent wave is also indicated (red) corresponding to isopleths of zero relative zonal flow in low-frequency data with periods longer than 9 days. The wave's trough axis is shown for reference (black). The genesis location is indicated by the black dot in the final panel.

$$
\text { Chris: OW (2.5-day LP) }
$$
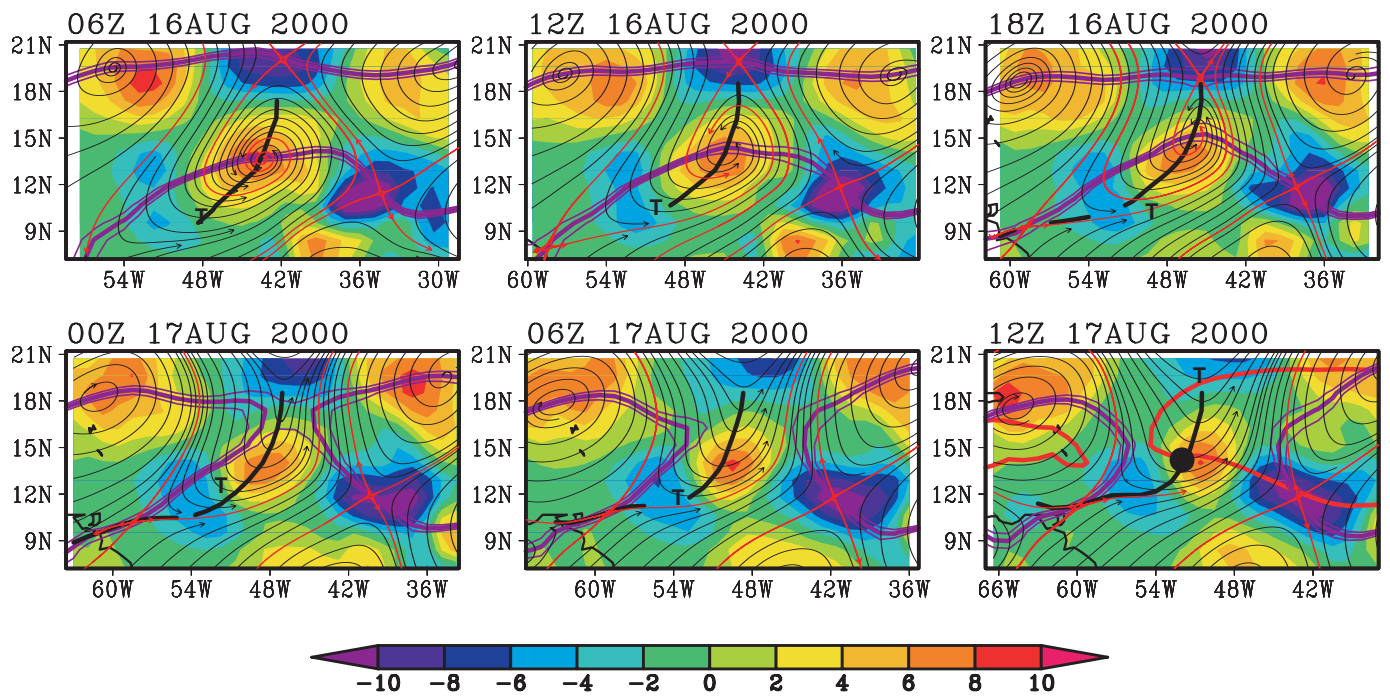

Fig. B2. Stream function of horizontal flow for the genesis sequence of Tropical Storm Chris as in Fig. 23, but at $600 \mathrm{hPa}$. Shading indicates the Okubo-Weiss parameter (units: $10^{-10} \mathrm{~s}^{-2}$ ) as defined in Eq. (2). This quantity, like vorticity, is invariant with respect to translation, therefore identical in co-moving and resting frames. The sequence of frames translates westward at the zonal propagation speed of the parent wave at $600 \mathrm{hPa}\left(-8.5 \pm 0.5 \mathrm{~ms}^{-1}\right)$ and stream function is calculated and displayed in the co-moving frame. 
Chris: Zeta (2.5-day LP, resting)

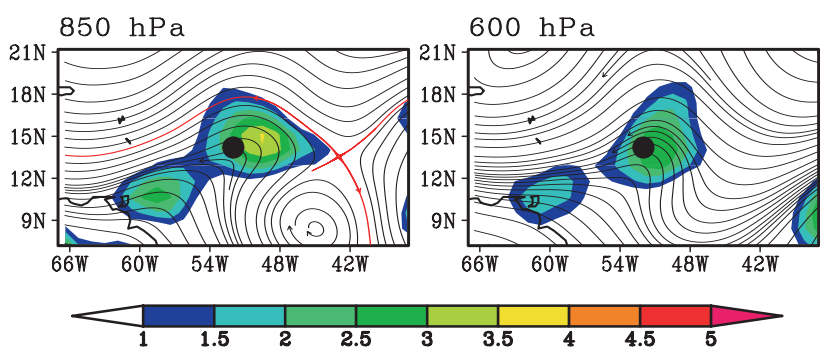

Fig. B3. Streamlines of horizontal flow at (a) $850 \mathrm{hPa}$ and (b) $600 \mathrm{hPa}$ at the genesis time of Tropical Storm Chris, in the resting frame. Shading indicates relative vorticity (units: $10^{-5} \mathrm{~s}^{-1}$ ) which is invariant with respect to translation, therefore identical in co-moving and resting frames (cp. final panel of Figs. 23, 24).

apparent spatial correlation with shear vorticity (but not OW) early in the sequence, oriented to the SW of center (following the trough axis: black curve) followed by precipitation at the center only (correlated with OW, but not shear vorticity). There may have been some initial tendency for frontogenesis in the distended flow along the axis to the SW, possibly triggering the deep convection, but as genesis time approached this convective line was replaced by rotating convection, or convection tied to the rotating flow near the genesis location.

\section{B2 Gulf of Mexico: tropical storm Beryl}

Tropical storm Beryl demonstrates that westward propagating anomalies of meridional velocity may influence TC genesis as far north as the Gulf of Mexico. In this case, however, the incident wave was relatively weak compared to that of the previous examples, while propagating into a more complex flow pattern in such a way as to enhance the prevailing vortical flow, resulting in genesis. The full sequence of events in the translating frame is shown in Figs. B6 and B7 at the 850 and $600 \mathrm{hPa}$ levels, respectively. The viewpoint in the resting frame at genesis time only is shown at these two levels in Fig. B8. Since the translation speed is relatively small in this case, compared to the previous examples, either reference frame (translating or resting) is able to capture the closed gyre and correctly anticipate the genesis location and time. Close inspection of Fig. B8, however, once again reveals a slightly misaligned streamline pattern, implying a first-order vorticity advection that does not exist in the comoving frame. As noted in Debby, the Earth-relative flow is responsible, in part, for horizontal translation of the pattern a detail that is irrelevant to the internal dynamics and tends to obscure the in situ evolution. One could argue that a proper frame of reference is superior, even in cases with smaller translation speed, like Beryl ${ }^{40}$. In the co-moving frame the

\footnotetext{
${ }^{40}$ The Earth-relative frame, however, may be important for evaluation of surface fluxes, as elaborated in our response to an Interac-
}

principal saddle point exists to the NE of the closed gyre and a secondary saddle is located to the SW. A second and fairly significant gyre exists in the SW corner of the figures, making the entire pattern look like a critical layer aligned from SW to NE, tilted $\sim 45^{\circ}$ relative to a latitude circle. The incident wave alone cannot explain this pattern. Without further analysis (outside the scope of this paper) it is difficult to say how much of the flow kinematics should be attributed to the prevailing flow and to what extent this flow was altered by the incident wave, other than to amplify the "pocket" of cyclonic vorticity that eventually became a closed circulation. The transition from a linear vorticity pattern to a more circular one at the end of the sequence suggests that the incident wave was instrumental not only in enhancing the local vorticity maximum but also in altering the flow topology around this maximum in such a way as to produce a much larger region of closed recirculating flow than was present initially. This contribution is apparent at both levels. The prevailing distributions of saturation fraction (Fig. B9) and precipitation (Fig. B10) display a persistent ribbon, or in Newell's terminology (Zhu and Newell, 1999) a "river" of moisture along the same SW-NE axis as the prevailing critical layer. As with vorticity, the shape of the moist convecting region is altered from an extensive linear pattern (aligned from SW to NE) to a more confined circular patch - all of this before best-track genesis. We are evidently dealing with a more complex situation than imagined in our simple scenario outlined in Sect. 2 and as illustrated by the preceding examples in the central Atlantic. Nonetheless, the gyre circulation in this event displays a tantalizing relationship to the prevailing moisture and convection that was also seen in the simpler cases highlighted above: the closed gyre evidently forms at, or close to, a boundary between relatively wet and dry air. By the end of the sequence (genesis time) the moisture and its associated convection have engulfed most of the enclosed circulation. Is there something about the initial moisture gradient that is instrumental either in the formation of a closed circulation pattern or in the genesis to follow? The example of Fabio (Sect. 3) from the eastern Pacific, provided another illustration of this intriguing relationship and piques our curiosity further.

\section{B3 Central Pacific: super typhoon Shanshan}

The sequence of events prior to the birth of Shanshan illustrates vortex roll-up similar to that of Fabio, but more clearly. Also illustrated clearly is a vertical phase tilt of the parent wave that changes in time, towards a vertically aligned arrangement at genesis time. The $850 \mathrm{hPa}$ flow, shown in Fig. B11, consists of a band of vorticity that acquires a more circular symmetry as time proceeds. It appears from Fig. $4 \mathrm{i}$, $\mathrm{j}$ that a weak wave propagating at $850 \mathrm{hPa}$ from the east intersected a stronger wave (or instability?) to the west of

tive Comment. 


$$
\text { Chris: SF (2.5-day LP) }
$$
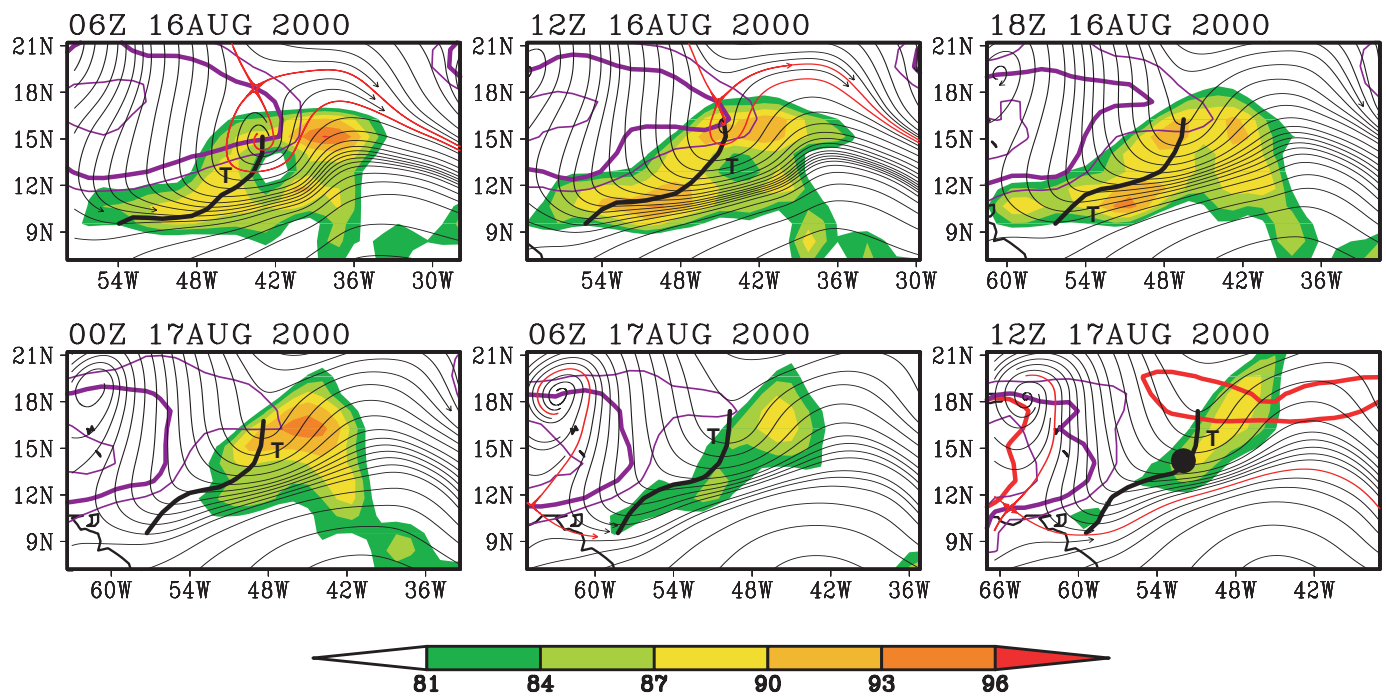

Fig. B4. Streamlines of horizontal flow at $850 \mathrm{hPa}$ for the genesis sequence of Tropical Storm Chris as in Fig. 23, with high values of ERA-40 saturation fraction indicated by shading (units: percent). The sequence of frames translates westward at the zonal propagation speed of the parent wave at $850 \mathrm{hPa}\left(-9.1 \pm 1.1 \mathrm{~ms}^{-1}\right)$ and streamlines are calculated and displayed in the co-moving frame.

$$
\text { Chris: TRMM (2.5-day LP) }
$$
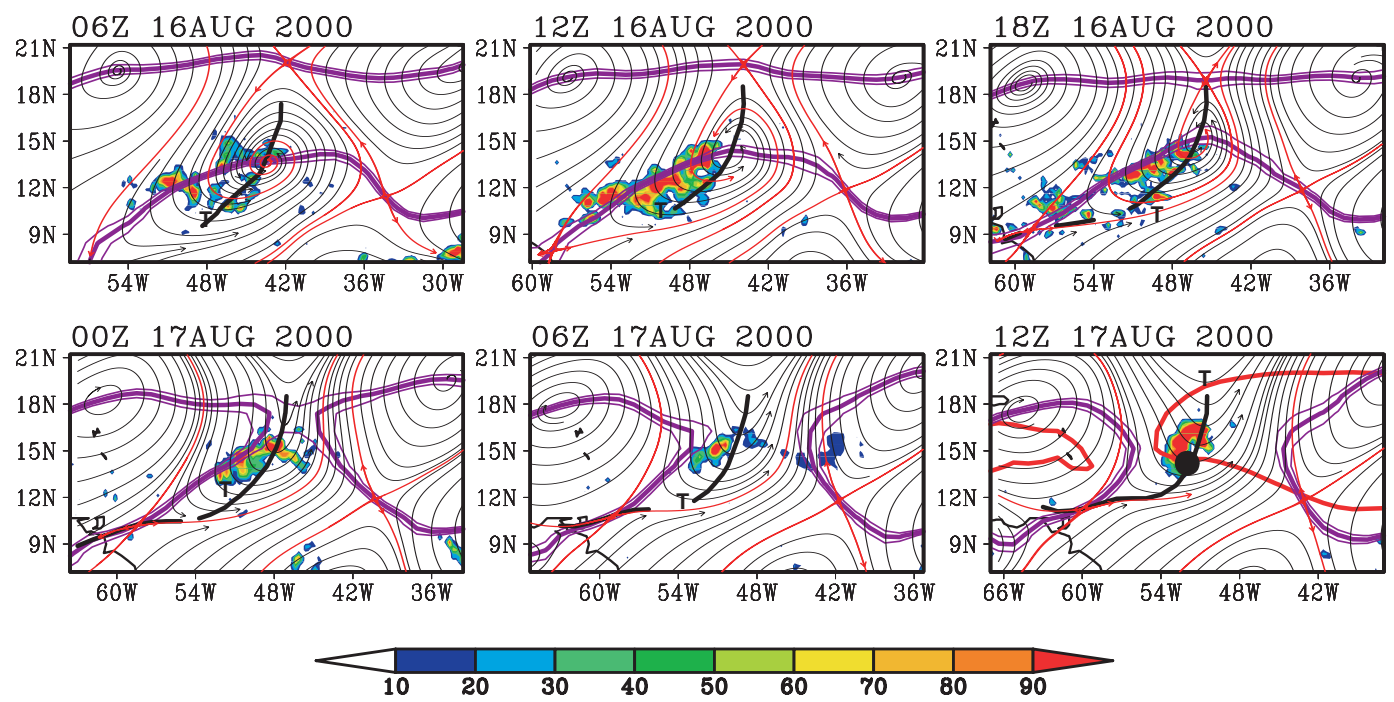

Fig. B5. Streamlines of horizontal flow at $600 \mathrm{hPa}$ for the genesis sequence of Tropical Storm Chris as in Fig. 24, with high values of TRMM 3-h accumulated precipitation indicated by shading (units: $\mathrm{mm} /$ day). The sequence of frames translates westward at the zonal propagation speed of the parent wave at $600 \mathrm{hPa}\left(-8.5 \pm 0.5 \mathrm{~ms}^{-1}\right)$ and streamlines are calculated and displayed in the co-moving frame.

the genesis location, also with westward phase propagation, but apparent eastward group propagation at $600 \mathrm{hPa}$. The proper choice of translation speed is more difficult to determine in this case compared to the preceding examples. As expected from a relatively fast westward translation, the pattern of $600 \mathrm{hPa}$ streamlines in the co-moving frame, shown in Fig. B12, looks markedly different than either the corresponding pattern at $850 \mathrm{hPa}$ (with slower translation speed) or those at either level in the resting frame (as shown in Fig. B13 for the genesis time only). The translating and resting patterns at $850 \mathrm{hPa}$ are similar because the translation speed is slow - the slowest of any of the examples thus far. 


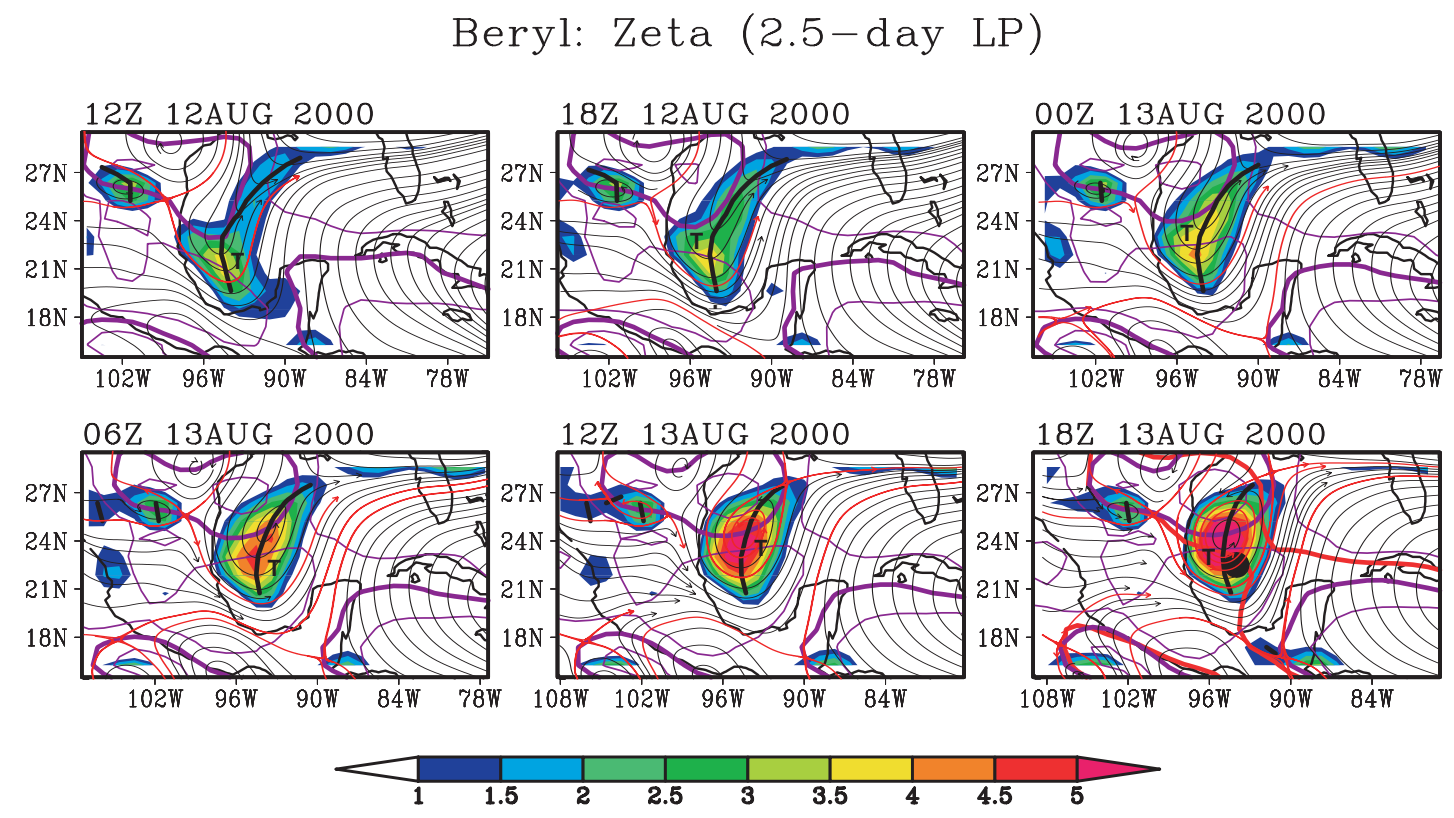

Fig. B6. Streamlines of horizontal (rotational + divergent) flow at $850 \mathrm{hPa}$, as seen in ERA-40 data, for six consecutive analyses leading up to the best-track genesis time of Tropical Storm Beryl (2000). Shading indicates relative vorticity (units: $10^{-5} \mathrm{~s}^{-1}$ ). The sequence of frames translates westward at the zonal propagation speed of the parent wave at $850 \mathrm{hPa}$ as estimated from the Hovmöller method $\left(-3.8 \pm 2.8 \mathrm{~ms}^{-1}\right)$ and streamlines are calculated and displayed in the co-moving frame; note that relative vorticity itself is invariant with respect to the translation. Isopleths of zero relative zonal flow are shown (purple) together with their uncertainty. In the final panel of the sequence the critical latitude of the parent wave is also indicated (red) corresponding to isopleths of zero relative zonal flow in low-frequency data with periods longer than 9 days. The wave's trough axis is shown for reference (black). The genesis location is indicated by the black dot in the final panel.

\section{Beryl: Zeta (2.5-day LP)}
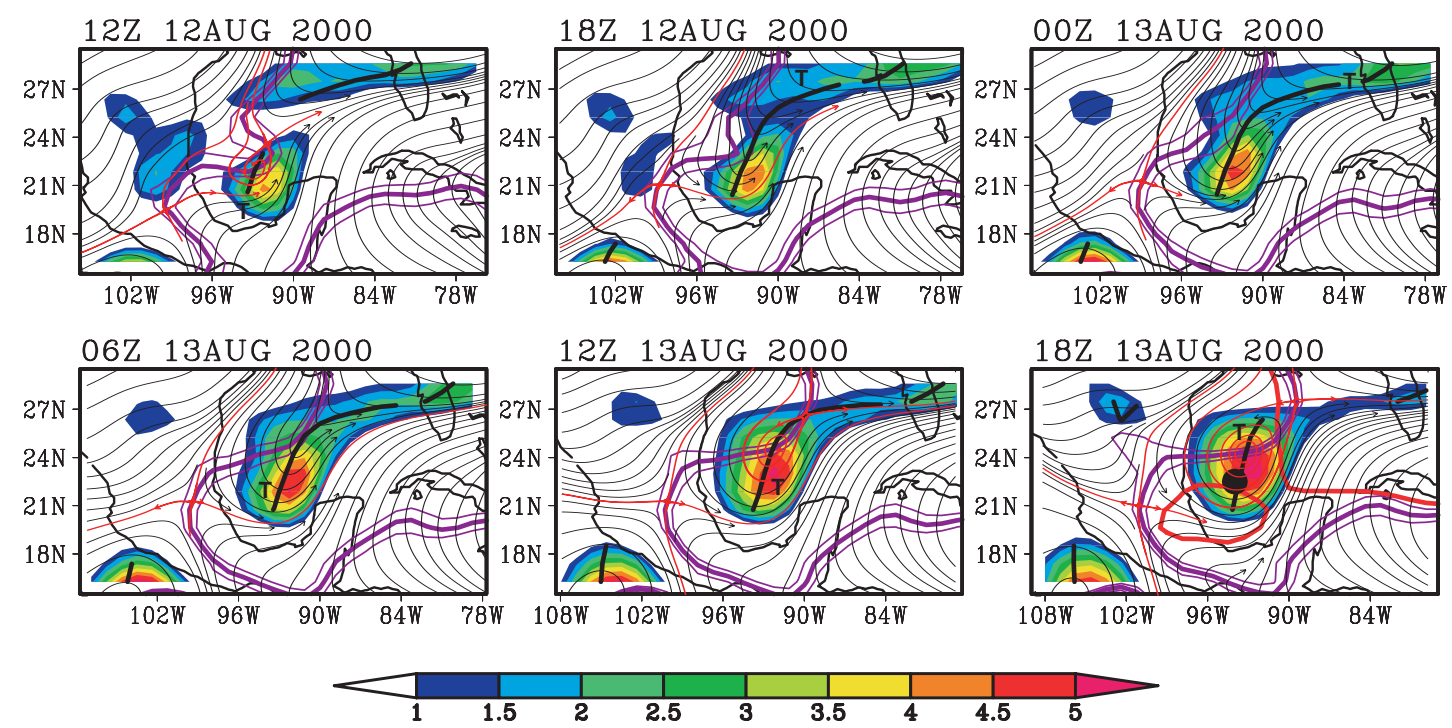

Fig. B7. Streamlines of horizontal flow for the genesis sequence of Tropical Storm Beryl as in Fig. 28, but at $600 \mathrm{hPa}$. Shading indicates relative vorticity (units: $10^{-5} \mathrm{~s}^{-1}$ ). The sequence of frames translates westward at the zonal propagation speed of the parent wave at $600 \mathrm{hPa}$ $\left(-3.1 \pm 0.8 \mathrm{~ms}^{-1}\right)$ and streamlines are calculated and displayed in the co-moving frame. 
Beryl: Zeta (2.5-day LP, resting)

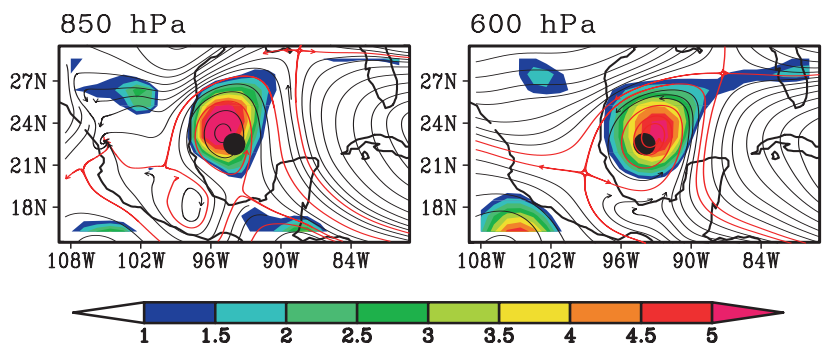

Fig. B8. Streamlines of horizontal flow at (a) $850 \mathrm{hPa}$ and (b) $600 \mathrm{hPa}$ at the genesis time of Tropical Storm Beryl, in the resting frame. Shading indicates relative vorticity (units: $10^{-5} \mathrm{~s}^{-1}$ ) which is invariant with respect to translation, therefore identical in co-moving and resting frames (cp. final panels of Figs. 28, 29).

Either choice, translating or resting, yields a closed gyre (although the gyre at $600 \mathrm{hPa}$ is much larger in the co-moving frame) and the relationship of streamlines and vorticity contours is plausible either way. The deciding factor at $600 \mathrm{hPa}$ is the existence of a second gyre near $165^{\circ} \mathrm{E}$ that is absent in the resting frame. In the left half of Fig. B12f we see the familiar cat's-eye pattern aligned in the zonal direction, as in the ideal case (Fig. 1). The ERA-40 analyses in this case do not seem able to capture much of the structure in saturation fraction, other than a general moistening along the southern half of the zonally elongated closed gyre at $850 \mathrm{hPa}$ (not shown). The distribution and evolution of TRMM precipitation in this case is similar, nonetheless, to that of Fabio as shown in Fig. B14, with ITCZ convection gradually replaced by convection predominantly in the closed gyre as visualized in the co-moving frame at $600 \mathrm{hPa}$. The excellent correspondence of convection and closed circulation in the co-moving frame (absent in the resting frame) further supports our preference for the co-moving frame in this instance. It is not uncommon for easterly waves and/or ITCZ to extend into the central Pacific from the east, so it is plausible that genesis occurred in a manner similar to the easterly wave cases highlighted above. Whether characterized primarily as "incident waves from upstream" or as "in situ instability of the ITCZ" their westward phase propagation plays a key role in the genesis sequence.

\section{Appendix C}

\section{Topological aspects of the marsupial paradigm}

The evolution to a tropical depression evidently requires formation of closed streamlines in the co-moving frame, comprising a westward-moving cyclonic gyre at meso- $\alpha$. In ERA-40 analyses, closed streamlines are observed prior to TD formation as declared officially, sometimes by $30 \mathrm{~h}$ or more. In the quasi-monochromatic cases examined here, we have identified the tropical wave critical layer with these closed streamlines, known in nonlinear critical layer theory as "Kelvin cat's eyes". One or more cat's eyes aligned zonally are present prior to genesis in virtually all cases. Formation of closed streamlines is also common in quasi-2-D turbulence and involves, as it were, the creation of new vortex coordinate system when a hyperbolic critical point appears in the flow. It is at this point that the Jacobian of the mapping from isentropic PV (or isobaric vorticity) to physical coordinates becomes singular as material contours connecting the vortex to its parent flow are distended into a thin, wispy filament. For practical purposes (as accomplished, e.g., by contour surgery) we end up with a detached vortex with its own identity and closed isopleths of PV (vorticity). In the co-moving frame the good agreement between vorticity isopleths and streamlines or stream function contours, and the near-coincidence of gyre center with eventual TC development in most cases, confirms our choice of translation speed or "gauge" and strongly suggests that the flow kinematics within the critical layer influence mesoscale developments subsequent to gyre formation. Our first hypothesis (H1) is confirmed by these observations.

In a nonlinear critical layer the Lagrangian mean zonal flow is asymptotically discontinuous at the edge of the cat's eyes (Fig. 1; Andrews and McIntyre, 1978) indicating the creation of three separate air masses. In cyclonic latitudinal shear the cat's eyes themselves are cyclonic and their isolation effectively separates air within from the exterior flow. Thus, even though an easterly jet (e.g., AEJ) does not afford the same barrier to meridional transport ${ }^{41}$ as typical of westerly jets in rotating planetary atmospheres (Dunkerton and Scott, 2008) the critical layer south of the jet axis may protect a proto-storm from dry air to the north, air often associated with the Saharan Air Layer (SAL). Such protection is available only if persistent waves exist on the jet with amplitude that is neither too small (as to be insignificant) nor too large (as to draw air from the SAL north of the jet axis) ${ }^{42}$.

\footnotetext{
${ }^{41}$ Among the reasons cited by Dunkerton and Scott for the apparent transport barrier is the fact that a westerly jet core on a stable gradient of PV cannot be the locus of a free Rossby wave critical level/latitude; therefore, isopleths of PV tend to remain elastic at this location (McIntyre, 2003) and irreversible displacements, if any, are limited in meridional extent (e.g., Panetta, 1993). This situation contrasts with an easterly jet core, which can serve as the locus of instability with critical level/latitude near the core (Lindzen et al., 1983) or (if stable) the locus of critical-layer development owing to the weakness of shearing deformation along the jet axis (Brunet and Warn, 1990; Brunet and Haynes, 1995). Whatever barriers to meridional transport that might arise on an easterly jet will depend on the inhomogeneity of stirring owing to the finite extent of the critical layer.

${ }^{42}$ Large-amplitude easterly waves, that is, with large meridional extent, may be ineffective for TC genesis owing to the latter reason. These waves are often observed to precede a new SAL air mass as it sweeps across the tropical Atlantic.
} 


$$
\text { Beryl: SF (2.5-day LP) }
$$
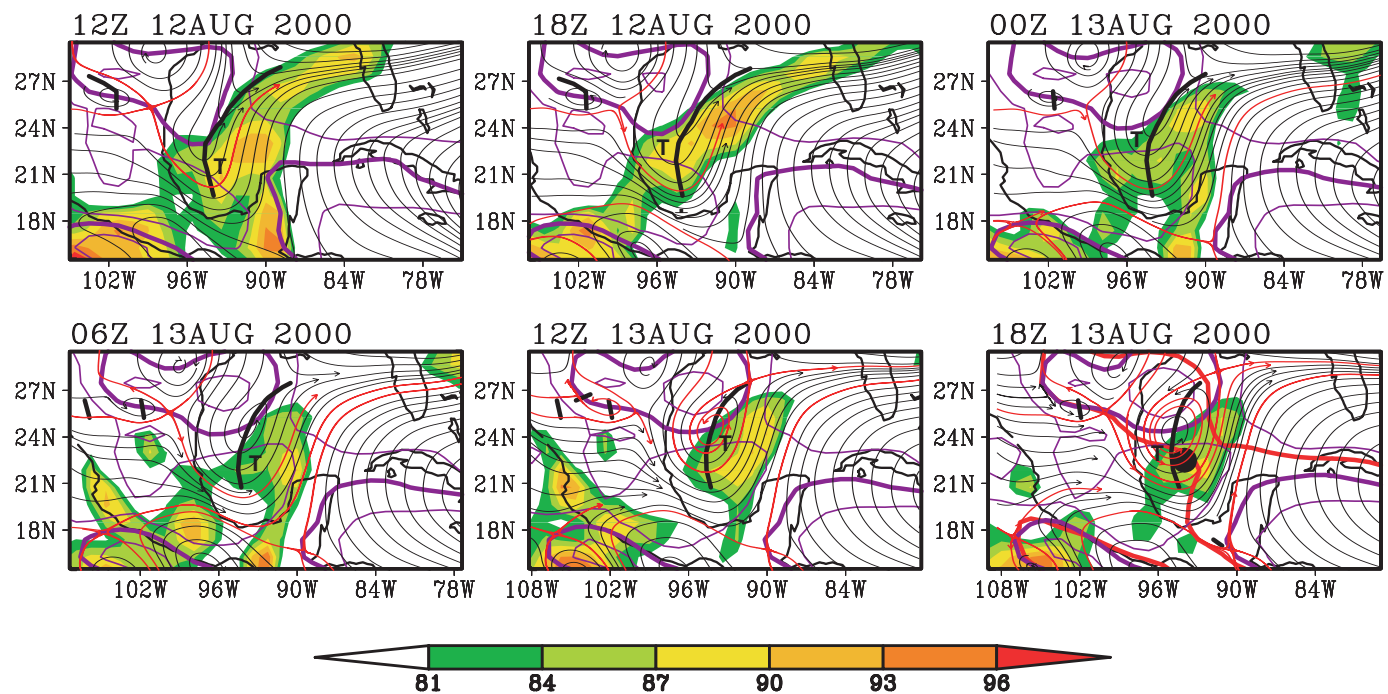

Fig. B9. Streamlines of horizontal flow at $850 \mathrm{hPa}$ for the genesis sequence of Tropical Storm Beryl as in Fig. 28, with high values of ERA-40 saturation fraction indicated by shading (units: percent). The sequence of frames translates westward at the zonal propagation speed of the parent wave at $850 \mathrm{hPa}\left(-3.8 \pm 2.8 \mathrm{~ms}^{-1}\right)$ and streamlines are calculated and displayed in the co-moving frame.

$$
\text { Beryl: TRMM (2.5-day LP) }
$$
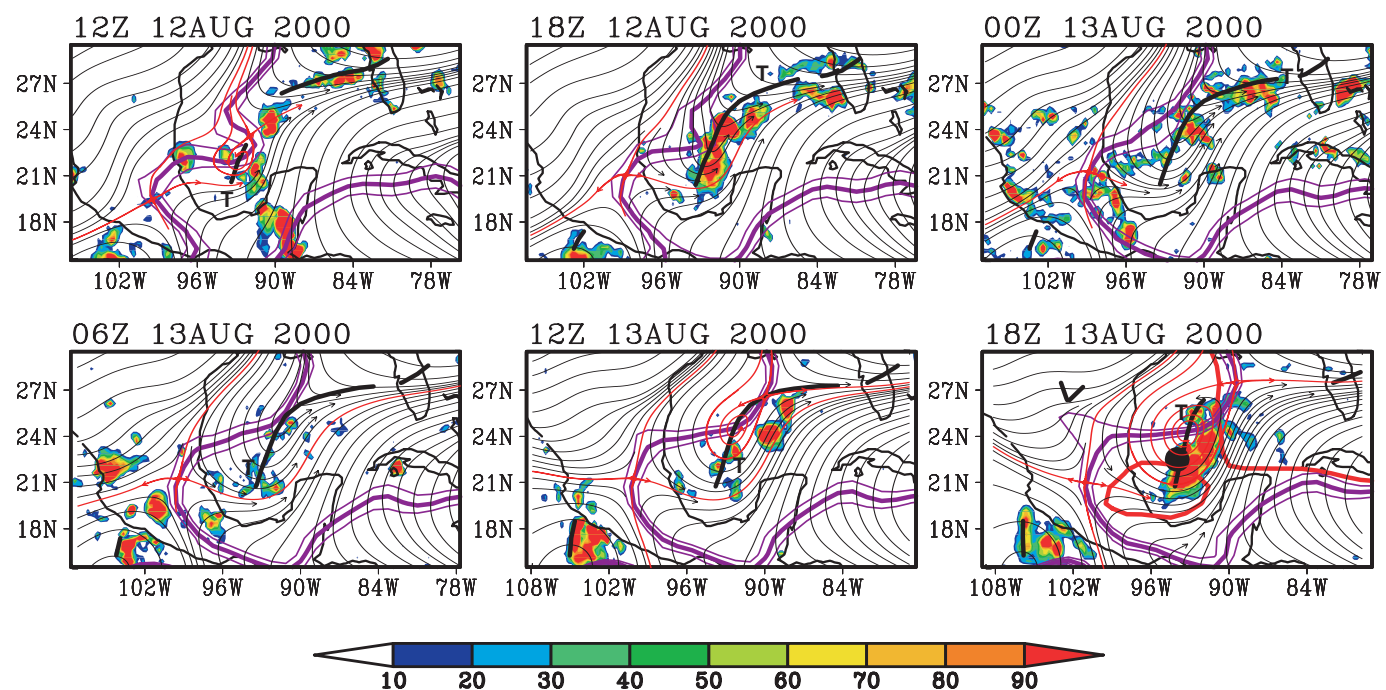

Fig. B10. Streamlines of horizontal flow at $600 \mathrm{hPa}$ for the genesis sequence of Tropical Storm Beryl as in Fig. 29, with high values of TRMM 3-h accumulated precipitation indicated by shading (units: $\mathrm{mm} / \mathrm{day}$ ). The sequence of frames translates westward at the zonal propagation speed of the parent wave at $600 \mathrm{hPa}\left(-3.1 \pm 0.8 \mathrm{~ms}^{-1}\right)$ and streamlines are calculated and displayed in the co-moving frame.

In real life the ideal critical layer sometimes appears, with several Kelvin cat's eyes in the row, but more often takes the form of an isolated vortex surrounded by an asymmetric "teardrop". For both types of streamline configuration, the flow kinematics are very different when viewed in comoving and resting frames. The orientation of separatrix in the co-moving frame, connecting the proto-vortex to the ex- terior flow to the east, north or west, but not south ${ }^{43}$ of the

\footnotetext{
${ }^{43}$ Streamlines of the resting frame are misleading in this respect: an upright omega pattern with separatrix (if any) oriented south of center is usually implied, consistent with Fig. 3c. The similarity of this pattern to the distribution of moisture and low cloud is an unfortunate coincidence, but with careful analysis, a zonal phase lag of $\sim 1 / 4$ wavelength can be detected.
} 


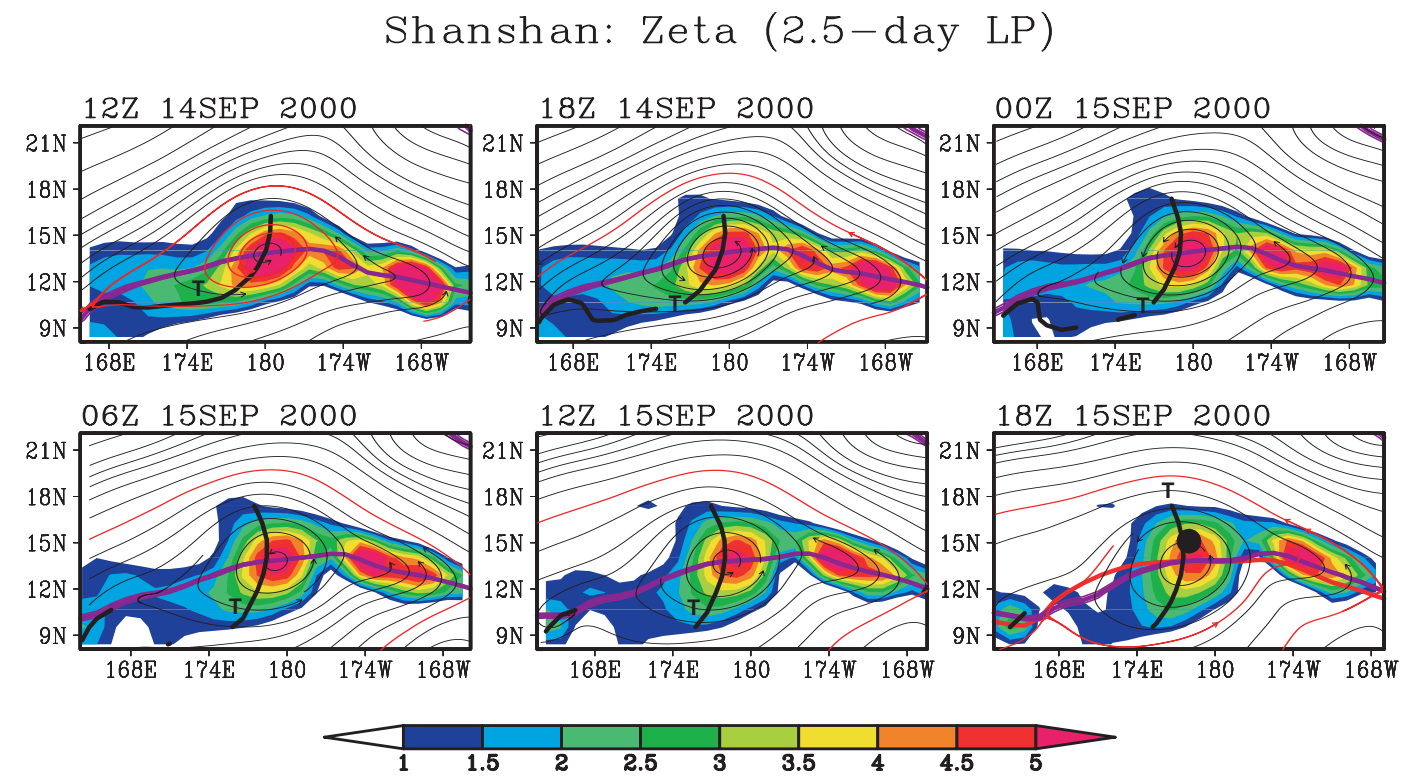

Fig. B11. Streamlines of horizontal (rotational + divergent) flow at $850 \mathrm{hPa}$, as seen in ERA-40 data, for six consecutive analyses leading up to the best-track genesis time of Super Typhoon Shanshan (2000). Shading indicates relative vorticity (units: $10^{-5} \mathrm{~s}^{-1}$ ). The sequence of frames translates westward at the zonal propagation speed of the parent wave at $850 \mathrm{hPa}$ as estimated from the Hovmöller method $\left(-2.8 \pm 0.3 \mathrm{~ms}^{-1}\right)$ and streamlines are calculated and displayed in the co-moving frame; note that relative vorticity itself is invariant with respect to the translation. Isopleths of zero relative zonal flow are shown (purple) together with their uncertainty. In the final panel of the sequence the critical latitude of the parent wave is also indicated (red) corresponding to isopleths of zero relative zonal flow in low-frequency data with periods longer than 9 days. The wave's trough axis is shown for reference (black). The genesis location is indicated by the black dot in the final panel.

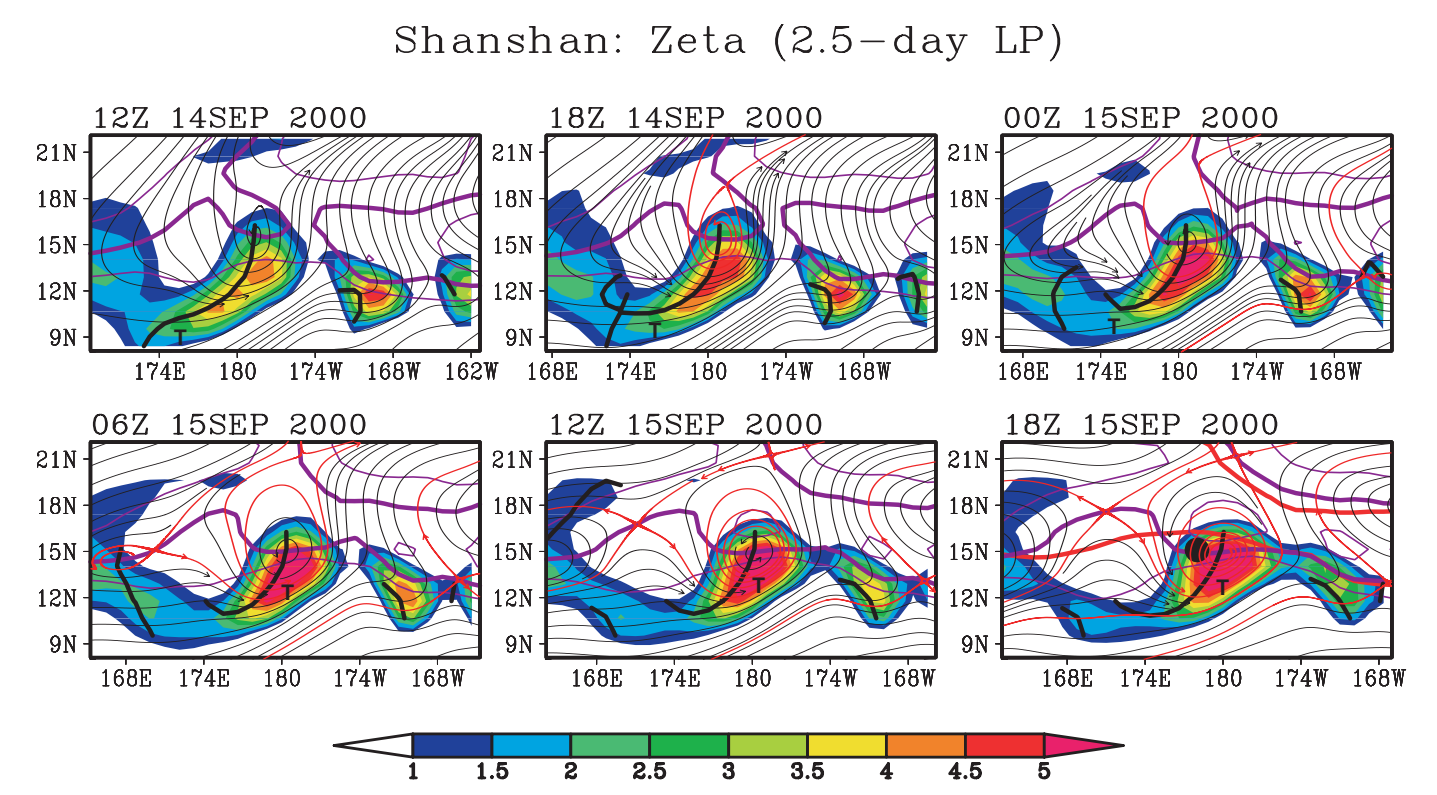

Fig. B12. Streamlines of horizontal flow for the genesis sequence of Super Typhoon Shanshan as in Fig. 33, but at 600 hPa. Shading indicates relative vorticity (units: $10^{-5} \mathrm{~s}^{-1}$ ). The sequence of frames translates westward at the zonal propagation speed of the parent wave at $600 \mathrm{hPa}$ $\left(-5.7 \pm 2.8 \mathrm{~ms}^{-1}\right)$ and streamlines are calculated and displayed in the co-moving frame. 
Shanshan: Zeta (2.5-day LP, resting)

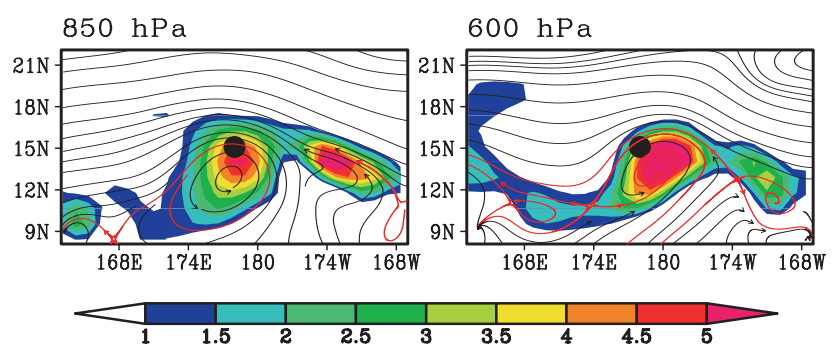

Fig. B13. Streamlines of horizontal flow at (a) $850 \mathrm{hPa}$ and (b) $600 \mathrm{hPa}$ at the genesis time of Super Typhoon Shanshan, in the resting frame. Shading indicates relative vorticity (units: $10^{-5} \mathrm{~s}^{-1}$ ) which is invariant with respect to translation, therefore identical in co-moving and resting frames (cp. final panels of Figs. 33, 34).

gyre center, has two implications:

1. The critical latitude of stable waves lies equatorward of the inflection point where cyclonic vorticity is maximum, therefore the developing gyre is able to tap the relatively high vorticity that exists near the inflection point closer to the axis of the jet ${ }^{44}$.

2. Air entering the gyre, for the most part, is not coming from points far to the south, whether wet (as from a distant ITCZ) or dry (as from the equator). Instead - consistent with our comparison of the analyzed saturation fraction and observed precipitation - moisture is provided to the gyre by moist convection directly. In some cases lateral entrainment of moisture from a nearby ITCZ, slightly south of center, also promotes saturation of the gyre.

Moisture injected into the free troposphere above the boundary layer by deep convection is then transported horizontally, some of it remaining in the gyre, some of it escaping the region of interest. When ambient moisture is also available, it is entrained passively into the cat's eye along with

\footnotetext{
${ }^{44} \mathrm{~A}$ disturbance growing on an unstable meridional gradient of PV would tap mean vorticity at the inflection point equatorward of its critical level. The observed distribution of separatrix location does not preclude instability altogether, but seems to argue against this simplest of instability configurations in the context of TC genesis. A separatrix slightly south of centerline suggests instability originating from a vortex strip to the south, as in ideal simulations of the breakdown of the Northern Hemisphere ITCZ (Wang and Magnusdottir, 2005, 2006). But in most cases there is some zonal variation of the basic state to contend with. A broader definition of "instability" should include (i) eddy shedding to the west of a moist vortex strip (as in the African/Atlantic sector) or (ii) unstable interaction between a monsoon gyre and waves incident from the east (as in the western Pacific). These scenarios, together with our neutral wave scenario, help to account for separatrix orientations to the east, north and west of gyre center.
}

cyclonic vorticity, from adjacent environments (convergence zone or monsoon gyre to the east or west) in which these two variables are positively correlated above the boundary layer. Whether or not entrainment of pre-existing moisture is significant, the Lagrangian boundaries of the developing gyre serve to protect this region from lateral intrusions of dry air. Thus, the same convection that aids the development and concentration of cyclonic vorticity also provides moisture to the column, maintaining the preference for convective over stratiform precipitation - a preference, we have argued above, that favors low-level development. Our second hypothesis (H2) is confirmed, in part, by the juxtaposition of analyzed moisture (ERA-40) and observed deep convective precipitation (TRMM) within the gyre. The morphology of deep convective and stratiform cloud, and inferred profile of diabatic heating in the gyre, also support $\mathrm{H} 2$.

In freely evolving 2-D turbulence or in nonlinear waves on a shear flow, closed recirculation regions occur as a result of horizontal advection induced by the PV itself. Such features are relatively long-lived and robust in their inner core ("coherent structures") while contributing to deformation (or instability) on their flanks and in the neighboring flow. In some sense, coherent structures can be said "to contribute to the forward enstrophy cascade while remaining immune from its immediate effects." In the ideal nonlinear Rossbywave critical layer it is remarkable that the PV responsible for the Kelvin cat's eye is located in the outer, not inner, region; associated, that is, with the large-scale wave and not with the evolving spiral of PV in the cat's eye. This property arises from the morphology of the displacement field near the critical latitude: its local amplification owing to small intrinsic frequency. The same consideration forming the basis of our third hypothesis (H3) is also responsible for the predetermined "wire-frame" structure of streamlines in and around the Kelvin cat's eye. In the context of TC genesis we infer, then, that the details of potential vorticity and PV concentration in the proto-vortex are of secondary importance to the critical layer itself. This organizing principle has a finite lifespan and is eventually superceded in the TC intensification phase when the storm's own PV begins to control the outer environment, leading to the demise of the parent wave and possible excitation of new waves. Our analysis of pre-genesis environments has demonstrated (i) the robustness of the tropical wave critical layer as well as (ii) its contribution to vorticity/moisture entrainment/containment and (iii) protection of the proto-vortex from the exterior flow. These properties suggest a role for the forward enstrophy cascade in TC genesis extending beyond the formation of a tropical wave critical layer in the first place. In the real world, however, simple analogies with waves and 2-D turbulence are inadequate, owing to moist diabatic processes associated with deep convection, an essential ingredient in proto-vortex development. Horizontal advection by synoptic and meso- $\alpha$ motions alone cannot account for TC formation, even though they are expected to create variance at meso- $\beta$ 


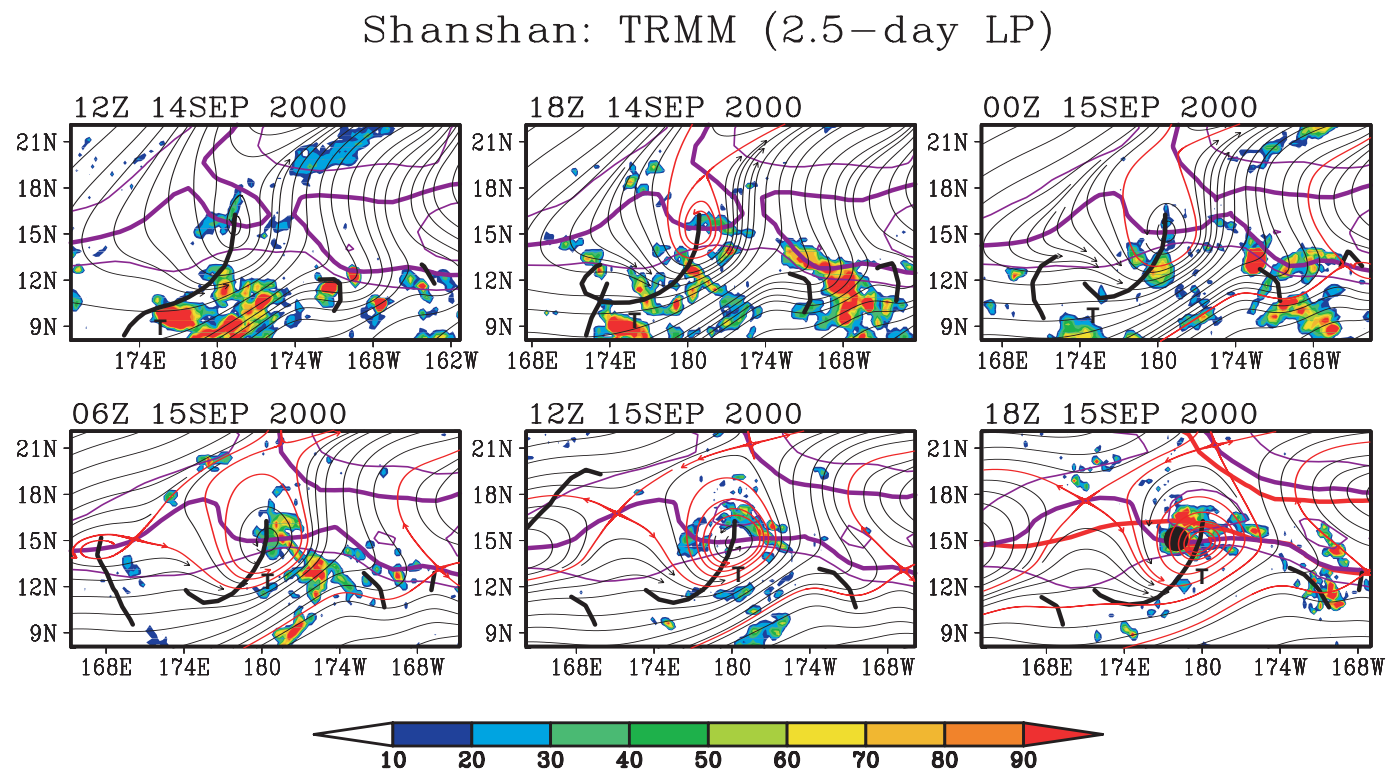

Fig. B14. Streamlines of horizontal flow at $600 \mathrm{hPa}$ for the genesis sequence of Super Typhoon Shanshan as in Fig. 34, with high values of TRMM 3-h accumulated precipitation indicated by shading. Units: $\mathrm{mm} /$ day. The sequence of frames translates westward at the zonal propagation speed of the parent wave at $600 \mathrm{hPa}\left(-5.7 \pm 2.8 \mathrm{~ms}^{-1}\right)$ and streamlines are calculated and displayed in the co-moving frame.

via the forward enstrophy cascade. In discussing vorticity evolution and the topology of streamlines it is essential to distinguish the tropical cyclone from the sub-synoptic gyre in which it forms:

1. In tropical cyclones, isolated vortices are significantly amplified by diabatic processes associated with deep moist convection. It is impossible to obtain the values of vorticity observed in these systems by advective rearrangement alone; cloud system processes enhance vorticity by tilting and stretching of vortex tubes (on the order of 100 times ambient values: M06). This chain of events is itemized in our response to Interactive Comment 4. It is thought by nearly everyone (by topdown and bottom-up schools, in diabatic Ekman turbulence and VHT pathways) that vortices created on the cloud-system scale undergo horizontal upscale aggregation subsequently to form the tropical cyclone; this aggregation further organizes deep convection and vortexscale convergence due to the radial overturning circulation.

2. In the marsupial paradigm, the creation of an isolated vortical air mass by advection in the horizontal plane pertains not to the tropical cyclone itself, but to the gyre in which TC formation may subsequently occur. (The role of upscale aggregation in the critical layer will be discussed in our modeling study.) We believe that whereas upscale aggregation of vorticity and its amplification by deep convection are essential to TC formation, they are not entirely responsible for the formation of a closed tropical depression in the first place. This formation is at least partly due to the dynamics of moist waves and involves a direct enstrophy cascade from larger synoptic-scale disturbances to mesoscale vorticity anomalies. This argument rests on the coherent relationship observed between the critical layers of tropical waves and tropical cyclogenesis events occurring in such regions. Storm formation at or near the center of the translating gyre in many cases seems almost uncanny and suggests to our minds an invisible "guiding hand" from synoptic and meso- $\alpha$ scales.

It should be noted that a predominant role of the Kelvin cat's eye is assigned to the time period leading up to and immediately following genesis. An intensifying storm is distinguished by its increasingly intense concentration of potential vorticity substance, and increasing autonomy as a result. It is a remarkable property of the point-vortex train in parallel shear flow (Pozrikidis, 1997) that a similar cat's eye pattern is produced without any outer disturbance at all. This similarity suggests an almost seamless transition from tropical wave critical layer to (one or more) tropical depressions. We conjecture that the emergence of a developing storm and demise of the parent wave may be identified with such a transition, and quantified by its vorticity concentration relative to that of the original critical layer (as measured, say, by rms values within). This area of research merits further study.

Firmly established in our observations of gyres in the comoving frame is that the advective rearrangement of vorticity draws air with cyclonic vorticity into the cat's eye from an inflection point north of the critical latitude (in 
hydrodynamically stable cases) supporting H1. Such a transport pathway, visible in the co-moving frame but oblivious to observers in the resting frame, does not immediately suggest moistening of the gyre by horizontal advection from a distant ITCZ to the south, nor drying from the equator. The topology of unstable cases differs, with one or more connections slightly south of centerline, suggesting that a developing gyre may entrain moisture from a nearby ITCZ to the south. When this pathway is available, entrained moisture accelerates the approach to column saturation. It was suggested in Sect. 2.2 that chaotic advection associated with mesoscale circulation anomalies may dilute or delay moistening of the cat's eye by deep moist convection. We neglected to say at that point, by way of speculation, that the same anomalies may create low-level vorticity boundaries within the cat's eye that stimulate deep convection via Ekman pumping, providing in situ moistening of the column, an essential ingredient in H2. This is one of a limited number of mesoscale mechanisms that may link chronologically the formation of a closed gyre at meso- $\alpha$ with TC genesis at meso- $\beta$. Another mechanism relies on lateral confinement of small-scale waves excited by convection in the proto-vortex (Ritchie et al., 2003). A third mechanism assists development by lateral confinement of outflow boundaries (M06). All three mechanisms involve some degree of "imbalance" due to boundary-layer drag, inertia-gravity waves or density currents in the lower troposphere. The first mechanism (Ekman triggering) is likely to dominate in a rotating environment approaching column saturation, while the latter mechanisms (involving waves or density currents) typify convective clouds in a sub-saturated environment, with their downdrafts and cold-pool outflow. Outflow boundaries in SAR imagery are distinctly absent in the inner core of developing storms but commonly seen in the outer bands (Katsaros et al., 2000; Black et al., 2005; Dunkerton et al., 2009b). From these precious observations we infer that successful development entails a transition from (i) a canonical MCS mixture of updrafts and downdrafts (with unbalanced motions in all azimuthal directions having a negative net impact on boundary-layer moist entropy) to (ii) a smoother and more axially symmetric in-up-out meridional overturning circulation organized and maintained by a combination of interior heating and Ekman convergence. The radial inflow and resulting tangential circulation assist further development by wind-induced surface evaporation.

A curious observation especially over the eastern Pacific is that the location of TC genesis often lies on or very near a boundary between moist and dry air in the environmental flow. Without further study it is unclear what impact this moisture boundary has either on the dynamics of the incident wave (instability) or the development of coherent structures on the mesoscale. It seems possible that the ambient moisture and its horizontal transport in such situations have a greater role to play than implied in the preceding discussion. A closed gyre with $\sim 50 \%$ overlap of an initially moist region inevitably will entrain the existing moisture in addition to any further moistening by deep convection triggered by the development of the gyre. Such entrainment, as already noted, shortens the time required to saturate the column. This argument does not account, however, for the overlap of gyre and moisture gradient in the first place. Perhaps the moisture gradient is helping to define the effective moist PV gradient (Sobel et al., 2001) seen by the incident wave (instability) and the resulting selection of phase speed, hence, critical latitude. More investigation of this observation is warranted.

An over-arching conclusion emerging from this study regarding easterly waves - whether neutral waves, quasimodes, or hydrodynamic instabilities - is that the juxtaposition of wave critical latitude, inflection point, and moisture gradient (if any) is centrally important to genesis. The first and most easily appreciated conclusion is that the critical latitude south (not north) of the easterly jet axis is the locus of cyclonic vorticity and its subsequent amplification. The second and less obvious conclusion, which becomes obvious in the co-moving frame, is that a neutral or slowlydecaying quasi-mode having its dominant phase speed outside the range of mean flow speeds within the region of reversed potential vorticity gradient is able to tap the mean cyclonic vorticity near the inflection point of the jet. In stable cases (Northern Hemisphere) streamlines in the comoving frame connect northward (via the hyperbolic point) - not southward, as implied in the resting frame. Standard meteorological charts in the Earth-relative frame completely obscure the point. This situation contrasts with that of an unstable disturbance with critical latitude inside this range, closer to the jet axis. In unstable cases, streamlines in the comoving frame connect to the south, although the separatrix orientation may be shallow (ESE or WSW) once the disturbances reach finite amplitude, forming a "curved teardrop" shape poleward of the original location of the ITCZ. A possibly significant role for hydrodynamically stable waves in this context is counter-intuitive: one might be tempted to think that large-scale instability implies a more vigorous sequence of events that would lead to genesis more readily than what might be triggered by a neutral or slowly decaying wave. But we must be willing to entertain less intuitive ideas in order to understand the possible preference for neutral or slowly decaying modes in TC genesis. (i) These modes are maintained by diabatic heating (H3) in addition to their "dry" maintenance (compact spectral content). (ii) Genesis is an interactive multi-scale process and not simply a consequence of moist hydrodynamic instability. If the quasi-mode configuration as described above favors mesoscale development, then it may actually be more favorable for genesis than a more vigorous unstable wave. Oddly enough, the fact that the instability paradigm in reality applies only to a minority of cases seems to support such counter-intuitive thinking. 

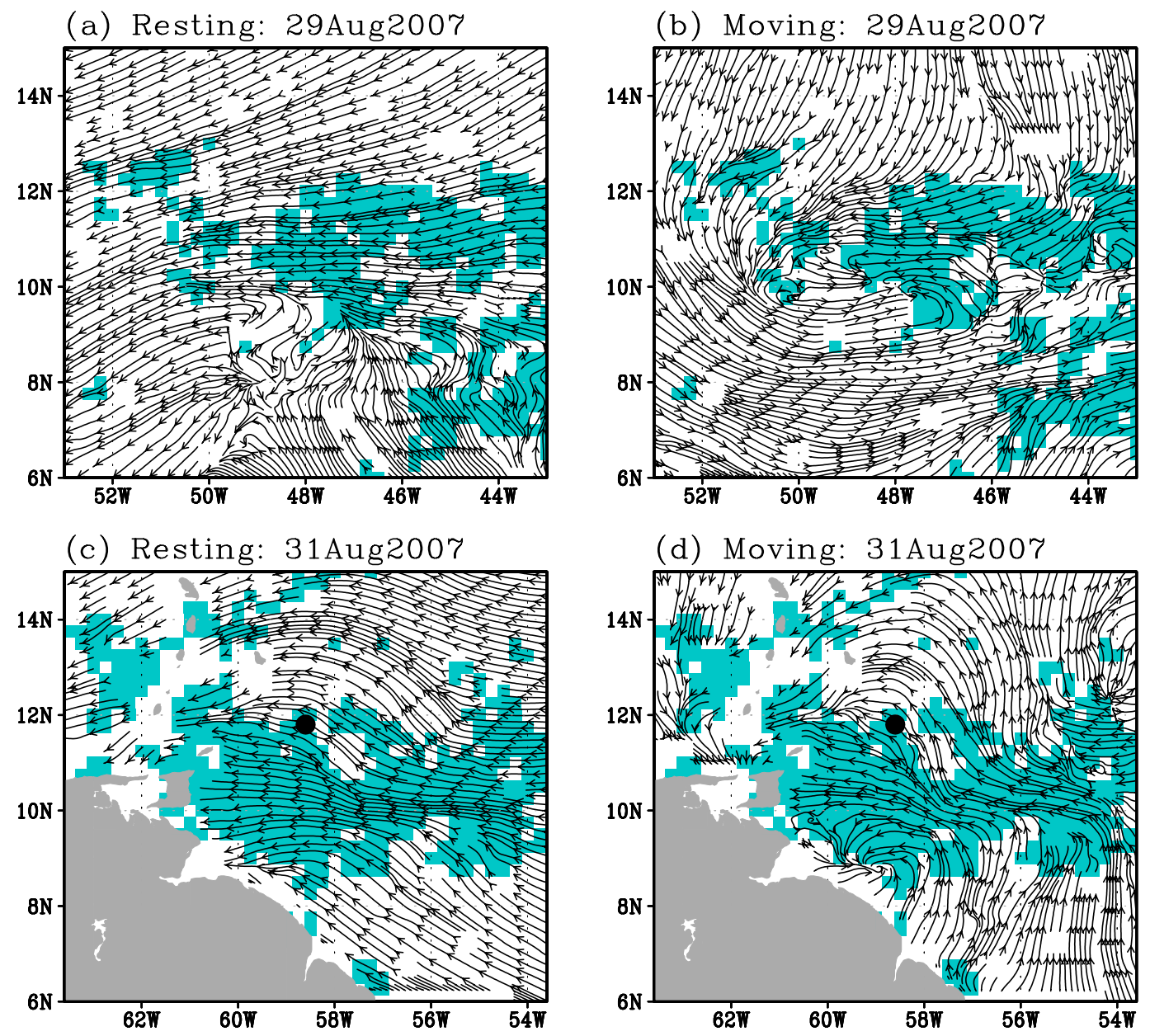

Fig. D1. Streamlines of surface flow obtained from QuikSCAT images of Hurricane Felix (2007) in late August 2007. (a, c) Resting frame; (b, d) co-moving frame $\left(C_{p}=-6.7 \pm 0.5 \mathrm{~ms}^{-1}\right)$. Regions of possible rain contamination are indicated by cyan shading.

\section{Appendix D}

\section{Translation of non-sequential imagery}

Our method for translation of analyses and imagery into a co-moving frame of reference following the parent wave is easily applied to non-sequential images (e.g., sparse or irregular temporal sampling from polar-orbiting satellites) if the wave's propagation speed and direction are known from other data sources. For historical cases a retrospective estimate of $C_{p}$ is straightforward (see text) whereas in realtime applications a one-sided estimate should suffice. In either event, analyses from meteorological centers and global forecast models will provide the requisite information about waves. The existence of a closed gyre is insensitive to small $\left(\sim 1 \mathrm{~ms}^{-1}\right)$ errors in the estimate of $C_{p}$ while its exact location and orientation of flow separatrix are more sensitive to this choice. Larger errors are fatal: as demonstrated in Sect. 3, major differences generally exist between streamline and stream function patterns in a resting frame $\left(C_{p}=0\right)$ and a frame of reference moving westward at a speed typical of Atlantic easterly waves $\left(C_{p} \sim-5\right.$ to $\left.-10 \mathrm{~ms}^{-1}\right)$. We suggest a rule of thumb that a priori information of waves be good enough (sufficiently large and/or persistent waves, plus reliable analysis) to specify $C_{p}$ to within $1-2 \mathrm{~ms}^{-1}$. If this criterion is met, a simple correction to the resting view of non-sequential imagery can be made if the speed of incident waves is reasonably constant. We illustrate the effect of translation on the surface wind field obtained from a QuikSCAT image of Hurricane Felix (2007) in the western Atlantic/eastern Caribbean Sea (Fig. D1). Felix was born within an easterly wave with phase speed typical of central Atlantic waves $\left(C_{p}=-6.7 \pm 0.5 \mathrm{~ms}^{-1}\right)$. By the time of besttrack genesis on 31 August (panels c, d) there was considerable rain contamination of retrieved winds (cyan shading). Similar to what was observed in Chris 2000 , a closed gyre is seen in the co-moving frame (b) $48 \mathrm{~h}$ prior to "genesis" whereas the resting frame (a) on 29 August gives the appearance of an open wave. Once again the resting frame is misleading and misses a key element in the genesis sequence: 
the closure of near-surface circulation. Although our analysis of Felix is retrospective, no temporal filter was needed either in Fig. D1 or in the derivation of phase speed $C_{p}$ on which the zonal translation is based. Rain contamination was not an issue at this time, except in the NE quadrant of the gyre. QuikSCAT imagery evidently can play an important role in identifying a closed surface circulation in the comoving frame, particularly in the earliest stages of genesis when deep convective precipitation is less prevalent or intense than in later stages. It was noted previously by Sharp et al. (2002) that vorticity anomalies in QuikSCAT data can identify storms prior to their best-track genesis time. Vorticity is Galilean invariant but a noisy quantity; translating streamlines provide an alternative measure that is less subject to retrieval errors, unresolved finestructure and rain contamination, provided that the speed of translation is known from other data sources.

\section{Appendix E}

\section{Implications of climate change}

The role of off-equatorial circulations as a link between hurricanes and climate change is recognized in the papers cited. These topics, along with air quality, are the most important in contemporary atmospheric science and their relationship deserves scrutiny at a dynamical, not merely statistical, level of understanding. The effect of climate change on hurricanes is not simply to increase the potential intensity (PI) of a hurricane and thereby increase hurricane intensity ipso facto. Leaving aside frontier matters concerning the formulation and accuracy of current PI theory (Emanuel, 2005; Montgomery et al., 2006a; Smith et al., 2008) actual hurricane intensities form a continuous probability distribution function (PDF) bounded above by a limiting intensity. SST is but one of several factors explaining the PDF and its variation (DeMaria et al., 2005) so it is not merely a question of how PI varies with SST. Nor can we ignore the number of storms reaching tropical depression status and how this might change in the future; the percentage of developers versus wave-troughs is small in the present climate - nowhere near saturation. Though little attention has been given to the issue, perhaps for lack of evidence, the number of storms generated is an integral part of the climate-change question. We consider it unlikely that nature will somehow produce more storms of high intensity without producing more storms at all levels of intensity. A counterpoint was advanced by Held (2007) based on results obtained with a simple GCM: Held and collaborators suggested that while the maximum intensity would increase, the number of intense storms would $d e$ crease, as if to suggest a bound on cumulative storm energy, perhaps owing to available moisture (see also Benggston et al., 2007; Knutson, 2007; Knutson et al., 2007, 2008). However, at a recent workshop Bender (2008) presented new re- sults for the Atlantic basin, using higher resolution to obtain more accurate intensity estimates, indicating that both the intensity and number of intense storms would increase significantly in a "warmer world" scenario. Here, the wings of the intensity distribution are elevated by borrowing from the center of the distribution. (Number conservation is constrained by the experimental design, not predicted.) Owing to the nonlinear relation between the cube of intensity and cumulative storm energy (Emanuel, 2005) such a change cannot occur if available moisture is a limiting factor on intensity. Interestingly, the increase in intense storms was confined primarily to the main development region of the Atlantic basin. Although some theoreticians have expressed acceptance of the "moisture supply" theory, the newer results of Bender and collaborators are consistent with our reasoning. Two reasons underlying this "common sense" viewpoint are (i) it is likely for physical and statistical reasons that if more storms are generated, the number of intense storms will increase "more eggs" once fertilized "imply more chicks, implying more chickens" given enough food - and (ii) regions of genesis and highest intensity are (for the most part) well separated spatially, thanks to the zonal motion of storms, restricting the ability of intense storms to inhibit new storms in their wake to distances of less than $1000 \mathrm{~km}$ (Schumacher et al., 2009). In this respect, storms of the real world differ from those of a horizontally periodic geometry, in which the inhibition can be significant.

\section{Glossary}

bottom-up development: A pathway to tropical cyclogenesis that assigns logical and chronological precedence to phenomena in the lower troposphere prior to the upward growth of a tropical depression-strength vortex. These phenomena may occur at meso- $\alpha$ (e.g., critical layer formation in an easterly wave), meso- $\beta$ (e.g., low-level convergence due to a convective heating profile, Ekman convergence induced by boundary-layer drag) or meso- $\gamma$ (convective heating profile associated with one or more vortical hot towers).

coherent structure: In geophysical (quasi-2-D) turbulence, an unusually large or long-lived flow feature (e.g., solitary wave, isolated horizontal eddy, or train of Kelvin cat's eyes in a nonlinear critical layer) that disrupts locally or episodically the turbulent aspects of the flow: viz., disorder, particle dispersion, and energy or enstrophy cascades. The Great Red Spot on Jupiter is a well-known example.

co-moving frame: A frame of reference translating horizontally at the velocity of the parent wave or proto-vortex. Streamlines or stream function appear different in resting versus co-moving frames, unless the translation speed is zero trivially. The proper choice of translation speed may vary with height owing to vertical shear and wave-vortex interaction.

critical layer: The region surrounding a nonlinear wave's critical latitude or level in shear flow. In the enclosed Kelvin 
cat's eye, particles are trapped and recirculate, rather than being swept one way of the other by the surrounding shear. Reversible undulations of particles immediately adjacent to the cat's eye on either side are included in the definition.

critical points: In 2-D flow, the points where both components of horizontal velocity vector vanish. The nature of a critical point is determined by the local velocity gradient tensor. Two examples are (i) a gyre center, to or from which particles may converge or diverge; (ii) a saddle, or hyperbolic point, where a closed gyre is connected to the outer flow, and particles simultaneously converge (along one axis) and diverge (along the orthogonal axis).

diabatic activation: A term introduced in this paper to describe how a propagating Rossby-like wave (e.g., easterly wave) is maintained or amplified by a diabatic Rossby vortex within. Without diabatic activation, such a wave exists as a dynamical feature in the lower troposphere, whose signature may be seen in low cloud or deep-layer water vapor, but with deep moist convection that is either absent or poorly organized.

diabatic Rossby wave/vortex: A hybrid structure consisting of a low-level vortex, excited and maintained by moist diabatic heating in the lower troposphere, co-existing with a quasi-balanced wave in this layer, such that the wave and vortex interact in a mutually beneficial way Their speeds of horizontal propagation, though not necessarily identical, are similar enough over the lifespan of the disturbance so that the vortex remains in approximately the same phase of the wave.

dividing streamline: In steady 2-D flow, the streamline intersecting a nearby saddle point. Particles on opposite sides of the dividing streamline belong to different manifolds of the flow.

easterly wave: A prominent synoptic-scale feature of the summer tropics, centered ten or more degrees off the equator just poleward of the ITCZ, originating and propagating westward across tropical Africa, the tropical Atlantic, and eastern or central tropical Pacific. They are usually accompanied by cloudiness near the trough, and have been documented in radiosonde data and satellite imagery since the early 1950s and 1970s, respectively.

forward enstrophy cascade: A process in quasi-2-D turbulence whereby strong vortical eddies irreversibly deform weaker eddies into filaments on progressively smaller scales.

gauge function: As used here, a scalar function with zero horizontal Laplacian that can be added to stream function or velocity potential, without altering the vertical component of vorticity, or horizontal divergence. Gauge theories and transformations are widely used in classical and quantum electrodynamics, but have a more general mathematical meaning.

heating profile: In this context, the vertical profile of moist diabatic heating. Heating profiles in the tropics are defined and labeled according to their association with typical precipitating cloud structures, such as deep convective, congestus, stratiform, anvil and shallow cumulus cloud. hydrodynamic instability of ITCZ: In theoretical literature the Intertropical Convergence Zone (ITCZ) is idealized as an east-west vortex strip which, once perturbed, may undergo subsequently a (dry or moist) form of barotropic instability, resulting in excitation of zonally propagating waves and coherent vortices of finite horizontal size embedded therein. According to the marsupial paradigm, a quasi-closed gyre generated by large-scale instability may become the "mother pouch" of an incipient tropical cyclone if environment and mesoscale processes allow.

inverse energy cascade: A process in quasi-2-D turbulence whereby small vortical features merge to form progressively larger features, such as large eddies, Rossby waves and zonal jets. For such a cascade to be maintained, energy input at small scales is necessary.

marsupial paradigm: A conceptual framework introduced in this paper to describe how a hybrid diabatic Rossby wave/vortex (existing in a wave critical layer or in isolation) may become a tropical depression and thereafter, a tropical cyclone. The marsupial paradigm embraces three new hypotheses regarding $(\mathrm{H} 1)$ the vortical organization of the critical layer at meso- $\alpha,(\mathrm{H} 2)$ moisture supply to the proto-vortex and protection from dry or dusty air outside, and (H3) a mutually beneficial interaction of wave and vortex. The simpler term pouch theory has been introduced to encompass $\mathrm{H} 2$.

mesoscale convective vortex: A byproduct of deep moist convection observed in the tropics and summer midlatitudes when an organized mesoscale convective system (MCS) excites horizontal vortical motion in the interior troposphere. The altitude where such motion maximizes depends on the vertical profile of moist diabatic heating. Convection always develops upward, but when accompanied by stratiform cloud at mid-levels (with evaporative cooling below) the resulting profile of moist diabatic heating becomes top-heavy, exciting vortical motion in the mid-troposphere. In tropical cyclogenesis, merger of adjacent MCVs is thought to create a starting point for top-down development.

multi-scale interaction: A mathematical and theoretical concept describing how processes on different scales interact simultaneously, and in some cases, synergistically to support one another. An important result of multi-scale interaction is that turbulent cascades are interrupted or "shortcircuited" by instantaneous communication across widely separated scales. Another is long-range interaction, whereby waves communicate information to large distances, faster than is possible in advective turbulence. The co-existence and possible synergy of waves and turbulence (or of waves and vortices) is arguably the most important form of multiscale interaction in geophysical fluid dynamics.

Okubo-Weiss parameter: A measure of the shapepreserving component of a vortical flow, in comparison to (i) the shape-destroying component associated with shearing deformation, and (ii) straining deformation. The sign convention is arbitrary: "positive" may be chosen to emphasize the former, in shape-preserving phenomena (e.g., TC 
genesis) or the latter, in filamentary phenomena (e.g., stirring and mixing).

organized deep moist convection: Tropical convection, particularly over the oceans, is often organized on subseasonal time scales by atmospheric dynamics rather than by fixed features such as coasts and topography. The form of organization varies widely, and may be associated with mesoscale convective systems, density currents, gravity waves, equatorial waves, and extratropical intrusions. When convection is phase-locked to the underlying dynamic structure to such an extent that this structure cannot be understood without the effect(s) of the convection, we have a convectively coupled disturbance.

Orr mechanism: In shear flow, a wave tilted initially against the shear is rotated to an upright alignment, leading to wave growth, and further rotated into the shear, causing wave decay. The growth rate is determined by the shear, which in some cases may exceed the growth rate of the most unstable eigenmode.

proto-vortex: The initial vortical structure within a hybrid diabatic Rossby wave/vortex, which may subsequently grow to a tropical depression-strength vortex. The finestructure of a proto-vortex is seldom observed directly; even the best meteorological analyses visualize such a disturbance as if "under a pane of knobbly glass" in the famous words of Michael McIntyre.

quasi-mode: Not an exact eigenmode, but a long-lived superposition of modes having approximately the same phase speed; hence, approximately the same critical latitude or level.

saturation fraction: A column-integrated measure of moisture relative to what the column can hold, given the observed temperature versus pressure in the column.

spontaneous aggregation: In idealized studies of tropical cyclogenesis or diabatic Ekman turbulence in horizontally uniform environments without waves or sweet spots, a mechanism whereby adjacent vortical elements merge adiabatically via the inverse energy cascade, or diabatically via nonadvective transport of PV substance, and grow subsequently to form a tropical cyclone.

stratified turbulence paradigm: Based on the pioneering works of Jim Riley, Doug Lilly and Jim McWilliams (in the context of geophysical turbulence) and as applied to tropical meteorology (invoking the scale analysis of Jule Charney and Jim Holton), a concept that describes the seemingly random relationship of lower and upper tropospheric flows when deep coupling is absent. For example, when an extratropical disturbance intrudes the tropical upper troposphere, without altering a pre-existing disturbance in the tropical lower troposphere, a vertical shear may be generated that is either favorable or inimical to tropical cyclogenesis; the point being, the superposition is a result of separate processes having no immediate relationship to one another.

top-down development: A pathway to tropical cyclogenesis that assigns logical and chronological precedence to phe- nomena in the middle troposphere prior to their downward extension to the lower troposphere or boundary layer. As with bottom-up development, these phenomena may occur at meso- $\alpha$ (e.g., downward gyre development in an easterly wave, thermal capping in the cat's eye) or meso- $\beta$ (e.g., descending showerhead, downward burrowing of merged midlevel convective vortices). Upward and downward pathways are not mutually exclusive, insofar as the meso- $\alpha$ development may occur in one direction, while the meso- $\beta$ development occurs in another.

translating closed gyre: A structure that may be identified in meteorological analyses of stream function, streamlines or trajectories, by viewing a tropical wave critical layer in its co-moving frame, i.e., a frame of reference moving at the phase speed of the wave. For stream function, the translation can be achieved directly by adding the appropriate gauge function.

tropical depression: Although no formal definition can be found, a tropical depression-strength vortex is thought to exist when circular horizontal winds approach 30 knots and deep, persistent convection is well-organized by a putative closed circulation. It can be said that forecasters regard a proto-structure with features loosely resembling a mature hurricane as a good candidate for a "depression" - the more such features, the better. Their classification evidently guarantees (in all but a few instances) subsequent growth to a named tropical storm. It is not only the statistical narrowness of the tropical depression classification, but a physical threshold for instability leading to intensification, that is responsible for their success. In other words, "tropical depression" is a physically meaningful, albeit imprecisely defined, concept.

vortical hot towers: Deep moist convective clouds that rotate as an entity and/or contain updrafts that rotate in helical fashion (as in rotating Rayleigh-Benard convection). Although early observations suggested that VHTs are neither necessary nor sufficient for tropical cyclogenesis, it is becoming increasingly clear from cloud-representing numerical simulations that moist vortical updrafts are the essential building blocks of the tropical storm within the rotating proto-vortex. These hot vortical plume structures amplify pre-existing cyclonic vorticity and equivalently induce concentrations of vorticity substance much larger than that of the aggregate vortex.

wave refraction: When a wave propagates in a background state with spatio-temporal variation, its wavenumber and frequency change owing to refraction. In shear flow, wave phase may be imagined to open or close like a Venetian blind; this process occurs in the two spatial dimensions orthogonal to the wave vector. Phases may be stretched or compressed, as a piece of taffy; this process occurs in the spatial dimension parallel to the wave vector. The Orr mechanism arises from the first effect, whereas "wave accumulation" or "wave capture" arise from the second. A local increase of wave amplitude at first order caused by linear wave 
refraction may result in wave breaking, or transfer of energy to other waves, vortices and turbulence.

Acknowledgements. Our work was motivated and guided, in part, by, by the continuous and tireless efforts of operational forecasters at the National Hurricane Center/Tropical Prediction Center of NOAA, whose skill at teasing rotational signals out of remotely sensed imagery is unsurpassed. Helpful reviews of the Discussion Paper were provided by David Raymond, Gilbert Brunet, Michael McIntyre and Volkmar Wirth. We thank Michael Riemer for an important clarification. This research was supported by the National Aeronautics and Space Administration, MIPR NNG07HU171 and Contract NNH04CC63C, the National Science Foundation Grants ATM-0715426, ATM-0649944, ATM-0649946, ATM-0227632 and ATM-0733380, the Office of Naval Research grant N001408WR20129 and by the US Naval Postgraduate School in Monterey, California.

Edited by: P. Haynes

\section{References}

Aiyyer, A. R. and Molinari, J.: Evolution of mixed Rossby-gravity waves in idealized MJO environments, J. Atmos. Sci., 60, 28372855, 2003.

Aiyyer, A. and Molinari, J.: MJO and tropical cyclogenesis in the Gulf of Mexico and Eastern Pacific: case study and idealized numerical modeling, J. Atmos. Sci., 65, 2691-2704, 2008.

Alexander, M. J. and Dunkerton, T. J.: A spectral parameterization of mean-flow forcing due to breaking gravity waves, J. Atmos. Sci., 56, 4167-4182, 1999.

Andrews, D. G. and McIntyre, M. E.: An exact theory of nonlinear waves on a Lagrangian mean flow, J. Fluid Mech., 89, 609-646, 1978.

Andrews, D. G., Holton, J. R., and Leovy, C. B.: Middle Atmosphere Dynamics, Academic Press, 504 pp, 1987.

Bassom, A. P. and Gilbert, A. D.: The spiral wind-up and dissipation of vorticity and a passive scalar in a strained planar vortex, J. Fluid Mech., 398, 245-270, 1999.

Bender, M.: Simulation of intense Atlantic hurricane activity in a twenty-first century warmed climate, using the GFDL highresolution, coupled hurricane model, 3rd Workshop on Highresolution and Cloud Modeling Tropical Cyclones and Climate, University of Hawaii at Manoa, 2008.

Bengtsson, L., Hodges, K. I., Esch, M., Keenlyside, N., Kornblueh, L., Luo, J.-J., and Yamagata, T.: How may tropical cyclones change in a warmer climate?, Tellus, 59A, 539-561, 2007.

Berry, G. J. and Thorncroft, C.: Case study of an intense African easterly wave, Mon. Weather Rev., 133, 752-766, 2005.

Bessafi, M. and Wheeler, M. C.: Modulation of South Indian Ocean tropical cyclones by the Madden-Julian oscillation and convectively coupled equatorial waves, Mon. Weather Rev., 134, 638656, 2006.

Bister, M. and Emanuel, K. A.: The genesis of Hurricane Guillermo: TEXMEX analyses and a modeling study, Mon. Weather Rev., 125, 2662-2682, 1997.

Black, P. G., Katsaros, K. B., Drennan, W. M., Lehner, S., and Vachon, P. W.: Interpretation of SAR-observed boundary layer flow structures in hurricanes. NASA Final Report, Work Order No. W-19-835, 58 pp, 2005.

Borth, H.: Fundamental circulation modes in moist rotating atmospheres. Talk presented at a workshop on tropical dynamics, University of Mainz, Mainz DE, 2007.

Bosart, L.: The tropical transition of hurricane Alex (2004): An observational perspective, 27th Conference on Hurricanes and Tropical Meteorology, American Meteorological Society, Monterey CA, 2006.

Bracken, W. E. and Bosart, L. F.: The role of synoptic-scale flow during tropical cyclogenesis over the North Atlantic Ocean, Mon. Weather Rev., 128, 353-376, 2000.

Bretherton, C. S., Peters, M. E., and Back, L. E.: Relationships between water vapor path and precipitation over the tropical oceans, J. Climate, 17, 1517-1528, 2004.

Brill, O. L. and Goodman, B.: Causality in the Coulomb gauge, Am. J. Phys., 35, 832-837, 1967.

Brunet, G. and Warn, T.: Rossby wave critical layers on a jet, J. Atmos. Sci., 47, 1173-1178, 1990.

Brunet, G. and Haynes, P.: The nonlinear evolution of disturbances to a parabolic jet, J. Atmos. Sci., 52, 464-477, 1995.

Burpee, B.: Characteristics of North African easterly waves during the summers of 1968 and 1969, J. Atmos. Sci., 31, 1556-1570, 1974.

Carlson, T. N.: Synoptic histories of three African disturbances that developed into Atlantic hurricanes, Mon. Weather Rev., 97, 256276, 1969.

Chang, C. P., Morris, V. F., and Wallace, J. M.: A statistical study of easterly waves in the western Pacific: July-December 1964, J. Atmos. Sci., 27, 195-201, 1970.

Chang C.-P., Liu, C.-H., and Kuo, H.-C.: Typhoon Vamei: an equatorial tropical cyclone formation, Geophys. Res. Lett., 30(3), 5053, 2003.

Crum, F. X. and Dunkerton, T. J.: Analytic and numerical models of wave-CISK with conditional heating, J. Atmos. Sci., 49, 16931708, 1992.

Crum, F. X. and Dunkerton, T. J.: CISK and evaporation-wind feedback with conditional heating on an equatorial beta-plane, J. Meteorol. Soc. Japan, 72(1), 11-18, 1994.

Davis, C. A. and Bosart, L. F.: Numerical simulations of the genesis of Hurricane Diana (1984). Part I: control simulation, Mon. Weather Rev., 129, 1859-1881, 2001.

Davis, C. A. and Bosart, L. F.: The TT problem: forecasting the tropical transition of cyclones, B. Am. Meteorol. Soc., 85, 16571662, 2004.

DeMaria, M., Mainelli, M., Shay, L. K., Knaff, J. A., and Kaplan, J.: Further improvements to the Statistical Hurricane Intensity Prediction Scheme (SHIPS), Wea. Forecasting, 20, 531-543, 2005.

Dickinson, M. and Molinari, J.: Mixed Rossby-gravity waves and western Pacific tropical cyclogenesis. Part I: synoptic evolution, J. Atmos. Sci., 59, 2183-2196, 2002.

Doblas-Reyes, F. J. and Déqué, M.: A flexible bandpass filter design procedure applied to midlatitude intraseasonal variability, Mon. Weather Rev., 126, 3326-3335, 1998.

Dunion, J. P. and Velden, C. S.: The impact of the Saharan Air Layer on Atlantic tropical cyclone activity, B. Am. Meteorol. Soc., 85, 353-365, 2004.

Dunkerton, T. J.: Intensity variation and coherence of 3-6 day equatorial waves, Geophys. Res. Lett., 18(8), 1469-1472, 1991. 
Dunkerton, T. J.: Observation of 3-6-day meridional wind oscillations over the tropical Pacific, 1973-1992: vertical structure and interannual variability, J. Atmos. Sci., 50, 3292-3307, 1993.

Dunkerton, T. J.: Evidence of meridional motion in the summer lower stratosphere adjacent to monsoon regions, J. Geophys. Res., 100(D8), 16675-16688, 1995.

Dunkerton, T. J.: A tale of two ITCZs - the Jim Holton perspective, B. Am. Meteorol. Soc., 87, 1492-1495, 2006.

Dunkerton, T. J. and Baldwin, M. P.: Observation of 3-6-day meridional wind oscillations over the tropical Pacific, 1973-1992: horizontal structure and propagation, J. Atmos. Sci., 52, 1585-1601, 1995.

Dunkerton, T. J. and Crum, F. X.: Eastward propagating 2- to 15day equatorial convection and its relation to the tropical intraseasonal oscillation, J. Geophys. Res., 100(D12), 25781-25790, 1995.

Dunkerton, T. J. and Scott, R. K.: A barotropic model of the angular-momentum conserving potential vorticity staircase in spherical geometry, J. Atmos. Sci., 65, 1105-1136, 2008.

Dunkerton, T. J., Lussier III, L. L., Montgomery, M. T., Wang, Z., and Tory, K. J.: Spatial and statistical distribution of convective and stratiform clouds in the gyre-pouch of incipient tropical cyclones, J. Geophys Res., in preparation, 2009a.

Dunkerton, T. J., Walter, B. A., Perrie, W., Long, D. G., Nie, C., Zhang, J., Rogers, R., Uhlhorn, E., and Black, P.: Images of Hurricane Katrina (2005) below the cloud, Science, in preparation, 2009b.

Edson, R. T. and Lander, M.: Characteristics of the early stages of tropical cyclones as viewed with microwave data, AMS 27th Conference on Hurricanes and Tropical Meteorology, Monterey CA, 2006.

Emanuel, K. A.: The finite-amplitude nature of tropical cyclogenesis, J. Atmos. Sci., 46, 3431-3456, 1989.

Emanuel, K. A.: The physics of tropical cyclogenesis over the eastern Pacific, in: Tropical Cyclone Disasters: Proceedings of ICSU/WMO International Symposium, edited by: Lighthill, J. Zhemin, Z., Holland, G. J., and Emanuel, K., Peking University Press, 136-142, 1993.

Emanuel, K. A.: Some aspects of hurricane inner-core dynamics and energetics, J. Atmos. Sci., 54, 1014-1026, 1997.

Emanuel, K. A.: Increasing destructiveness of tropical cyclones over the past 30 years, Nature, 436, 686-688, 2005.

Emanuel, K. A. and Nolan, D. S.: Tropical cyclone activity and the global climate system. Talk presented at the AMS 26th Conference on Hurricanes and Tropical Meteorology, Miami, FL, 2004.

Emanuel, K. A.: Divine Wind: The History and Science of Hurricanes, Oxford University Press, New York, 285 pp, 2005.

Emanuel, K. A.: The hurricane embryo. Talk presented at short program workshop entitled Small scales and extreme events: The Hurricane, NSF Institute for Pure and Applied Mathematics (IPAM), UCLA, 2007.

Eliassen, A.: Slow thermally or frictionally controlled meridional circulation in a circular vortex, Astrophys. Norv., 5, 19-60, 1951.

Eliassen, A.: On the Ekman layer in a circular vortex, J. Meteorol. Soc. Japan, 49, 784-789, 1971.

Eliassen, A. and Lystad, M.: The Ekman layer of a circular vortex: A numerical and theoretical study, Geophys. Norv., 31, 1-16, 1977.

Ferreira, R. N. and Schubert, W. H.: Barotropic aspects of ITCZ breakdown, J. Atmos. Sci., 54, 261-285, 1997.

Frank, N. L.: Atlantic tropical systems of 1969, Mon. Weather Rev., 98, 307-314, 1970.

Frank, N. L. and Johnson, H. M.: Vortical cloud systems over the tropical Atlantic during the 1967 hurricane season, Mon Weather Rev., 97, 124-129, 1969.

Frank, W. M. and Roundy, P. E.: The role of tropical waves in tropical cyclogenesis, Mon. Weather Rev., 134, 2397-2417, 2006.

Frank, W. M. and Young, G. S.: The interannual variability of tropical cyclones, Mon. Weather Rev., 135, 3587-3598, 2007.

Gentry, R. C., Fujita, T. T., and Sheets, R. C.: Aircraft, spacecraft, satellite and radar observations of Hurricane Gladys, 1968, J. Appl. Meteor., 9, 837-850, 1970.

Goldenberg, S. B. and Shapiro, L. J.: Physical mechanisms for the association of El Niño and West African rainfall with Atlantic major hurricane activity, J. Climate, 9, 1169-1187, 1996.

Gore, A.: An Inconvenient Truth, Paramount Classics, US gross revenue to date: $\$ 49,047,567,2006$.

Gray, W. M.: Atlantic seasonal hurricane frequency. Part I: El Niño and $30 \mathrm{mb}$ Quasi-Biennial Oscillation influences, Mon. Weather Rev., 112, 1649-1668, 1984a.

Gray, W. M.: Atlantic seasonal hurricane frequency. Part II: forecasting its variability, Mon. Weather Rev., 112, 1669-1683, 1984b.

Gray, W. M.: The formation of tropical cyclones, Meteorol. Atmos. Phys., 67, 37-69, 1998.

Green, M. A., Rowley, C. W., and Haller, G.: Detection of Lagrangian coherent structures in 3D turbulence, J. Fluid Mech., 572, 111-120, 2007.

Grotjahn, R.: Different data, different general circulations? A comparison of selected fields in NCEP/DOE AMIP-II and ECMWF ERA-40 Reanalyses, Dyn. Atmos. Oceans, 44, 108-142, 2008.

Hack, J. J. and Schubert, W. H.: Nonlinear response of atmospheric vortices to heating by organized cumulus convection, J. Atmos. Sci., 43, 1559-1573, 1986.

Hack, J. J., Schubert, W. H., Stevens, D. E., and Kuo, H.-C.: Response of the Hadley circulation to convective forcing in the ITCZ, J. Atmos. Sci., 46, 2957-2973, 1989.

Hall, N. M. J., Kiladis, G. N., and Thorncroft, C. D.: Threedimensional structure and dynamics of African easterly waves. Part II: dynamical modes, J. Atmos. Sci., 63, 2231-2245, 2006.

Haller, G. and Yuan, G.: Lagrangian coherent structures and mixing in two-dimensional turbulence, Physica D, 147, 352-370, 2000.

Halverson, J., Black, M., Braun, S., et al.: NASA's Tropical Cloud Systems and Processes Experiment, B. Am. Meteorol. Soc., 88, 867-882, 2007.

Harr, P. A. and Elsberry, R.: Structure of a mesoscale convective system embedded in typhoon Robyn during TCM-93, Mon. Weather Rev., 124, 634-652, 1996.

Hartmann, D. L., Hendon, H. H., and Houze, R. A.: Some implications of the mesoscale circulations in tropical cloud clusters for large-scale dynamics and climate, J. Atmos. Sci., 41, 113-121, 1984.

Hayashi, Y.: A theory of large-scale equatorial waves generated by condensation heat and accelerating the zonal wind, J. Meteorol. Soc. Japan, 48(2) 140-160, 1970.

Heifetz, E., Bishop, C. H., and Alpert, P.: Counter-propagating Rossby waves in the barotropic Rayleigh model of shear instability, Q. J. Roy. Meteorol. Soc., 125, 2835-2853, 1999. 
Held, I. M.: Rotating radiative-convective equilibria at low resolution in large domains, Talk presented at short program workshop entitled Small scales and extreme events: The Hurricane, NSF Institute for Pure and Applied Mathematics (IPAM), UCLA, 2007.

Held, I. M. and Ting, M.: Orographic versus thermal forcing of stationary waves: the importance of the mean low-level wind, J. Atmos. Sci., 47, 495-500, 1990.

Hendon, H. H. and Liebmann, B.: The structure and annual variation of antisymmetric fluctuations of tropical convection and their association with Rossby-gravity waves, J. Atmos. Sci., 48, 2127-2140, 1991.

Hendricks, E. A., Montgomery, M. T., and Davis, C. A.: On the role of "vortical" hot towers in the formation of tropical cyclone Diana, J. Atmos. Sci., 61, 1209-1232, 2004.

Hendricks, E. A. and Montgomery, M. T.: Rapid scan views of convectively generated mesovortices in sheared tropical cyclone Gustav (2002), Wea. Forecasting, 21, 1041-1050, 2006.

Herring, J. R. and McWilliams, J. C.: Lecture notes on turbulence from the NCAR-GTP summer school, June 1987. Lecture notes by Douglas Lilly, p. 171-218, World Scientific, ISBN 9971-50805-2, 1989.

Hidalgo, J. M.: Vortical hot towers, their aggregate effects and their resolution dependence in the formation of Hurricane Diana (1984), $\mathrm{PhD}$ dissertation, Colorado State University, 184 pp., 2007.

Holton, J. R.: An Introduction to Dynamic Meteorology, Elsevier Academic Press, 4th Ed., 535 pp., 2004.

Hoskins, B. J., McIntyre, M. E., and Robertson, A. W.: On the use and significance of isentropic potential vorticity maps, Q. J. Roy. Meteorol. Soc., 111, 877-946, 1985.

Houze Jr., R. A.: Observed structure of mesoscale convective systems and implications for large-scale heating, Q. J. Roy. Meteorol. Soc., 115, 425-461, 1989.

Ide, K., Kuznetsov, L., and Jones, C. K. R. T.: Lagrangian data assimilation for point-vortex systems, J. Turbulence, 3, 053, 1-7, 2002.

IPCC: Climate Change 2007, Fourth Assessment Report (AR4) of the United Nations Intergovernmental Panel on Climate Change, 2007.

Julian, P. R.: Objective analysis in the tropics: a proposed scheme, Mon. Weather Rev., 112, 1752-1767, 1984.

Katsaros, K. B., Vachon, P. W., Black, P. G., Dodge, P. P., and Uhlhorn, E. W.: Wind fields from SAR: could they improve our understanding of storm dynamics?, Johns Hopkins APL Tech. Digest, 21(1), 86-93, 2000.

Kiladis, G. N., Thorncroft, C. D., and Hall, N. M. J.: Threedimensional structure and dynamics of African easterly waves. Part I: observations, J. Atmos. Sci., 63, 2212-2230, 2006.

Killworth, P. D. and McIntyre, M. E.: Do Rossby-wave critical layers absorb, reflect or overreflect?, J. Fluid Mech., 161, 449-492, 1985.

Kimball, S. K. and Mulekar, M. S.: A 15-year climatology of North Atlantic tropical cyclones. Part I: size parameters, J. Climate, 17, 3555-3575, 2004.

Knutson, T. R.: High resolution modeling of hurricanes in a climate context, presentation at AGU Fall Meeting, San Francisco, 2007.

Knutson, T. R., Sirutis, J. J., Garner, S. T., Held, I. M., and Tuleya, R. E.: Simulation of the recent multidecadal increase of Atlantic hurricane activity using an 18-km-grid regional model, B. Am. Meteorol. Soc., 88, 1549-1565, 2007.

Knutson, T. R., Sirutis, J. J., Garner, S. T., Vecchi, G. A., and Held, I. M.: Simulated reduction in Atlantic hurricane frequency under twenty-first-century warming conditions, Nature Geoscience, 1, 359-364, doi:10.1038/ngeo202, 2008.

Krishnamurti, T. N., Bedi, H. S., Oosterhof, D., and Hardiker, V.: The formation of Hurricane Frederic (1979), Mon. Weather Rev., 122, 1050-1074, 1994.

Kurgansky, M. V.: Helicity production and maintenance in a baroclinic atmosphere, Meteorologische Zeitschrift, 15, 409-416, 2006.

Lapeyre, G.: Topologie du mélange dans un fluide turbulent géophysique, Doctoral Thesis, Univ. of Paris, 204 pp., 2000.

Lau, K.-H. and Lau, N.-C.: Observed structure and propagation characteristics of tropical summertime synoptic scale disturbances, Mon. Weather Rev., 118, 1888-1913, 1990.

LeMone, M. A., Zipser, E. J., and Trier, S. B.: The role of environmental shear and thermodynamic conditions in determining the structure and evolution of mesoscale convective systems during TOGA COARE, J. Atmos. Sci., 55, 3493-3518, 1998.

Liebmann, B. and Hendon, H. H.: Synoptic-scale disturbances near the equator, J. Atmos. Sci., 47, 1463-1479, 1990.

Lighthill, M. J.: Waves in Fluids, Cambridge University Press, 504 pp., 1978.

Lilly, D. K.: Stratified turbulence and the mesoscale variability of the atmosphere, J. Atmos. Sci., 40, 749-761, 1983.

Lilly, D. K.: The structure and propagation of rotating convective storms. Part I: energy exchange with the mean flow, J. Atmos. Sci., 43, 113-125, 1986a.

Lilly, D. K.: The structure and propagation of rotating convective storms. Part II: helicity and storm stabilization, J. Atmos. Sci., 43, 126-140, 1986b.

Lin, J., Mapes, B., Zhang, M., and Newman, M.: Stratiform precipitation, vertical heating profiles, and the Madden-Julian Oscillation, J. Atmos. Sci., 61, 296-309, 2004.

Lindzen, R. S.: Wave-CISK in the tropics, J. Atmos. Sci., 31, 156179, 1974.

Lindzen, R. S. and Tung, K. K.: Wave overreflection and shear instability, J. Atmos. Sci., 35, 1626-1632, 1978.

Lindzen, R. S., Farrell, B. F., and Rosenthal, A.: Absolute barotropic instability and monsoon depressions, J. Atmos. Sci., 40, 1178-1184, 1983.

Lukovich, J. V. and Shepherd, T. G.: Stirring and mixing in twodimensional divergent flow, J. Atmos. Sci., 62, 3933-3954, 2005.

Magnusdottir, G.: The modeled response of the mean winter circulation to zonally averaged SST trends, J. Climate, 14, 41664190, 2001.

Maloney, E. D. and Dickinson, M. J.: The intraseasonal oscillation and the energetics of summertime tropical western North Pacific synoptic-scale disturbances, J. Atmos. Sci., 60, 2153-2168, 2003.

Maloney, E. D. and Hartmann, D. L.: Modulation of eastern North Pacific hurricanes by the Madden-Julian oscillation, J. Climate, 13, 1451-1460, 2000.

Mandelbrot, B. B.: Intermittent turbulence in self-similar cascades: divergence of high moments and dimension of the carrier, J. Fluid Mech., 62, 331-358, 1974.

Mapes, B. E. and Houze, R. A.: Diabatic divergence profiles in 
western Pacific mesoscale convective systems, J. Atmos. Sci., 52, 1807-1828, 1995.

Matsuno, T.: Kelvin waves, internal gravity waves and their behaviors in numerical models, Talk presented at the 86th AMS Annual Meeting, Atlanta GA, 2006.

Matsuno, T.: Modeling tropical convective cloud systems by a very high resolution atmosphere model NICAM. Talk presented at ESSL, National Center for Atmospheric Research, Boulder CO, 2007.

McBride, J. L. and Zehr, R.: Observational analysis of tropical cyclone formation. Part II: comparison of non-developing versus developing systems, J. Atmos. Sci., 38, 1132-1151, 1981.

McIntyre, M. E.: On the Antarctic ozone hole, J. Atmos. Terr. Phys., 51, 2973-2994, 1989.

McIntyre, M. E.: Isentropic distributions of potential vorticity and their relevance to tropical cyclone dynamics, Tropical Cyclone Disasters: Proceedings of ISCU/WMO International Symposium, edited by: Lighthill, J. Zhemin, Z., Holland, G. J., and Emanuel, K., Peking University Press, 143-156, 1993.

McIntyre, M. E.: On global-scale atmospheric circulations, in: Perspectives in Fluid Dynamics: A Collective Introduction to Current Research, edited by: Batchelor, G. K., Moffatt, H. K., and Worster, M. G., Cambridge, University Press, paperback edition, 557-624, 2003.

McWilliams, J. C.: The emergence of isolated coherent vortices in turbulent flow, J. Fluid Mech., 140, 21-43, 1984.

McWilliams, J. C. and Flierl, G. R.: On the evolution of isolated, nonlinear vortices, J. Phys. Oceanogr., 9, 1155-1182, 1979.

Moffat, H. K.: The degree of knottendness of tangled vortex lines, J. Fluid Mech., 35, 117-129, 1969.

Molinari, J.: Paradigms of tropical cyclogenesis, B. Am. Meteorol. Soc., 85, 662-663, 2004.

Molinari, J., Skubis, S., and Vollaro, D.: External influences on hurricane intensity. Part III: potential vorticity structure, J. Atmos. Sci., 52, 3593-3606, 1995.

Molinari, J., Knight, D., Dickinson, M., Vollaro, D., and Skubis, S.: Potential vorticity, easterly waves, and eastern Pacific tropical cyclogenesis, Mon. Weather Rev., 125, 2699-2708, 1997.

Molinari, J., Vollaro, D., Skubis, S., and Dickinson, M.: Origins and mechanisms of eastern Pacific tropical cyclogenesis: a case study, Mon. Weather Rev., 128, 125-139, 2000.

Molinari, J. and Vollaro, D.: Planetary- and synoptic-scale influences on eastern Pacific tropical cyclogenesis, Mon. Weather Rev., 128, 3296-3307, 2000.

Molinari, J., Vollaro, D., and Corbosiero, K. L.: Tropical cyclone formation in a sheared environment: a case study, J. Atmos. Sci., 61, 2493-2509, 2004.

Molinari, J., Dodge, P., Vollaro, D., Corbosiero, K. L., and Marks, F.: Mesoscale aspects of the downshear reformation of a tropical cyclone, J. Atmos. Sci., 63, 341-354, 2006.

Molinari, J., Lombardo, K., and Vollaro, D.: Tropical cyclogenesis within an equatorial Rossby wave packet, J. Atmos. Sci., 64, 1301-1317, 2007.

Montgomery, M. T. and Farrell, B. F.: Tropical cyclone formation, J. Atmos. Sci., 50, 285-310, 1993.

Montgomery, M. T. and Kallenbach, R. J.: A theory for vortex Rossby waves and its application to spiral bands and intensity changes in hurricanes, Q. J. Roy. Meteorol. Soc., 123, 435-465, 1997.
Montgomery, M. T., Moller, J. D., and Nicklas, C. T.: Linear and nonlinear vortex motion in an asymmetric balance shallow water model, J. Atmos. Sci., 56, 749-768, 1999.

Montgomery, M. T., Bell, M. M., Aberson, S. D., and Black, M. L.: Hurricane Isabel (2003): new insights into the physics of intense storms. Part I: mean vortex structure and maximum intensity estimates, B. Am. Meteorol. Soc., 87, 1335-1347, 2006 a.

Montgomery, M. T., Nicholls, M. E., Cram, T. A., and Saunders, A. B.: A vortical hot tower route to tropical cyclogenesis, J. Atmos. Sci., 63, 355-386, 2006b.

Montgomery, M. T., Nguyen, V. S., and Smith, R. K.: Do tropical cyclones intensify by WISHE?, Q. J. Roy. Meteorol. Soc., in press, 2009.

Montgomery, M. T., Wang, Z., and Dunkerton, T. J.: Intermediate and high-resolution simulations of the transition of a tropical wave critical layer to a tropical depression, Atmos. Chem. Phys. Discuss., in review, 2009.

Montgomery, M. T.: Interactions of tropical waves and diabatic vortices in tropical cyclone formation, NASA-TCSP/NAMMA workshop, Invited plenary presentation, Baltimore, Maryland, May 2007.

Moore, R. W. and Montgomery, M. T.: Re-examining the dynamics of short-scale, diabatic Rossby waves and their role in midlatitude moist cyclogenesis, J. Atmos. Sci., 61, 754-768, 2004.

Moore, R. W. and Montgomery, M. T.: An idealized threedimensional analysis of the diabatic Rossby vortex: A coherent structure of the moist baroclinic atmosphere, J. Atmos. Sci., 62, 2703-2725, 2005.

Moore, R. W. and Montgomery, M. T.: Dynamics of the 24-25 February 2005 cyclone, 13th Cyclone Workshop, Pacific Grove CA, 2006.

Moulin, F. Y. and Flor, J. B.: Experimental study on wave breaking and mixing properties in the periphery of an intense vortex, Dyn. Atmos. Oceans, 40, 115-130, 2005.

Nolan, D. S., Rappin, E. D., and Emanuel, K. A.: Could hurricanes form from random convection in a warmer world?, AMS 27th Conference on Hurricanes and Tropical Meteorology, 2006.

Ooyama, K.: Numerical simulation of the life cycle of tropical cyclones, J. Atmos. Sci., 26, 3-40, 1969.

Panetta, R. L.: Zonal jets in wide baroclinically unstable regions: persistence and scale selection, J. Atmos. Sci., 50, 2073-2106, 1993.

Pierrehumbert, R.: Local and global baroclinic instability of zonally varying flow, J. Atmos. Sci., 41, 2141-2162, 1984.

Pozrikidis, C.: Introduction to Theoretical and Computational Fluid Dynamics, Oxford University Press, 675 pp, 1997.

Randel, W. J.: Upper tropospheric equatorial waves in ECMWF analyses, Q. J. Roy. Meteorol. Soc., 118, 365-394, 1992.

Raymond, D. J., López-Carrillo, C., and López Cavazos, L.: Casestudies of developing east Pacific easterly waves, Q. J. Roy. Meteorol. Soc., 124, 2005-2034, 1998.

Raymond, D. J., Bretherton, C. S., and Molinari, J.: Dynamics of the Intertropical Convergence Zone of the East Pacific, J. Atmos. Sci., 63, 582-597, 2006.

Raymond, D. J. and Sessions, S. L.: Evolution of convection during tropical cyclogenesis, Geophys. Res. Lett., 34, L06811, doi:10.1029/2006GL028607, 2007.

Reasor, P. D. and Montgomery, M. T.: Three dimensional alignment and corotation of weak, TC-like vortices via linear vortex Rossby 
waves, J. Atmos. Sci., 58, 2306-2330, 2001.

Reasor, P. D., Montgomery, M. T., and Grasso, L. D.: A new look at the problem of tropical cyclones in vertical shear flow: vortex resiliency, J. Atmos. Sci., 61, 3-22, 2004.

Reed, R. J., Norquist, D. C., and Recker, E. E.: The structure and properties of African wave disturbances as observed during phase III of GATE, Mon. Weather Rev., 105, 317-333, 1977.

Ritchie, E. A. and Holland, G. J.: Scale interactions during the formation of Typhoon Irving, Mon. Weather Rev., 125, 1377-1396, 1997.

Ritchie, E. A. and Holland, G. J.: Large-scale patterns associated with tropical cyclogenesis in the western Pacific, Mon. Weather Rev., 127, 2027-2043, 1999.

Ritchie, E. A., Simpson, J., Liu, W. T., Halverson, J., Velden, C., Brueske, K. F., and Pierce, H.: Present day satellite technology for hurricane research: a closer look at formation and intensification, Hurricane! Coping with disaster: progress and challenges since Galveston, 1900, AGU monograph, 359 p., 2003.

Rotunno, R. and Emanuel, K. A.: An air-sea interaction theory for tropical cyclones. Part II: Evolutionary study using a nonhydrostatic axisymmetric numerical model, J. Atmos. Sci., 44, 542561, 1987.

Rozoff, C. M., Schubert, W. H., McNoldy, B. D., and Kossin, J. P.: Rapid filamentation zones in intense tropical cyclones, J. Atmos. Sci., 63, 325-340, 2006.

Schecter, D. A., Montgomery, M. T., and Reasor, P. D.: A theory for the vertical alignment of a quasi-geostrophic vortex, J. Atmos. Sci., 59, 150-168, 2002.

Schecter, D. A. and Dunkerton, T. J.: Hurricane formation in diabatic Ekman turbulence, Q. J. Roy. Meteorol. Soc., 135(641), 823-838, 2009

Schubert, W. H. and Hack, J. J.: Transformed Eliassen balanced vortex model, J. Atmos. Sci., 40, 1571-1583, 1983.

Schubert, W. H., Ciesielski, P. E., Stevens, D. E., and Kuo, H.-C.: Potential vorticity modeling of the ITCZ and the Hadley circulation, J. Atmos. Sci., 48, 1493-1509, 1991.

Schumacher, A. B., DeMaria, M., and Knaff, J. A.: Objective estimation of the 24-h probability of tropical cyclone formation, Wea. Forecasting, 24, 456-471, 2009.

Shapiro, L. J.: Tropical storm formation from easterly waves: a criterion for development, J. Atmos. Sci., 34, 1007-1022, 1977.

Shapiro, L. J.: The effect of nonlinearities on the evolution of barotropic easterly waves in a nonuniform environment, J. Atmos. Sci., 37, 2631-2643, 1980.

Shapiro, L. J.: The asymmetric boundary layer flow under a translating hurricane, J. Atmos. Sci., 40, 1984-1998, 1983.

Shapiro, L. J. and Willoughby, H. E.: The response of balanced hurricanes to local sources of heat and momentum, J. Atmos. Sci., 39, 378-394, 1982.

Sharp, R. J., Bourassa, M. A., and O'Brien, J. J.: Early detection of tropical cyclones using Seawinds-derived vorticity, B. Am. Meteorol. Soc., 83, 879-889, 2002.

Simpson, R. H., Frank, N., Shideler, D., and Johnson, H. M.: Atlantic tropical disturbances, 1967, Mon. Weather Rev., 96, 251259, 1968

Simpson, J., Ritchie, E., Holland, G. J., Halverson, J., and Stewart, S.: Mesoscale interactions in tropical cyclone genesis, Mon. Weather Rev., 125, 2643-2661, 1997.

Smith, R. K.: The surface boundary layer of a hurricane, Tellus, 20,
473-484, 1968.

Smith, R. K., Montgomery, M. T., and Vogl, S.: A critique of Emanuel's hurricane model and potential intensity theory, Q. J. Roy. Meteorol. Soc., 134, 551-561, 2008.

Sobel, A. H. and Bretherton, C. S.: Development of synoptic-scale disturbances over the summertime tropical northwest Pacific, J. Atmos. Sci., 56, 3106-3127, 1999.

Sobel, A. H., Nilsson, J., and Polvani, L. M.: The weak temperature gradient approximation and balanced tropical moisture waves, J Atmos. Sci., 58, 3650-3665, 2001.

Takayabu, Y. N. and Nitta, T.: 3-5 day-period disturbances coupled with convection over the tropical Pacific Ocean, J. Meteorol. Soc. Japan, 71(2), 221-246, 1993.

Thorncroft, C. D. and Hoskins, B. J.: An idealized study of African easterly waves. I: a linear view, Q. J. Roy. Meteorol. Soc., 120, 953-982, 1994a.

Thorncroft, C. D. and Hodges, K.: African easterly wave variability and its relationship to Atlantic tropical cyclone activity, J. Climate, 14, 1166-1179, 2001.

Thorncroft, C. D., Parker, D. J., Burton, R. R., et al.: The JET2000 project: aircraft observations of the African easterly jet and African easterly waves, B. Am. Meteorol. Soc., 84, 337-351, 2003.

Tory, K. J. and Montgomery, M. T.: Internal influences on tropical cyclone formation. Topic 2.2 in: Sixth International Workshop on Tropical Cyclones, San Jose, Costa Rica, World Meteorological Organization, 22 pp, 2006.

Tory, K. J., Montgomery, M. T., and Davidson, N. E.: Prediction and diagnosis of tropical cyclone formation in an NWP system. Part I: the critical role of vortex enhancement in deep convection, J. Atmos. Sci., 63, 3077-3090, 2006 a.

Tory, K. J., Montgomery, M. T., Davidson, N. E., and Kepert, J. E.: Prediction and diagnosis of tropical cyclone formation in a NWP system. Part II: a diagnosis of tropical cyclone Chris formation, J. Atmos. Sci., 63, 3091-3113, 2006 b.

Tory, K. J., Davidson, N. E., and Montgomery, M. T.: Prediction and diagnosis of tropical cyclone formation in an NWP system. Part III: diagnosis of developing and non-developing storms, J. Atmos. Sci., 64, 3195-3213, 2007.

Tuleya, R. E. and Kurihara, Y.: A numerical study on the effects of environmental flow on tropical storm genesis, Mon. Weather Rev., 109, 2487-2506, 1981.

Van Sang, N., Smith, R., and Montgomery, M. T.: Tropical cyclone intensification and predictability in three dimensions, Q. J. Roy. Meteorol. Soc., 134, 563-582, 2008.

Vecchi, G. A. and Soden, B. J.: Increased tropical Atlantic wind shear in model projections of global warming, Geophys. Res. Lett., 34, L08702, doi:10.1029/2006GL028905, 2007.

Velden, C. S., Hayden, C. M., Nieman, S. J., Menzel, W. P., Wanzong, S., and Goerss, J. S.: Upper-tropospheric winds derived from geostationary satellite water vapor observations, B. Am. Meteorol. Soc., 78, 173-195, 1997.

Velden, C., Daniels, J., Stettner, D., et al.: Recent innovations in deriving tropospheric winds from meteorological satellites, B. Am. Meteorol. Soc., 86, 205-223, 2005.

Ventham, J. D. and Wang, B.: Large-scale flow patterns and their influence on the intensification rates of Western North Pacific tropical storms, Mon. Weather Rev., 135, 1110-1127, 2007.

Vimont, D. J. and Kossin, J. P.: The Atlantic Meridional 
Mode and hurricane activity Geophys. Res. Lett., 34, L07709, doi:10.1029/2007GL029683, 2007.

Wang, C.-C. and Magnusdottir, G.: ITCZ breakdown in threedimensional flows, J. Atmos. Sci., 62, 1497-1512, 2005.

Wang, C.-C. and Magnusdottir, G.: The ITCZ in the central and eastern Pacific on synoptic time scales, Mon. Weather Rev., 134, 1405-1421, 2006.

Webster, P. J. and Holton, J. R.: Cross-equatorial response to middle-latitude forcing in a zonally varying basic state, J. Atmos. Sci., 39, 722-733, 1982.

Weisman, M. L. and Rotunno, R.: The use of vertical wind shear versus helicity in interpreting supercell dynamics, J. Atmos. Sci., 57, 1452-1472, 2000.

Wernli, H. and Kenzelmann, P.: Diabatic Rossby waves: Aspects of their dynamics, climatology and predictability, 13th Cyclone Workshop, Pacific Grove CA, 2006.

Wheeler, M. and Kiladis, G. N.: Convectively coupled equatorial waves: analysis of clouds and temperature in the wavenumberfrequency domain, J. Atmos. Sci., 56, 374-399, 1999.

Wheeler, M., Kiladis, G. N., and Webster, P. J.: Large-scale dynamical fields associated with convectively coupled equatorial waves, J. Atmos. Sci., 57, 613-640, 2000.
Wilson, J. D. and Mak, M.: Tropical response to lateral forcing with a latitudinally and zonally nonuniform basic state, J. Atmos. Sci., 41, 1187-1201, 1984.

Wing, A. A., Sobel, A. H., and Camargo, S. J.: Relationship between the potential and actual intensities of tropical cyclones on interannual time scales, Geophys. Res. Lett., 34, L08810, doi:10.1029/2006GL028581, 2007.

Wirth, V. and Dunkerton, T. J.: A unified perspective on the dynamics of axisymmetric hurricanes and monsoons, J. Atmos. Sci., 63, 2529-2547, 2006.

Wong, S. and Dessler, A. E.: Suppression of deep convection over the tropical North Atlantic by the Saharan Air Layer, Geophys. Res. Lett., 32, L09808, doi:10.1029/2004GL022295, 2005.

Zehnder, J. A., Powell, D. M., and Ropp, D. L.: The interaction of easterly waves, orography, and the Intertropical Convergence Zone in the genesis of eastern Pacific tropical cyclones, Mon. Weather Rev., 127, 1566-1585, 1999.

Zhang, C. and Webster, P. J.: Laterally forced equatorial perturbations in a linear model. Part I: stationary transient forcing, J. Atmos. Sci., 49, 585-607, 1992.

Zhu, Y. and Newell, R. E.: A proposed algorithm for moisture fluxes from atmospheric rivers, Mon. Weather Rev., 126, 725735, 1998. 